\title{
DOUBLE AFFINE HECKE ALGEBRAS AND BISPECTRAL QUANTUM KNIZHNIK-ZAMOLODCHIKOV EQUATIONS
}

\author{
MICHEL VAN MEER AND JASPER V. STOKMAN
}

\begin{abstract}
We use the double affine Hecke algebra of type $\mathrm{GL}_{N}$ to construct an explicit consistent system of $q$-difference equations, which we call the bispectral quantum Knizhnik-Zamolodchikov (BqKZ) equations. BqKZ includes, besides Cherednik's quantum affine $\mathrm{KZ}$ equations associated to principal series representations of the underlying affine Hecke algebra, a compatible system of $q$-difference equations acting on the central character of the principal series representations. We construct a meromorphic self-dual solution $\Phi$ of $\mathrm{BqKZ}$ which, upon suitable specializations of the central character, reduces to symmetric self-dual Laurent polynomial solutions of quantum $\mathrm{KZ}$ equations. We give an explicit correspondence between solutions of BqKZ and solutions of a particular bispectral problem for Ruijsenaars' commuting trigonometric $q$ difference operators. Under this correspondence $\Phi$ becomes a self-dual HarishChandra series solution $\Phi^{+}$of the bispectral problem. Specializing the central character as above, we recover from $\Phi^{+}$the symmetric self-dual Macdonald polynomials.
\end{abstract}

\section{Contents}

1. Introduction

2. The double affine Hecke algebra

3. The bispectral quantum KZ equations

4. The explicit form of the bispectral quantum KZ equations 12

5. Solutions of the bispectral quantum KZ equations 19

6. The correspondence with bispectral problems 28

7. Polynomial theory 38

8. Appendix on holonomic systems of $q$-difference equations 45

References 51

\section{INTRODUCTION}

Let $M$ be an $N$ !-dimensional complex vector space and write $T=(\mathbb{C} \backslash\{0\})^{N}$. We derive an explicit holonomic system of $q$-difference equations on $M$-valued meromorphic functions on $T \times T$, which we call the bispectral quantum KnizhnikZamolodchikov (BqKZ) equations. It is related to Cherednik's 9] quantum affine $\mathrm{KZ}$ equations in the following way.

2000 Mathematics Subject Classification. 33D80, 33D52.

Key words and phrases. Double affine Hecke algebras, quantum Knizhnik-Zamolodchikov equations, Macdonald polynomials, bispectral problems. 
Let $H$ be the extended affine Hecke algebra of type $\mathrm{GL}_{N}$. For fixed $\zeta \in T$ the vector space $M$ admits an $H$-module structure, turning $M$ into the principal series module $M(\zeta)$ of $H$ with central character $\zeta$. The BqKZ naturally splits up into two subsystems of $q$-difference equations. The first subsystem acts only on the first $T$-component of $T \times T$ and as such it realizes, for any fixed $\zeta \in T$, Cherednik's [9] quantum affine $\mathrm{KZ}$ equation $\mathrm{qKZ}_{\zeta}$ acting on $M(\zeta)$-valued meromorphic functions on $T \times\{\zeta\}$. The second, dual subsystem is obtained from the first by replacing the role of $(t, \zeta) \in T \times T$ by $\left(\zeta^{-1}, t^{-1}\right)$ and conjugating the $q$-connection matrices by an explicit complex linear automorphism $C_{\iota}$ of $M$. Hence, it acts only on the second $T$-component of $T \times T$ and it essentially realizes $\mathrm{qKZ}_{t^{-1}}$ for fixed $t \in T$. In particular, this provides a quantum isomonodromic interpretation of qKZ. This should be compared with the interpretation of rational KZ equations as quantizations of Schlesinger equations, see [38] and [22].

The BqKZ is constructed using Cherednik's [9] double affine Hecke algebra $\mathbb{H}$ of type $\mathrm{GL}_{N}$. As a vector space $\mathbb{H}$ is isomorphic to $\mathbb{C}[T] \otimes H \simeq \mathbb{C}[T] \otimes H_{0} \otimes \mathbb{C}[T] \simeq$ $\mathbb{C}[T \times T] \otimes H_{0}$ with $H_{0}$ the finite Hecke algebra of type $A_{N-1}$. Cherednik's antialgebra involution $*: \mathbb{H} \rightarrow \mathbb{H}$ essentially interchanges, under the above vector space identification, the role of the two copies of $\mathbb{C}[T]$. For $w, w^{\prime} \in W=S_{N} \ltimes \mathbb{Z}^{N}$ we consider the map $h \mapsto \widetilde{S}_{w} h \widetilde{S}_{w^{\prime}}^{*}(h \in \mathbb{H})$, where the $\widetilde{S}_{w} \in \mathbb{H}$ are Cherednik's nonnormalized (X-)intertwiners. Restricted to $w, w^{\prime} \in \mathbb{Z}^{N}$, suitable renormalizations of these maps become the $q$-connection matrices of $\mathrm{BqKZ}$, with $H_{0}$ playing the role of $M$. The anti-involution $*$ of $\mathbb{H}$ gives rise to the automorphism $C_{\iota}$ interchanging the $\mathrm{qKZ}$ subsystem of $\mathrm{BqKZ}$ with its dual subsystem in BqKZ.

$\mathrm{qKZ}_{\zeta}$ is gauge equivalent to Frenkel and Reshetikhin's [20] quantum KZ equations associated with the $N$-fold tensor product of the vector representation of quantum $\mathfrak{s l}_{N}$ (see [9, §1.3.2]). A special case of $\mathrm{qKZ}_{\zeta}$ was considered earlier by Smirnov 42. Etingof and Varchenko [18] used quantum group methods to construct systems of $q$-difference equations (so-called dynamical $q$-difference equations) that are compatible with Frenkel and Reshetikhin's quantum KZ equations associated to evaluation representations of quantum affine algebras. It is likely that the system of dynamical $q$-difference equations associated with $\mathrm{qKZ}_{\zeta}$ is equivalent to the dual qKZ subsystem in BqKZ.

Preceding the above mentioned work [18] of Etingof and Varchenko, dynamical equations for various degenerations of quantum $\mathrm{KZ}$ equations have been analyzed in detail; see, e.g., [19], 47], 44], [46], 45] and [29]. An interesting aspect in, e.g., 47] and 46], is the observation that various degenerations of quantum KZ equations are the duals of their associated dynamical equations with respect to $\left(\mathfrak{g l}_{r}, \mathfrak{g l}_{s}\right)$ duality. In the present set-up (which corresponds to $r=s=N$ ), this duality is incorporated by the automorphism $C_{\iota}$, which reflects Cherednik's duality anti-involution of the double affine Hecke algebra $\mathbb{H}$ on the level of BqKZ.

We investigate the space SOL of $M$-valued meromorphic solutions of $\mathrm{BqKZ}$ in detail. We first analyze $\mathrm{BqKZ}$ in a suitable asymptotic region. It leads to a solution $\Phi$ of BqKZ which is self-dual, in the sense that $\Phi(t, \gamma)=C_{\iota} \Phi\left(\gamma^{-1}, t^{-1}\right)$ as $M$-valued meromorphic functions in $(t, \gamma) \in T \times T$. We construct a basis of solutions of SOL in terms of $\Phi$, and we give an explicit formula for the leading term of $\Phi(t, \gamma)$ as function of $t$.

Cherednik [6, Thm. 3.4] constructed for arbitrary root systems a correspondence between solutions of quantum KZ equations and solutions of a system of $q$-difference 
equations. In [6. Thm. 4.4], Cherednik made the correspondence precise for $\mathrm{GL}_{N}$. It yields an explicit map $\chi_{+}$from solutions of $\mathrm{qKZ}_{\zeta}$ to solutions of the spectral problem of Ruijsenaars' 39] commuting trigonometric $q$-difference operators with spectral parameter $\zeta^{-1}$ (the Ruijsenaars operators are also frequently referred to as Macdonald-Ruijsenaars operators). The latter result has been generalized to arbitrary root systems in [26, Thm. 4.6] and [7. We analyze the map $\chi_{+}$in the present bispectral setting. It leads to the interpretation of $\chi_{+}$as an embedding of SOL into the space of meromorphic solutions of a bispectral problem involving the above Ruijsenaars operators as well as Ruijsenaars operators acting on the spectral parameter.

The application of the correspondence to the self-dual solution $\Phi$ of BqZK leads to a self-dual Harish-Chandra series solution $\Phi^{+}$of the bispectral problem. HarishChandra series solutions of the Ruijsenaars operators with fixed spectral parameter were investigated before in, e.g., [15, [16, 27] and 31. The present approach to Harish-Chandra series, which uses quantum KZ equations in an essential way, has the advantage that it leads to new results on the convergence and singularities of the Harish-Chandra series. These results, together with Cherednik's recent work [10, form important building blocks in deriving the $c$-function expansion of Cherednik's global $(q, t)$-spherical function (this will be detailed in a forthcoming paper of the second author).

The $q$-connection matrices of the dual qKZ subsystem of BqKZ can be used to map solutions of $\mathrm{qKZ}_{\zeta}$ for a fixed central character $\zeta \in T$ to solutions of $\mathrm{qKZ}_{\zeta^{\prime}}$ with respect to a suitably shifted central character $\zeta^{\prime}$. Applied to a constant solution we obtain a symmetric Laurent polynomial solution $Q_{\lambda}$ for any non-increasing $N$ tuple $\lambda$ of integers, which is automatically self-dual. We express $Q_{\lambda}$ in terms of a suitable specialization of the solution $\Phi$ of BqKZ. Applying $\chi_{+}$to $Q_{\lambda}$ we obtain a symmetric self-dual Laurent polynomial eigenfunction of the Ruijsenaars operators, which is the normalized Macdonald [32] polynomial of degree $\lambda$. We show that the well-known duality and evaluation formula for the Macdonald polynomials (see 32, Chpt. VI]) are direct consequences of the properties of the Laurent polynomials $Q_{\lambda}$.

For various other approaches to the construction of solutions of quantum KZ equations see, e.g., [20], [14], 24], [27], [36], [11] and [25].

Many results in the present paper, in particular the BqZK equations themselves, can be extended to arbitrary root systems. These extensions use the root system generalizations of $\mathbb{H}$ and their fundamental properties (see [9]). We have chosen to restrict the present exposition to the $\mathrm{GL}_{N}$ theory, not only in order to present the basic ideas while limiting the technicalities, but also because the $\mathrm{GL}_{N}$ theory deserves extra attention by its particularly strong ties with quantum groups and quantum integrable lattice models (see, e.g., 14, 24]). The theory for arbitrary root systems is detailed in a forthcoming paper of the first author.

\section{Conventions}

- $\otimes$ always stands for tensor product over $\mathbb{C}$ and $\operatorname{End}(M)$, for a module $M$ over $\mathbb{C}$, stands for $\mathbb{C}$-linear endomorphisms.

$-\mathbb{N}=\{1,2, \ldots\}$.

- For a module $M$ over a commutative ring $R$ and a ring extension $R \subset S$, we write $M^{S}=S \otimes_{R} M$. 


\section{Acknowledgments}

Both authors are supported by the Netherlands Organization for Scientific Research (NWO) in the VIDI-project "Symmetry and modularity in exactly solvable models". We thank the referee for valuable comments.

\section{The double affine Hecke algebra}

2.1. The extended affine Weyl group. Let $N \geq 2$ and let $D=D_{N}$ be the affine Dynkin diagram of affine type $\widehat{A}_{N-1}$ (the cyclic graph with $N$ vertices if $N \geq 3$ ). The $N$ vertices are labeled by the numbers $0,1, \ldots, N-1$ (anticlockwise if $N \geq 3$ ). We identify occasionally the set of labels by the group $\mathbb{Z}_{N}$ of integers modulo $N$.

Write $W_{Q}$ for the affine Weyl group of affine type $\widehat{A}_{N-1}$. In terms of its Coxeter generators $s_{i}\left(i \in \mathbb{Z}_{N}\right)$, the characterizing group relations are the quadratic relations $s_{i}^{2}=1$ and, if $N \geq 3$, the braid relations

$$
\begin{aligned}
s_{i} s_{i+1} s_{i} & =s_{i+1} s_{i} s_{i+1}, \\
s_{i} s_{j} & =s_{j} s_{i}, \quad i-j \neq 0, \pm 1 .
\end{aligned}
$$

The subgroup generated by $s_{1}, \ldots, s_{N-1}$ is isomorphic to the symmetric group $S_{N}$ in $N$ letters, where $s_{i}$ is identified with the simple transposition $i \leftrightarrow i+1$.

Let $\operatorname{Aut}(D)$ be the group of automorphisms of the affine Dynkin diagram of type $\widehat{A}_{N-1}$. Let $c \in \operatorname{Aut}(D)$ the element of order $N$, acting on the label set $\mathbb{Z}_{N}$ of the vertices of $D$ by $c(i)=i+1$. We view $c$ as automorphism of $W_{Q}$ by $c\left(s_{i}\right)=s_{i+1}$.

Let $\Omega=\langle\pi\rangle$ be the infinite cyclic group with cyclic generator $\pi$. It acts by group automorphisms on $W_{Q}$ by $\pi \mapsto c$. Accordingly we can define the semi-direct product group $W=\Omega \ltimes W_{Q}$, which is called the extended affine Weyl group (associated to $\left.\mathrm{GL}_{N}\right)$. We denote $e$ for the identity element of $W$.

Since $s_{0}=\pi s_{N-1} \pi^{-1}$, the subgroups $S_{N}$ and $\Omega$ already generate $W$ as a group. Furthermore we have $W \simeq S_{N} \ltimes \mathbb{Z}^{N}$. The cyclic generator $\pi$ of $\Omega$ corresponds to $\pi=$ $\sigma \epsilon_{N}$, where $\left\{\epsilon_{i}\right\}_{i=1}^{N}$ denotes the standard $\mathbb{Z}$-basis of $\mathbb{Z}^{N}$ and $\sigma=s_{1} s_{2} \cdots s_{N-1} \in S_{N}$ is the "clockwise rotation" which maps $N$ to 1 and all other $i$ to $i+1$. Conversely,

$$
\epsilon_{j}=s_{j-1} \cdots s_{2} s_{1} \pi s_{N-1} s_{N-2} \cdots s_{j}
$$

for $j=1, \ldots, N$.

Remark 2.1. Under the identification $W \simeq S_{N} \ltimes \mathbb{Z}^{N}$, we have $W_{Q}=S_{N} \ltimes Q$ with $Q \subset \mathbb{Z}^{N}$ the sublattice of rank $N-1$ consisting of $N$-tuples of integers that sum up to zero (this is the (co)root lattice of the root system $R=\left\{\epsilon_{i}-\epsilon_{j}\right\}_{1 \leq i \neq j \leq N}$ of type $\left.A_{N-1}\right)$.

For $w \in W$ let $w^{\prime} \in W_{Q}$ and $\omega \in \Omega$ be the unique group elements such that $w=w^{\prime} \omega$, then we define the length $\ell(w)$ of $w$ to be the length of $w^{\prime} \in W_{Q}$, i.e., it is the minimal number $r$ such that $w^{\prime}$ can be expressed as

$$
w^{\prime}=s_{i_{1}} \cdots s_{i_{r}}
$$

for some $i_{k} \in \mathbb{Z}_{N}$ (such an expression of $w^{\prime}$, as well as the resulting expression for $w=w^{\prime} \omega$, is called a reduced expression). Thus $\Omega$ consists of the elements of $W$ of length zero.

A central role in this paper is played by an action of the extended affine Weyl group $W$ by $q$-difference reflection operators on suitable function spaces on $T:=$ $\left(\mathbb{C}^{\times}\right)^{N}$, where $\mathbb{C}^{\times}:=\mathbb{C} \backslash\{0\}$. Here $q$ is taken to be real and strictly between zero and one (with minor technical adjustments the condition on $q$ may be relaxed to 
$0<|q|<1$, and a parallel theory can be developed for $|q|>1$ ). Since $q$ is fixed once and for all, we will in general suppress the dependence on $q$ in notations. We start with an action of $W$ on $T$ by

$$
\begin{aligned}
w t & =\left(t_{w^{-1}(1)}, \ldots, t_{w^{-1}(N)}\right), \quad w \in S_{N}, \\
\lambda t & =\left(q^{\lambda_{1}} t_{1}, \ldots, q^{\lambda_{N}} t_{N}\right), \quad \lambda=\left(\lambda_{1}, \ldots, \lambda_{N}\right) \in \mathbb{Z}^{N},
\end{aligned}
$$

for $t=\left(t_{1}, \ldots, t_{N}\right) \in T$. It is convenient to introduce $\kappa^{\lambda}:=\left(\kappa^{\lambda_{1}}, \ldots, \kappa^{\lambda_{N}}\right)$ for $\kappa \in \mathbb{C}^{\times}$and $\lambda \in \mathbb{Z}^{N}$, so that the action of $\lambda \in \mathbb{Z}^{N}$ on $t \in T$ can simply be written as

$$
\lambda t=q^{\lambda} t
$$

in standard vector notation. Note that the action of $\pi \in \Omega$ is given by

$$
\pi\left(t_{1}, \ldots, t_{N}\right)=\left(q t_{N}, t_{1}, \ldots, t_{N-1}\right) .
$$

Consider the algebra $\mathbb{C}[T]=\mathbb{C}\left[x_{1}^{ \pm 1}, \ldots, x_{N}^{ \pm 1}\right]$ of complex-valued, regular functions on $T$, where $x_{i}(t):=t_{i}$ for $t=\left(t_{1}, \ldots, t_{N}\right) \in T$ are the standard coordinate functions. We write

$$
x^{\lambda}:=x_{1}^{\lambda_{1}} \cdots x_{N}^{\lambda_{N}} \in \mathbb{C}[T]
$$

for $\lambda=\left(\lambda_{1}, \ldots, \lambda_{N}\right) \in \mathbb{Z}^{N}$, which form the monomial basis of $\mathbb{C}[T]$.

Let $\mathbb{C}(T)$ be the field of rational functions on $T, \mathcal{O}(T)$ be the ring of analytic functions on $T$, and $\mathcal{M}(T)$ be the field of meromorphic functions on $T$. Note that $\mathcal{M}(T)$ is the quotient field of $\mathcal{O}(T)$ (cf. 23, Thm. 7.4.6]). The $W$-action on $T$ gives rise to a left $W$-action by algebra automorphisms on $\mathbb{C}[T], \mathbb{C}(T), \mathcal{O}(T)$ and $\mathcal{M}(T)$, via

$$
(w f)(t)=f\left(w^{-1} t\right)
$$

for $w \in W, t \in T$. We can, in particular, form the smashed product algebra $\mathbb{C}(T) \# W$. Recall that if $G$ is a group and $A$ is a $G$-algebra over $\mathbb{C}$ (that is, a unital associative algebra over $\mathbb{C}$ endowed with a left $G$-action by algebra automorphisms), then the smashed product algebra $A \# G$ is the unique complex unital associative algebra such that

(i) $A \# G=A \otimes \mathbb{C}[G]$ as a complex vector space;

(ii) the canonical linear embeddings $A \hookrightarrow A \# G, \mathbb{C}[G] \hookrightarrow A \# G$ are algebra homomorphisms; and

(iii) the cross relations

$$
(a \otimes g) \cdot(b \otimes h)=a g(b) \otimes g h,
$$

are satisfied for $a, b \in A$ and $g, h \in G$.

We will always write $a g:=a \otimes g \in A \# G(a \in A, g \in G)$. Observe that $A \# G$ canonically acts on any $G$-algebra $B$ containing $A$ as a $G$-subalgebra.

Note that the smashed product algebra $\mathbb{C}(T) \# W$ depends on $q$, since the $W$ action on $\mathbb{C}(T)$ depends on $q$ (see (2.3) $)$. Sometimes it is convenient to emphasize its $q$-dependence, in which case we write $\mathbb{C}(T) \#_{q} W$ instead of $\mathbb{C}(T) \# W$.

The canonical left $\mathbb{C}(T) \# W$-action on $\mathbb{C}(T)$ (and $\mathcal{M}(T)$ ) is faithful and realizes $\mathbb{C}(T) \# W$ as the algebra of $q$-difference $S_{N}$-reflection operators with coefficients in $\mathbb{C}(T)$. If $f \in \mathbb{C}(T)$ then we write $f(X)$ for the associated element in $\mathbb{C}(T) \# W$ (it is the operator defined as multiplication by $f$ ). In particular, $X_{i}$ is multiplication by the coordinate function $x_{i}$. 
2.2. The extended affine Hecke algebra and Cherednik's basic representation. In this subsection we recall some constructions and results due to Cherednik (see, e.g., [9, Chpt. 1] and references therein).

Fix a nonzero complex number $k$.

Definition 2.2. The affine Hecke algebra $H_{Q}=H_{Q}(k)$ is the complex, unital, associative algebra generated by $T_{i}\left(i \in \mathbb{Z}_{N}\right)$ and satisfying

(1) if $N \geq 3$, the braid relations

$$
\begin{aligned}
T_{i} T_{i+1} T_{i} & =T_{i+1} T_{i} T_{i+1}, \\
T_{i} T_{j} & =T_{j} T_{i}, \quad i-j \neq 0, \pm 1 ;
\end{aligned}
$$

(2) the quadratic relations $\left(T_{i}-k\right)\left(T_{i}+k^{-1}\right)=0$.

The Dynkin diagram automorphism $c \in \operatorname{Aut}(D)$ can also be viewed as automorphism of $H_{Q}$ by $c\left(T_{i}\right)=T_{i+1}$. Accordingly, $\Omega$ acts by algebra automorphisms on $H_{Q}$ by $\pi \mapsto c$. The extended affine Hecke algebra $H=H(k)$ is the associated smashed product algebra $H_{Q} \# \Omega$.

For a reduced expression $w=s_{i_{1}} \cdots s_{i_{r}} \omega \in W\left(i_{k} \in \mathbb{Z}_{N}, \omega \in \Omega\right)$, the element

$$
T_{w}:=T_{i_{1}} \cdots T_{i_{r}} \omega \in H
$$

is well defined. The $T_{w}(w \in W)$ form a linear basis of $H$. For $k=1$, the extended affine Hecke algebra $H$ is isomorphic to the group algebra $\mathbb{C}[W]$ of $W$ via the identification $T_{w} \leftrightarrow w(w \in W)$.

The finite Hecke algebra is the subalgebra $H_{0}$ of $H$ generated by $T_{1}, \ldots, T_{N-1}$. The elements $T_{w}\left(w \in S_{N}\right)$ form a linear basis of $H_{0}$. Note that $H$ is already generated as algebra by $H_{0}$ and $\pi^{ \pm 1}$, since $T_{0}=\pi T_{N-1} \pi^{-1}$.

Put

$$
Y_{i}:=T_{i-1}^{-1} \cdots T_{2}^{-1} T_{1}^{-1} \pi T_{N-1} T_{N-2} \cdots T_{i} \in H
$$

for $i=1, \ldots, N$. Note that $Y_{i}$ becomes the translation element $\epsilon_{i}$ in $W$ if $k=1$. We furthermore write $Y^{\lambda}:=Y_{1}^{\lambda_{1}} \cdots Y_{N}^{\lambda_{N}}$ for $\lambda=\left(\lambda_{1}, \ldots, \lambda_{N}\right) \in \mathbb{Z}^{N}$. We have the following characterization of $H$, due to Bernstein. For details we refer to Lusztig [30] or Macdonald [34, §4.2].

Theorem 2.3. $H$ is the unique unital complex associative algebra, such that

(i) $H_{0} \otimes \mathbb{C}[T] \simeq H$ as complex vector spaces, via $h \otimes f \mapsto h f(Y)$ for $h \in H_{0}$, $f \in \mathbb{C}[T]$, where $f(Y)=\sum_{\lambda} c_{\lambda} Y^{\lambda}$ if $f=\sum_{\lambda} c_{\lambda} x^{\lambda} \in \mathbb{C}[T]$

(ii) the canonical maps $H_{0}, \mathbb{C}[T] \hookrightarrow H$ are algebra embeddings; we write $\mathbb{C}_{Y}[T]=$ $\operatorname{span}_{\mathbb{C}}\left\{Y^{\lambda}\right\}_{\lambda \in \mathbb{Z}^{N}}$ for the image of $\mathbb{C}[T]$ in $H$; and

(iii) the following cross relations

$$
\begin{aligned}
T_{i}^{-1} Y_{i} T_{i}^{-1} & =Y_{i+1}, \\
Y_{j} T_{i} & =T_{i} Y_{j}, \quad i \neq j-1, j
\end{aligned}
$$

are satisfied for $1 \leq i<N$ and $1 \leq j \leq N$.

Cherednik realized the affine Hecke algebra $H$ inside the algebra $\mathbb{C}(T) \# W$ of $q$-difference reflection operators as follows. 
Theorem 2.4. There is a unique injective algebra homomorphism $\rho=\rho_{k, q}: H(k) \rightarrow$ $\mathbb{C}(T) \#_{q} W$ satisfying

$$
\begin{aligned}
\rho\left(T_{i}\right) & =k+c_{k}\left(X_{i} / X_{i+1}\right)\left(s_{i}-1\right), \\
\rho(\pi) & =\pi,
\end{aligned}
$$

for $i=1, \ldots, N-1$, where

$$
c_{k}(z):=\frac{k^{-1}-k z}{1-z} .
$$

Note that for the affine Hecke algebra $H=H(k)$ with fixed parameter $k$, Theorem 2.4yields a one-parameter family of realizations of $H$ (the additional parameter being $q$ ).

Remark 2.5. The image $\rho(H)$ preserves $\mathbb{C}[T]$, viewed as a subspace of the canonical $\mathbb{C}(T) \# W$-module $\mathbb{C}(T)$. The resulting representation of $H$ on $\mathbb{C}[T]$ is faithful and is called the basic representation of $H$.

We frequently identify $H$ with its image under $\rho$ in $\mathbb{C}(T) \# W$.

We now come to the definition of Cherednik's double affine Hecke algebra which depends, besides on $k$, on the additional parameter $q$.

Definition 2.6. The double affine Hecke algebra $\mathbb{H}=\mathbb{H}(k, q)$ is the subalgebra of $\mathbb{C}(T) \#_{q} W$ generated by $\rho_{k, q}(H)$ and by the multiplication operators $f(X)$ ( $f \in$ $\mathbb{C}[T])$.

Let $\mathbb{L}=\mathbb{C}[T] \otimes \mathbb{C}[T] \simeq \mathbb{C}[T \times T]$ denote the complex-valued regular functions on $T \times T$. We view $\mathbb{H}$ as $\mathbb{L}$-module by

$$
(f \otimes g) \cdot h:=f(X) h g(Y)
$$

for $f, g \in \mathbb{C}[T]$ and $h \in \mathbb{H}$. The following theorem is the so-called Poincaré-BirkhoffWitt (PBW) property of the double affine Hecke algebra.

Theorem 2.7. We have $\mathbb{H} \simeq H_{0}^{\mathbb{L}}=\mathbb{L} \otimes H_{0}$ as $\mathbb{L}$-modules.

The PBW property is an essential ingredient in deriving the characterizing relations for the double affine Hecke algebra $\mathbb{H}$ in terms of its algebraic generators $T_{i}(1 \leq i<N), \pi^{ \pm 1}$ and $X_{j}^{ \pm 1}(1 \leq j \leq N)$. Since we are not going to use this presentation explicitly in this paper, we refer the reader to $[9$ for further details. We use though one of its direct consequences, namely the existence of the duality anti-isomorphism (see Cherednik [9, Thm. 1.4.8]):

Theorem 2.8. There exists a unique $\mathbb{C}$-linear anti-algebra involution $*: \mathbb{H} \rightarrow \mathbb{H}$ determined by

$$
\begin{aligned}
T_{w}^{*} & =T_{w^{-1}}, & & w \in S_{N}, \\
\left(Y^{\lambda}\right)^{*} & =X^{-\lambda}, & & \lambda \in \mathbb{Z}^{N}, \\
\left(X^{\lambda}\right)^{*} & =Y^{-\lambda}, & & \lambda \in \mathbb{Z}^{N} .
\end{aligned}
$$


2.3. Intertwiners. In this subsection we recall the construction of the (nonnormalized) affine intertwiners associated to the double affine Hecke algebra $\mathbb{H}$. The intertwiners play an important role in the construction of a nontrivial $W \times W$ cocycle in the next section. Consider the elements

$$
\begin{aligned}
& \widetilde{S}_{i}=\left(k-k^{-1} X_{i+1} / X_{i}\right) s_{i}, \quad 1 \leq i<N, \\
& \widetilde{S}_{0}=\left(k-k^{-1} q^{-1} X_{1} / X_{N}\right) s_{0}, \\
& \widetilde{S}_{\pi}=\pi
\end{aligned}
$$

in $\mathbb{C}(T) \#_{q} W$. The following facts are well known (cf., e.g., 9, §1.3]). For the convenience of the reader, we give a short sketch of the proof.

Proposition 2.9. Let $w \in W$ and let $w=s_{j_{1}} \cdots s_{j_{r}} \pi^{m}$ be a reduced expression $\left(j_{l} \in \mathbb{Z}_{N}, m \in \mathbb{Z}\right)$.

(i) $\widetilde{S}_{w}:=\widetilde{S}_{j_{1}} \cdots \widetilde{S}_{j_{r}} \widetilde{S}_{\pi}^{m}$ is a well-defined element of $\mathbb{C}(T) \# W$;

(ii) $\widetilde{S}_{w} \in \mathbb{H}$;

(iii) the $\widetilde{S}_{i}\left(i \in \mathbb{Z}_{N}\right)$ satisfy the $\widehat{A}_{N-1}$-type braid relations;

(iv) $\widetilde{S}_{w} f(X)=(w f)(X) \widetilde{S}_{w}$ in $\mathbb{C}(T) \# W$ for all $f \in \mathbb{C}(T)$; and

(v) $\widetilde{S}_{i} \widetilde{S}_{i}=\left(k-k^{-1} X_{i+1} / X_{i}\right)\left(k-k^{-1} X_{i} / X_{i+1}\right)$ for $1 \leq i<N$.

Proof. (i) Set $d_{i}:=\left(k-k^{-1} X_{i+1} / X_{i}\right)(1 \leq i<N)$ and $d_{0}:=\left(k-k^{-1} q^{-1} X_{1} / X_{N}\right)$. We have

$$
\widetilde{S}_{w}=d_{j_{1}}\left(s_{j_{1}} d_{j_{2}}\right) \cdots\left(s_{j_{1}} \cdots s_{j_{r-1}} d_{j_{r}}\right) w
$$

in $\mathbb{C}(T) \# W$. By, e.g., Macdonald [34, (2.2.9)], we know that

$$
d_{w}:=d_{j_{1}}\left(s_{j_{1}} d_{j_{2}}\right) \cdots\left(s_{j_{1}} \cdots s_{j_{r-1}} d_{j_{r}}\right)
$$

is independent of the reduced expression of $w$. Hence $\widetilde{S}_{w} \in \mathbb{C}(T) \# W$ is well defined.

(ii) Note that $\widetilde{S}_{i}$ can be written as

$$
\widetilde{S}_{i}=\left(1-X_{i+1} / X_{i}\right)\left(T_{i}-k\right)+k-k^{-1} X_{i+1} / X_{i}
$$

for $1 \leq i<N$, which shows that it lies in $\mathbb{H}$. Furthermore, $\pi^{ \pm 1} \in \mathbb{H}$, hence $\widetilde{S}_{\pi}^{ \pm 1} \in \mathbb{H}$ and $\widetilde{S}_{0}=\pi \widetilde{S}_{N-1} \pi^{-1} \in \mathbb{H}$. Consequently, $\widetilde{S}_{w} \in \mathbb{H} \subset \mathbb{C}(T) \# W$.

(iii) is immediate from (i), while (iv) and (v) are clear from the definition of the $\widetilde{S}_{i}$ and $\widetilde{S}_{\pi}$.

Definition 2.10. The elements $\widetilde{S}_{w}(w \in W)$ are called the affine intertwiners of $\mathbb{H}$.

\section{The Bispectral quantum KZ EQUations}

3.1. Construction of the cocycle. Let $\iota$ denote the nontrivial element of the two group $\mathbb{Z}_{2}$. We define the group $\mathbb{W}$ as the semidirect product

$$
\mathbb{W}:=\mathbb{Z}_{2} \ltimes(W \times W),
$$

where $\iota \in \mathbb{Z}_{2}$ acts on $W \times W$ by switching the components: $\iota\left(w, w^{\prime}\right)=\left(w^{\prime}, w\right) \iota$ for $w, w^{\prime} \in W$. We first use the double affine Hecke algebra, its affine intertwiners, and its duality anti-isomorphism to construct a group homomorphism $\tau=\tau_{k}: \mathbb{W} \rightarrow$ $\mathrm{GL}_{\mathbb{C}}\left(H_{0}^{\mathbb{K}}\right)$ depending on the Hecke algebra parameter $k$, where $\mathbb{K}:=\mathcal{M}(T \times T)$ is the field of meromorphic functions on $T \times T$. 
The representation $\tau$ will be constructed from the complex linear endomorphisms $\widetilde{\sigma}_{\left(w, w^{\prime}\right)}\left(w, w^{\prime} \in W\right)$ and $\widetilde{\sigma}_{\iota}$ of the double affine Hecke algebra $\mathbb{H}$, defined by

$$
\begin{aligned}
\widetilde{\sigma}_{\left(w, w^{\prime}\right)}(h) & =\widetilde{S}_{w} h \widetilde{S}_{w^{\prime}}^{*}, \\
\widetilde{\sigma}_{\iota}(h) & =h^{*}
\end{aligned}
$$

for $h \in \mathbb{H}$. In the following lemma we collect some elementary properties of the maps $\widetilde{\sigma}_{\left(w, w^{\prime}\right)}$ and $\widetilde{\sigma}_{\iota}$. First we introduce some auxiliary notations.

For a regular function $g \in \mathbb{C}[T]$, we write $g(x) \in \mathbb{C}[T \times T]$ (respectively $g(y) \in$ $\mathbb{C}[T \times T])$ for the corresponding regular function on $T \times T$ constant with respect to the second (respectively first) $T$-component. In particular, the $x_{i}$ (respectively $\left.y_{i}\right)$ are the standard coordinate functions of the first (respectively second) copy of $T$ in $T \times T$. Recall the regular function $d_{w} \in \mathbb{C}[T](w \in W)$ such that

$$
\widetilde{S}_{w}=d_{w}(X) w
$$

in $\mathbb{C}(T) \# W$; see (2.7).

Lemma 3.1. The complex linear endomorphisms $\widetilde{\sigma}_{\left(w, w^{\prime}\right)}$ and $\widetilde{\sigma}_{\iota}$ of $\mathbb{H}$ satisfy the following properties:

(i) the $\widetilde{\sigma}_{\left(s_{i}, e\right)}\left(i \in \mathbb{Z}_{N}\right)$ satisfy the $\widehat{A}_{N-1}$ braid relations;

(ii) $\widetilde{\sigma}_{\left(s_{i}, e\right)}^{2}=d_{s_{i}}(x)\left(s_{i} d_{s_{i}}\right)(x) \cdot$ id for $i \in \mathbb{Z}_{N}$;

(iii) $\widetilde{\sigma}_{(\pi, e)} \widetilde{\sigma}_{\left(s_{i}, e\right)} \widetilde{\sigma}_{\left(\pi^{-1}, e\right)}=\widetilde{\sigma}_{\left(s_{i+1}, e\right)}$ for $i \in \mathbb{Z}_{N}$;

(iv) $\widetilde{\sigma}_{\iota}^{2}=\mathrm{id}$ and $\widetilde{\sigma}_{(e, w)}=\widetilde{\sigma}_{\iota} \widetilde{\sigma}_{(w, e)} \widetilde{\sigma}_{\iota}$ for $w \in W$; and

(v) $\widetilde{\sigma}_{(w, e)} \widetilde{\sigma}_{\left(e, w^{\prime}\right)}=\widetilde{\sigma}_{\left(w, w^{\prime}\right)}=\widetilde{\sigma}_{\left(e, w^{\prime}\right)} \widetilde{\sigma}_{(w, e)}$ for $w, w^{\prime} \in W$.

Proof. These are direct consequences of Proposition 2.9 and Theorem 2.8 ,

To construct a $\mathbb{W}$-action from the maps $\widetilde{\sigma}_{\left(w, w^{\prime}\right)}$ and $\widetilde{\sigma}_{\iota}$, we need to renormalize the maps appropriately. To do so, we describe as a first step the behavior of the maps $\widetilde{\sigma}_{\left(w, w^{\prime}\right)}$ and $\widetilde{\sigma}_{\iota}$ with respect to the $\mathbb{L}$-module structure (2.6) on $\mathbb{H}$. This will allow us to extend the maps $\widetilde{\sigma}_{\left(w, w^{\prime}\right)}$ and $\widetilde{\sigma}_{\iota}$ to endomorphisms of $H_{0}^{\mathbb{K}} \simeq \mathbb{K} \otimes_{\mathbb{L}} \mathbb{H}$, which is a suitably flexible surrounding for the normalizations of the maps to take place in.

Consider the group involution $\diamond: W \rightarrow W$ defined by $w^{\diamond}=w$ for $w \in S_{N}$ and $\lambda^{\diamond}=-\lambda$ for $\lambda \in \mathbb{Z}^{N}$. Then $\mathbb{W}$ acts on $T \times T$ by

$$
\begin{aligned}
\left(w, w^{\prime}\right)(t, \gamma) & =\left(w t, w^{\prime \diamond} \gamma\right), \\
\iota(t, \gamma) & =\left(\gamma^{-1}, t^{-1}\right)
\end{aligned}
$$

for $w, w^{\prime} \in W$, where $t^{-1}:=\left(t_{1}^{-1}, \ldots, t_{N}^{-1}\right) \in T$ and the action of $W$ on $T$ is by $q$-dilations and permutations; see (2.3). By transposition, this defines an action of $\mathbb{W}$ on $\mathbb{K}=\mathcal{M}(T \times T)$ by field automorphisms,

$$
(\mathrm{w} f)(t, \gamma)=f\left(\mathrm{w}^{-1}(t, \gamma)\right), \quad \mathrm{w} \in \mathbb{W} .
$$

Note that $\mathbb{L}=\mathbb{C}[T \times T]$ is a $\mathbb{W}$-subalgebra of $\mathbb{K}$.

Lemma 3.2. For $h \in \mathbb{H}$ and $f \in \mathbb{L}$ we have

$$
\begin{aligned}
\widetilde{\sigma}_{\left(w, w^{\prime}\right)}(f \cdot h) & =\left(\left(w, w^{\prime}\right) f\right) \cdot \widetilde{\sigma}_{\left(w, w^{\prime}\right)}(h), \\
\widetilde{\sigma}_{\iota}(f \cdot h) & =(\iota f) \cdot \widetilde{\sigma}_{\iota}(h)
\end{aligned}
$$

for $w, w^{\prime} \in W$. 
Proof. From Proposition 2.9 we know that $\widetilde{S}_{w} p(X)=(w p)(X) \widetilde{S}_{w}$ in $\mathbb{H}$ for $p \in \mathbb{C}[T]$ and $w \in W$. For $p \in \mathbb{C}[T]$ let $p^{\diamond} \in \mathbb{C}[T]$ be defined by $p^{\diamond}(t)=p\left(t^{-1}\right)$, then we also have

$$
p(Y) \widetilde{S}_{w^{\prime}}^{*}=\left(\widetilde{S}_{w^{\prime}} p^{\diamond}(X)\right)^{*}=\left(\left(w^{\prime} p^{\diamond}\right)(X) \widetilde{S}_{w^{\prime}}\right)^{*}=\widetilde{S}_{w^{\prime}}^{*}\left(w^{\prime} p^{\diamond}\right)^{\diamond}(Y)
$$

in $\mathbb{H}$. Hence for $p, r \in \mathbb{C}[T]$,

$$
\widetilde{\sigma}_{\left(w, w^{\prime}\right)}(p(X) h r(Y))=(w p)(X) \widetilde{S}_{w} h \widetilde{S}_{w^{\prime}}^{*}\left(w^{\prime} r^{\diamond}\right)^{\diamond}(Y) .
$$

The first formula of (3.2) now follows since $\left(w^{\prime} r^{\diamond}\right)^{\diamond}=w^{\prime} \diamond r$. The second is immediate from the definition of the duality anti-involution.

As a direct consequence of Lemma 3.2 the maps $\widetilde{\sigma}_{\left(w, w^{\prime}\right)}\left(w, w^{\prime} \in W\right)$ and $\widetilde{\sigma}_{\iota}$ uniquely extend to complex linear endomorphisms of $H_{0}^{\mathbb{K}} \simeq \mathbb{K} \otimes_{\mathbb{L}} \mathbb{H}$ such that (3.2) is valid for all $f \in \mathbb{K}$ and $h \in H_{0}^{\mathbb{K}}$. We keep the same notations $\widetilde{\sigma}_{\left(w, w^{\prime}\right)}$ and $\widetilde{\sigma}_{\iota}$ for these maps. Note that the properties of $\widetilde{\sigma}_{\left(w, w^{\prime}\right)}$ and $\widetilde{\sigma}_{\iota}$ as described in Lemma 3.1 also hold true as identities between endomorphisms of $H_{0}^{\mathbb{K}}$.

Theorem 3.3. There is a unique group homomorphism

$$
\tau: \mathbb{W} \rightarrow \mathrm{GL}_{\mathbb{C}}\left(H_{0}^{\mathbb{K}}\right)
$$

satisfying

$$
\begin{aligned}
\tau\left(w, w^{\prime}\right)(f) & =d_{w}(x)^{-1} d_{w^{\prime}}^{\diamond}(y)^{-1} \cdot \widetilde{\sigma}_{\left(w, w^{\prime}\right)}(f), \\
\tau(\iota)(f) & =\widetilde{\sigma}_{\iota}(f)
\end{aligned}
$$

for $w, w^{\prime} \in W$ and $f \in H_{0}^{\mathbb{K}}$. It satisfies $\tau(\mathrm{w})(g \cdot f)=\mathrm{w} g \cdot \tau(\mathrm{w})(f)$ for $g \in \mathbb{K}$, $f \in H_{0}^{\mathbb{K}}$ and $\mathrm{w} \in \mathbb{W}$.

Proof. The last statement is clear.

The action $\tau$ of $W \times\{e\}$ arises naturally from left multiplication by normalized affine intertwiners on a suitable localization of the double affine Hecke algebra (see Cherednik [9, §1.3]). In the present set-up, one observes that Lemma 3.2 and Lemma 3.1(i)-(ii) imply that the $\tau\left(s_{i}, e\right)\left(i \in \mathbb{Z}_{N}\right)$ satisfy the $\widehat{A}_{N-1}$ braid relations and the quadratic relations $\tau\left(s_{i}, e\right)^{2}=\operatorname{id}_{H_{0}^{\mathbb{K}}}$. Since furthermore $\tau(\pi, e)$ is a complex linear automorphism of $H_{0}^{\mathbb{K}}$ with inverse $\tau\left(\pi^{-1}, e\right)$, and $\tau(\pi, e) \tau\left(s_{i}, e\right) \tau\left(\pi^{-1}, e\right)=$ $\tau\left(s_{i+1}, e\right)$ for $i \in \mathbb{Z}_{N}$ by Lemma 3.2 and Lemma 3.1(iii), we conclude that the formulas (3.3) for the maps $\tau\left(s_{i}, e\right)\left(i \in \mathbb{Z}_{N}\right)$ and $\tau(\pi, e)$ uniquely extend to a group homomorphism $\tau: W \times\{e\} \rightarrow \mathrm{GL}_{\mathbb{C}}\left(H_{0}^{\mathbb{K}}\right)$. It follows from Proposition 2.9 and its proof that the resulting group homomorphism satisfies

$$
\tau(w, e) f=d_{w}(x)^{-1} \cdot \widetilde{\sigma}_{(w, e)} f
$$

for $w \in W$. This is in accordance with formula (3.3).

Combining Lemma 3.1(iv) with Lemma 3.2 we can relate the complex endomorphism $\tau(e, w)$ (see (3.3) ) of $H_{0}^{\mathbb{K}}$ to $\tau(w, e)$ by the formula

$$
\tau(e, w)=\tau(\iota) \tau(w, e) \tau(\iota), \quad w \in W,
$$

where $\tau(\iota)$ is given by the second formula of (3.3). Since $\tau(\iota)^{2}=\widetilde{\sigma}_{\iota}^{2}=\mathrm{id}_{H_{0}^{\mathbb{K}}}$ we conclude that $W \ni w \mapsto \tau(e, w)$ (see (3.3) $)$ defines a left $W$-action on $H_{0}^{\mathbb{K}}$.

By Lemma 3.1 and Lemma 3.2 (v) we have

$$
\tau(w, e) \tau\left(e, w^{\prime}\right)=\tau\left(w, w^{\prime}\right)=\tau\left(e, w^{\prime}\right) \tau(w, e)
$$


for all $w, w^{\prime} \in W$. Thus $\tau: W \times W \rightarrow \mathrm{GL}_{\mathbb{C}}\left(H_{0}^{\mathbb{K}}\right)$, defined by the first formula of (3.3), is a group homomorphism. Combined with (3.4) and $\tau(\iota)^{2}=\operatorname{id}_{H_{0}^{\mathrm{K}}}$ we conclude that $\tau(3.3)$ indeed defines a complex linear action of $\mathbb{W}$ on $H_{0}^{\mathbb{K}}$.

For $\mathrm{w} \in \mathbb{W}$ and $f \in H_{0}^{\mathbb{K}}=\mathbb{K} \otimes H_{0}$ we write $\mathrm{w} f$ for the action of $\mathrm{w}$ on the $\mathbb{K}$-coefficients of $f$ in its expansion along a basis of $H_{0}$. In other words, viewing $f(t, \gamma)$ as $H_{0}$-valued meromorphic function in $(t, \gamma) \in T \times T$, the action is given by $(\mathrm{w} f)(t, \gamma)=f\left(\mathrm{w}^{-1}(t, \gamma)\right)$. Consider $\mathrm{GL}_{\mathbb{K}}\left(H_{0}^{\mathbb{K}}\right)$ as a $\mathbb{W}$-group by the corresponding conjugation action

$$
(\mathrm{w}, A) \mapsto \mathrm{w} A \mathrm{w}^{-1}, \quad \mathrm{w} \in \mathbb{W}, A \in \mathrm{GL}_{\mathbb{K}}\left(H_{0}^{\mathbb{K}}\right)
$$

by group automorphisms. We have the following direct consequence of the previous theorem.

Corollary 3.4. The map $\mathrm{w} \mapsto C_{\mathrm{w}}:=\tau(\mathrm{w}) \mathrm{w}^{-1}$ is a cocycle of $\mathbb{W}$ with values in the $\mathbb{W}$-group $\mathrm{GL}_{\mathbb{K}}\left(H_{0}^{\mathbb{K}}\right)$. In other words, $C_{\mathrm{w}} \in \mathrm{GL}_{\mathbb{K}}\left(H_{0}^{\mathbb{K}}\right)$ and

$$
C_{\mathrm{ww}^{\prime}}=C_{\mathrm{w}}{ }_{\mathrm{w}} C_{\mathrm{w}^{\prime} \mathrm{w}}{ }^{-1}
$$

for all $\mathrm{w}, \mathrm{w}^{\prime} \in \mathbb{W}$.

For more details on non-abelian group cohomology, see, e.g., the appendix in 43.

Remark 3.5. Interpreting $A \in \operatorname{End}_{\mathbb{K}}\left(H_{0}^{\mathbb{K}}\right)$ as $\operatorname{End}\left(H_{0}\right)$-valued meromorphic function $A(t, \gamma)$ in $(t, \gamma) \in T \times T$, the action (3.5) becomes $(\mathrm{w} A)(t, \gamma)=A\left(\mathrm{w}^{-1}(t, \gamma)\right)$. In particular, $\mathrm{w} A \mathrm{w}^{-1}=A$ for all $\mathrm{w} \in \mathbb{W}$ if $A \in \operatorname{End}_{\mathbb{K}}\left(H_{0}^{\mathbb{K}}\right)$ is the $\mathbb{K}$-linear extension of a complex linear endomorphism of $H_{0}$. This is, for instance, the case for the cocycle value $C_{\iota}$ (see Subsection 4.2).

Remark 3.6. One may replace in this subsection $\mathbb{K}$ by the field $\mathbb{C}(T \times T)$ of rational functions on $T \times T$. Consequently, the cocycle value $C_{\mathrm{w}}(t, \gamma)$ for $\mathrm{w} \in \mathbb{W}$ is a rational $\operatorname{End}\left(H_{0}\right)$-valued function in $(t, \gamma) \in T \times T$. We presented the results with respect to $\mathbb{K}=\mathcal{M}(T \times T)$ since this is the natural setting for the applications of the cocycle $C$ in the analytic theory of the quantum KZ equations (to which we come at a later stage).

3.2. Bispectral quantum $\mathbf{K Z}$ equations. In this subsection, we use the cocycle $C_{\mathrm{w}} \in \mathrm{GL}_{\mathbb{K}}\left(H_{0}^{\mathbb{K}}\right)(\mathrm{w} \in \mathbb{W})$ to define a holonomic system of $q$-difference equations on the space $H_{0}^{\mathbb{K}}$ of $H_{0}$-valued meromorphic functions on $T \times T$.

The constructions thus far have led to a $\mathbb{C}$-linear action $\tau$ of $\mathbb{W}$ on $H_{0}^{\mathbb{K}}$. In terms of the cocycle $C_{\mathrm{w}}(\mathrm{w} \in \mathbb{W})$, it is given by

$$
(\tau(\mathrm{w}) f)(t, \gamma)=C_{\mathrm{w}}(t, \gamma) f\left(\mathrm{w}^{-1}(t, \gamma)\right)
$$

for $\mathrm{w} \in \mathbb{W}$ and $f \in H_{0}^{\mathbb{K}}$, where (3.6) should be read as identities between $H_{0}$-valued meromorphic functions in $(t, \gamma) \in T \times T$. It follows that $f \in H_{0}^{\mathbb{K}}$ is $\tau\left(\mathbb{Z}^{N} \times \mathbb{Z}^{N}\right)$ invariant if and only if

$$
C_{(\lambda, \mu)}(t, \gamma) f\left(q^{-\lambda} t, q^{\mu} \gamma\right)=f(t, \gamma) \quad \forall \lambda, \mu \in \mathbb{Z}^{N},
$$

viewed as identities between $H_{0}$-valued meromorphic functions on $T \times T$.

Definition 3.7. We call the q-difference equations (3.7) the bispectral quantum $K Z$ (BqKZ) equations. We write SOL for the set of functions $f \in H_{0}^{\mathbb{K}}$ satisfying the BqKZ equations (3.7). 
Let $\mathbb{F} \subset \mathbb{K}$ denote the subfield consisting of $f \in \mathbb{K}$ satisfying $(\lambda, \mu) f=f$ for all $\lambda, \mu \in \mathbb{Z}^{N}$. Let furthermore $\mathbb{S}_{N}$ denote the subgroup $\mathbb{Z}_{2} \ltimes\left(S_{N} \times S_{N}\right)$ of $\mathbb{W}$.

Corollary 3.8. (i) The BqKZ equations (3.7) form a holonomic system of $q$ difference equations. In other words, the connection matrices $C_{(\lambda, \mu)}\left(\lambda, \mu \in \mathbb{Z}^{N}\right)$ satisfy the compatibility conditions

$$
C_{(\lambda, \mu)}(t, \gamma) C_{(\nu, \xi)}\left(q^{-\lambda} t, q^{\mu} \gamma\right)=C_{(\nu, \xi)}(t, \gamma) C_{(\lambda, \mu)}\left(q^{-\nu} t, q^{\xi} \gamma\right)
$$

for $\lambda, \mu, \nu, \xi \in \mathbb{Z}^{N}$, as $\operatorname{End}\left(H_{0}\right)$-valued meromorphic functions in $(t, \gamma) \in T \times T$.

(ii) The solution space $\mathrm{SOL}$ of $B q K Z$ is a $\tau\left(\mathbb{S}_{N}\right)$-invariant $\mathbb{F}$-subspace of $H_{0}^{\mathbb{K}}$.

Proof. (i) The cocycle condition implies that both sides of (3.8) are equal to $C_{(\lambda+\nu, \mu+\xi)}(t, \gamma)$

(ii) Clearly, SOL is a $\mathbb{F}$-subspace of $H_{0}^{\mathbb{K}}$. Note, furthermore, that $\mathbb{Z}^{N} \times \mathbb{Z}^{N}$ is a normal subgroup of $\mathbb{W}$ with quotient group isomorphic to $\mathbb{S}_{N}$. Hence the $\mathbb{F}$ subspace SOL of $\tau\left(\mathbb{Z}^{N} \times \mathbb{Z}^{N}\right)$-invariant elements in the $\tau(\mathbb{W})$-module $H_{0}^{\mathbb{K}}$ is $\tau\left(\mathbb{S}_{N}\right)$ invariant.

\section{The EXPlicit FORM OF THE BISPECTRAL QUANTUM KZ EQUATIONS}

In this section we derive explicit expressions for the cocycle values $C_{\mathrm{w}}(\mathrm{w} \in \mathbb{W})$ and, in particular, for the $q$-connection matrices $C_{(\lambda, \mu)}\left(\lambda, \mu \in \mathbb{Z}^{N}\right)$ of the BqKZ. It will become apparent that the $C_{(\lambda, e)}(\cdot, \zeta)\left(\lambda \in \mathbb{Z}^{N}\right)$ with $\zeta \in T$ fixed coincide with the $q$-connection matrices of Cherednik's quantum affine KZ equation associated to the principal series module of $H(k)$ with central character $\zeta$. They also turn up as gauged $q$-connection matrices for a Frenkel-Reshetikhin [20] type quantum KZ equation associated to the quantum affine algebra $\mathcal{U}_{k}\left(\widehat{s l}_{N}\right)$.

4.1. Formal principal series. View the commutative subalgebra $\mathbb{C}_{Y}[T]$ of $H$ as left $\mathbb{C}_{Y}[T]$-module by left multiplication. Let $M=\operatorname{Ind}_{\mathbb{C}_{Y}[T]}^{H}\left(\mathbb{C}_{Y}[T]\right)$ be the corresponding induced left $H$-module. With respect to the $\mathbb{C}[T] \simeq \mathbb{C}[\{1\} \times T]$ module structure

$$
f \cdot\left(h \otimes_{\mathbb{C}_{Y}[T]} g(Y)\right)=h \otimes_{\mathbb{C}_{Y}[T]}(f g)(Y) \quad f, g \in \mathbb{C}[T], h \in H
$$

on $M$ we have $M \simeq H_{0}^{\mathbb{C}[\{1\} \times T]}=\mathbb{C}[\{1\} \times T] \otimes H_{0}$ as $\mathbb{C}[\{1\} \times T]$-modules. The left $H$-action on $M$ is $\mathbb{C}[\{1\} \times T]$-linear, hence we obtain an algebra homomorphism

$$
\eta: H \rightarrow \operatorname{End}_{\mathbb{C}[\{1\} \times T]}\left(H_{0}^{\mathbb{C}[\{1\} \times T]}\right) .
$$

We occasionally view $\eta(h)$ as $\operatorname{End}\left(H_{0}\right)$-valued regular function in $\gamma \in T$, in which case we write it as $T \ni \gamma \mapsto \eta(h)(\gamma)$. Extending the ground ring $\mathbb{C}[\{1\} \times T]$ to $\mathbb{K}=\mathcal{M}(T \times T)$ we obtain an algebra homomorphism

$$
H \rightarrow \operatorname{End}_{\mathbb{K}}\left(H_{0}^{\mathbb{K}}\right),
$$

which we shall also denote by $\eta$. From this viewpoint, $\eta(h)(\gamma)$ is the regular $\operatorname{End}\left(H_{0}\right)$-valued function in $(t, \gamma) \in T \times T$ which is constant in $t$. Note that $\eta(h)$ for $h \in H_{0}$ is constant as $\operatorname{End}\left(H_{0}\right)$-valued function on $T \times T$ (see Remark 3.5).

Lemma 4.1. For $w \in S_{N}$ and $1 \leq i<N$ we have

$$
\eta\left(T_{i}\right) T_{w}= \begin{cases}T_{s_{i} w} & \text { if } \ell\left(s_{i} w\right)=\ell(w)+1, \\ \left(k-k^{-1}\right) T_{w}+T_{s_{i} w} & \text { if } \ell\left(s_{i} w\right)=\ell(w)-1,\end{cases}
$$


and

$$
\eta(\pi)(\gamma) T_{w}=\gamma_{w^{-1}(N)} T_{\sigma w}
$$

as regular $H_{0}$-valued functions in $\gamma \in T$.

Proof. The first formula follows directly from the definitions. For the second formula it suffices to verify that $\pi T_{w}=T_{\sigma w} Y_{w^{-1}(N)}$ in $H$.

If $w=1$ then $\pi=T_{\sigma} Y_{N}$ since $\sigma=s_{1} s_{2} \cdots s_{N-1}$ is a reduced expression. If $w=s_{N-1}$ then

$$
\pi T_{w}=\pi T_{N-1}=T_{1} \cdots T_{N-2} Y_{N-1}=T_{s_{1} \cdots s_{N-2}} Y_{w^{-1}(N)}=T_{\sigma w} Y_{w^{-1}(N)} .
$$

Next, we prove that $\pi T_{w}=T_{\sigma w} Y_{w^{-1}(N)}$ in $H$ if $w \neq 1$ and $\ell\left(s_{i} w\right)=\ell(w)+1$ for all $1 \leq i \leq N-2$. Then $w=s_{N-1} s_{N-2} \cdots s_{j}$ for some $1 \leq j<N$ (and this is a reduced expression of $w$ ). We find, making repetitive use of the cross relation $T_{r} Y_{r+1} T_{r}=Y_{r}(1 \leq r<N)$ in $H$,

$$
\begin{aligned}
\pi T_{w} & =\pi T_{N-1} T_{N-2} \cdots T_{j}=T_{1} \cdots T_{N-2} Y_{N-1} T_{N-2} \cdots T_{j} \\
& =T_{1} \cdots T_{N-3} Y_{N-2} T_{N-3} \cdots T_{j} \\
& \vdots \\
& =T_{1} \cdots T_{j-1} Y_{j}=T_{s_{1} \cdots s_{j-1}} Y_{j} \\
& =T_{\sigma w} Y_{j}=T_{\sigma w} Y_{w^{-1}(N)}
\end{aligned}
$$

which is the desired relation in $H$.

The general case is now proved by induction on $\ell(w)$. Let $w \neq 1$ and decompose it as $w=s_{i} u$ with $1 \leq i<N$ and $u \in S_{N}$ such that $\ell\left(s_{i} u\right)=\ell(u)+1$. Suppose that $\pi T_{u}=T_{\sigma u} Y_{u^{-1}(N)}$ in $H$. In order to prove that $\pi T_{w}=T_{\sigma w} Y_{w^{-1}(N)}$ we may, in view of the previous paragraph, assume without loss of generality that $1 \leq i \leq N-2$. Then $s_{i}(N)=N$ and $\ell\left(s_{i+1} \sigma u\right)=\ell(\sigma u)+1$. (The latter equality is equivalent to $(\sigma u)^{-1}(i+1)<(\sigma u)^{-1}(i+2)$, which follows from $u^{-1}(i)<u^{-1}(i+1)$, which again is equivalent to the assumption $\ell\left(s_{i} u\right)=\ell(u)+1$.) Then

$$
\begin{aligned}
\pi T_{w} & =\pi T_{i} T_{u}=T_{i+1} \pi T_{u}=T_{i+1} T_{\sigma u} Y_{u^{-1}(N)} \\
& =T_{s_{i+1} \sigma u} Y_{u^{-1}} s_{i}(N)=T_{\sigma s_{i} u} Y_{w^{-1}(N)} \\
& =T_{\sigma w} Y_{w^{-1}(N)}
\end{aligned}
$$

in $H$, which completes the proof.

In view of the explicit expression (2.8) for the intertwiner $\widetilde{S}_{i} \in \mathbb{H}(1 \leq i<N)$ and the definition of the duality anti-involution, we have $\widetilde{S}_{w}^{*} \in H$ for all $w \in S_{N}$. We now set

$$
\xi_{w}:=\eta\left(\widetilde{S}_{w^{-1}}^{*}\right) T_{e} \in H_{0}^{\mathbb{K}}, \quad w \in S_{N} .
$$

Note that $\xi_{w} \in H_{0}^{\mathbb{C}[\{1\} \times T]} \subset H_{0}^{\mathbb{K}}$ for $w \in S_{N}$. We view $\xi_{w}$ as regular $H_{0}$-valued function in $\gamma \in T$, as well as meromorphic $H_{0}$-valued function in $(t, \gamma) \in T \times T$ constant in $t \in T$.

Lemma 4.2. $\left\{\xi_{w}\right\}_{w \in S_{N}}$ is a $\mathbb{K}$-basis of $H_{0}^{\mathbb{K}}$ consisting of common eigenfunctions for the $\eta$-action of $\mathbb{C}_{Y}[T]$ on $H_{0}^{\mathbb{K}}$. For $p \in \mathbb{C}[T]$ and $w \in S_{N}$ we have

$$
\eta(p(Y))(\gamma) \xi_{w}(\gamma)=\left(w^{-1} p\right)(\gamma) \xi_{w}(\gamma)
$$

as $H_{0}$-valued regular functions in $\gamma \in T$. 
Proof. For $p \in \mathbb{C}[T]$ we have $\eta(p(Y))(\gamma) T_{e}=p(\gamma) T_{e}$. Note, furthermore, that $p(Y) \widetilde{S}_{w^{-1}}^{*}=\widetilde{S}_{w^{-1}}^{*}\left(w^{-1} p\right)(Y)$ in $H$ for $p \in \mathbb{C}[T]$ and $w \in S_{N}$; see the proof of Lemma 3.2. Combining the two observations gives (4.3). It follows from Proposition 2.9(iv)-(v) that the $\xi_{w}\left(w \in S_{N}\right)$ are nonzero in $H_{0}^{\mathbb{K}}$. The eigenvalue equations (4.3) then show that the $\xi_{w}\left(w \in S_{N}\right)$ are $\mathbb{K}$-linearly independent in $H_{0}^{\mathbb{K}}$.

4.2. The cocycle values. We define

$$
R_{i}(z)=c_{k}(z)^{-1}\left(\eta\left(T_{i}\right)-k\right)+1, \quad 1 \leq i<N
$$

viewed as a rational $\operatorname{End}\left(H_{0}\right)$-valued function in $z$. The results of the previous subsection implies that the $R_{i}(z)$ satisfy the following Yang-Baxter type equations (see Cherednik [9, \$1.3.2]).

Lemma 4.3. We have

$$
C_{\left(s_{i}, e\right)}(t, \gamma)=R_{i}\left(t_{i} / t_{i+1}\right), \quad 1 \leq i<N
$$

as rational $\operatorname{End}\left(H_{0}\right)$-valued functions in $(t, \gamma) \in T \times T$. In particular, the $R_{i}(z)$ satisfy

$$
\begin{aligned}
R_{i}(z) R_{i}\left(z^{-1}\right) & =\mathrm{id}, \\
R_{j}(z) R_{j+1}\left(z z^{\prime}\right) R_{j}\left(z^{\prime}\right) & =R_{j+1}\left(z^{\prime}\right) R_{j}\left(z z^{\prime}\right) R_{j+1}(z),
\end{aligned}
$$

for $1 \leq i<N$ and $1 \leq j<N-1$ as $\operatorname{End}\left(H_{0}\right)$-valued rational functions.

Proof. For $1 \leq i<N$ and $h \in H_{0}$ we have, as $H_{0}$-valued meromorphic functions in $(t, \gamma) \in T \times T$

$$
\begin{aligned}
C_{\left(s_{i}, e\right)}(t, \gamma) h & =\left(\tau\left(s_{i}, e\right) h\right)(t, \gamma) \\
& =d_{s_{i}}(t)^{-1}\left(\widetilde{S}_{i} h\right)(t, \gamma) \\
& =c_{k}\left(t_{i} / t_{i+1}\right)^{-1}\left(\eta\left(T_{i}\right)-k\right) h+h,
\end{aligned}
$$

in view of the explicit expression (2.8) for $\widetilde{S}_{i}, c_{k}$ (2.5) and $d_{s_{i}}$ (see the proof of Lemma 2.9). For the second statement of the lemma, note that the cocycle property of $C$ implies for $1 \leq i<N$ and $1 \leq j<N-1$ that

$$
\begin{aligned}
C_{\left(s_{i}, e\right)}(t, \gamma) C_{\left(s_{i}, e\right)}\left(s_{i} t, \gamma\right) & =\mathrm{id}, \\
C_{\left(s_{j}, e\right)}(t, \gamma) C_{\left(s_{j+1}, e\right)}\left(s_{j} t, \gamma\right) & C_{\left(s_{j}, e\right)}\left(s_{j+1} s_{j} t, \gamma\right) \\
& =C_{\left(s_{j+1}, e\right)}(t, \gamma) C_{\left(s_{j}, e\right)}\left(s_{j+1} t, \gamma\right) C_{\left(s_{j+1}, e\right)}\left(s_{j} s_{j+1} t, \gamma\right)
\end{aligned}
$$

as rational $\operatorname{End}\left(H_{0}\right)$-valued functions in $(t, \gamma) \in T \times T$. Using $C_{\left(s_{i}, e\right)}(t, \gamma)=$ $R_{i}\left(t_{i} / t_{i+1}\right)$ these formulas imply (4.4).

Observe that $C_{\iota}$ is the $\mathbb{K}$-linear extension of the anti-algebra involution of $H_{0}$ mapping $T_{w}$ to $T_{w^{-1}}$ for all $w \in S_{N}$. Note furthermore that

$$
C_{(\pi, e)}=\eta(\pi) .
$$

Together with the explicit description of $C_{\left(s_{i}, e\right)}(1 \leq i<N)$ from the previous lemma, these formulas determine the values $C_{\mathrm{w}}(\mathrm{w} \in \mathbb{W}$ ) uniquely (cf. Corollary 3.4). In particular, the cocycle property of $C$ implies that

$$
C_{(e, w)}(t, \gamma)=C_{\iota} C_{(w, e)}\left(\gamma^{-1}, t^{-1}\right) C_{\iota}, \quad w \in W,
$$

as $\operatorname{End}\left(H_{0}\right)$-valued rational functions in $(t, \gamma) \in T \times T$. 
Lemma 4.4. Let $w \in W$.

(i) $C_{(w, e)} \in(\mathbb{C}(T) \otimes \mathbb{C}[T]) \otimes \operatorname{End}\left(H_{0}\right)$.

(ii) The $\mathbb{C}[T] \otimes \operatorname{End}\left(H_{0}\right)$-valued rational function $t \mapsto C_{(w, e)}(t, \cdot)$ in $t \in T$ is regular at $t \in T \backslash \mathcal{S}$, where

$$
\mathcal{S}=\left\{t \in T \mid t^{\alpha} \in k^{-2} q^{\mathbb{Z}} \quad \text { for some } \alpha \in R\right\} .
$$

Proof. By the cocycle condition, $C_{(w, e)}(t, \gamma)$ can be written as a product of factors $C_{\left(s_{i}, e\right)}(u t, \gamma)(1 \leq i<N, u \in W)$ and $\eta\left(\pi^{ \pm 1}\right)(\gamma)$. By Lemma 4.1 and the fact that $R_{i}(z)$ has a single pole at $z=k^{-2}$, we conclude (i) and (ii).

4.3. The $q$-connection matrices. Besides the standard $\mathbb{Z}$-basis $\left\{\epsilon_{i}\right\}_{i=1}^{N}$ of $\mathbb{Z}^{N}$, we also have the $\mathbb{Z}$-basis $\left\{\varpi_{i}\right\}_{i=1}^{N}$ consisting of the fundamental weights $\varpi_{i}:=$ $\sum_{j=1}^{i} \epsilon_{j} \in \mathbb{Z}^{N}$. Note that

$$
\varpi_{i}=\pi^{i} \sigma^{-i}
$$

in $W$ for all $1 \leq i \leq N$. In the following lemma, we compute the $q$-connection matrices $C_{(\lambda, e)}$ for $\lambda \in \mathbb{Z}^{N}$ of $\mathrm{BqKZ}$ explicitly in case $\lambda$ is one of these two types of basis elements of $\mathbb{Z}^{N}$.

Lemma 4.5. (i) For $1 \leq j \leq N$ we have

$$
\begin{aligned}
C_{\left(\epsilon_{j}, e\right)}(t, \gamma) & =R_{j-1}\left(t_{j-1} / t_{j}\right) R_{j-2}\left(t_{j-2} / t_{j}\right) \cdots R_{1}\left(t_{1} / t_{j}\right) \\
& \times \eta(\pi)(\gamma) R_{N-1}\left(q t_{N} / t_{j}\right) \cdots R_{j+1}\left(q t_{j+2} / t_{j}\right) R_{j}\left(q t_{j+1} / t_{j}\right)
\end{aligned}
$$

as rational $\operatorname{End}\left(H_{0}\right)$-valued functions in $(t, \gamma) \in T \times T$.

(ii) For $1 \leq i<N$ we have

$$
\begin{aligned}
C_{\left(\varpi_{i}, e\right)}(t, \gamma)=( & \eta(\pi)(\gamma))^{i}\left(R_{N-i}\left(q t_{N} / t_{1}\right) \cdots R_{2}\left(q t_{i+2} / t_{1}\right) R_{1}\left(q t_{i+1} / t_{1}\right)\right) \\
\times & \cdots \times\left(R_{N-2}\left(q t_{N} / t_{i-1}\right) \cdots R_{i}\left(q t_{i+2} / t_{i-1}\right) R_{i-1}\left(q t_{i+1} / t_{i-1}\right)\right) \\
& \times\left(R_{N-1}\left(q t_{N} / t_{i}\right) \cdots R_{i+1}\left(q t_{i+2} / t_{i}\right) R_{i}\left(q t_{i+1} / t_{i}\right)\right)
\end{aligned}
$$

as rational $\operatorname{End}\left(H_{0}\right)$-valued functions in $(t, \gamma) \in T \times T$.

(iii) We have

$$
C_{\left(\varpi_{N}, e\right)}(t, \gamma)=\gamma^{\varpi_{N}} \mathrm{id}
$$

as rational $\operatorname{End}\left(H_{0}\right)$-valued functions in $(t, \gamma) \in T \times T$.

Proof. (i) By the cocycle property of $C$ and by the expression (2.2) for $\epsilon_{j} \in W$, we obtain an explicit expression for $C_{\left(\epsilon_{j}, e\right)}$ in terms of the $C_{\left(s_{i}, e\right)}(1 \leq i<N)$ and $C_{(\pi, e)}$. Combining (4.5) and the previous lemma then gives the desired expression for $C_{\left(\epsilon_{j}, e\right)}(t, \gamma)$.

(ii) $\sigma^{i}$ is the permutation

$$
\left(\begin{array}{cccccccc}
1 & 2 & \cdots & N-i & N-i+1 & N-i+2 & \cdots & N \\
i+1 & i+2 & \cdots & N & 1 & 2 & \cdots & i
\end{array}\right)
$$

so we find a reduced expression

$$
\sigma^{i}=\left(s_{i} \cdots s_{N-1}\right)\left(s_{i-1} \cdots s_{N-2}\right) \cdots\left(s_{2} \cdots s_{N-i+1}\right)\left(s_{1} \cdots s_{N-i}\right) .
$$

Combined with (4.7) we get a reduced expression for $\varpi_{i}$. Using the cocycle condition for $C_{\mathrm{w}}$ repeatedly, we get the desired result.

(iii) Since $\sigma^{N}=1$, we get $C_{\left(\varpi_{N}, e\right)}(t, \gamma)=(\eta(\pi)(\gamma))^{N}$, which maps $T_{w}$ to $\gamma^{\varpi_{N}} T_{w}$ for all $w \in S_{N}$ in view of Lemma 4.1. 
We end this subsection by computing the asymptotic leading terms of the $q$ connection matrices $C_{(\lambda, e)}(t, \gamma)\left(\lambda \in \mathbb{Z}^{N}\right)$ as $\left|t^{-\alpha_{i}}\right| \rightarrow 0(1 \leq i<N)$, where we take $\alpha_{i}:=\epsilon_{i}-\epsilon_{i+1}(1 \leq i<N)$ as a base of the root system $R=\left\{\epsilon_{i}-\epsilon_{j}\right\}_{1 \leq i \neq j \leq N}$ of type $A_{N-1}$. Let $R_{+}=\left\{\epsilon_{i}-\epsilon_{j}\right\}_{1 \leq i<j \leq N}$ denote the associated set of positive roots and $Q_{+}=\bigoplus_{i=1}^{N-1} \mathbb{Z}_{\geq 0} \alpha_{i}$ the corresponding cone in the root lattice $Q$ of $R$. Let furthermore $\delta:=(N-1, N-3, \ldots, 1-N) \in \mathbb{Z}^{N}$ and write $w_{0} \in S_{N}$ for the longest Weyl group element (mapping $i$ to $N-i+1$ for $1 \leq i \leq N$ ).

Consider the subring $\mathcal{A}:=\mathbb{C}\left[x^{-\alpha_{1}}, \ldots, x^{-\alpha_{N-1}}\right]$ of $\mathbb{C}[T \times\{1\}]=\mathbb{C}\left[x_{1}^{ \pm 1}, \ldots, x_{N}^{ \pm 1}\right] \subset$ $\mathbb{C}[T \times T]$. We write $Q(\mathcal{A})$ for its quotient field and $Q_{0}(\mathcal{A})$ for the subring of $Q(\mathcal{A})$ consisting of rational functions which are analytic at the point $x^{-\alpha_{i}}=0$ $(1 \leq i<N)$. We consider $Q_{0}(\mathcal{A}) \otimes \mathbb{C}[T]$ as a subring of $\mathbb{C}(T \times T)$ in the natural way. The first part of the following corollary is a refinement of Lemma 4.4(i) in case $w \in \mathbb{Z}^{N}$.

Corollary 4.6. Let $\lambda \in \mathbb{Z}^{N}$. We have

$$
\left.C_{(\lambda, e)} \in\left(Q_{0}(\mathcal{A}) \otimes \mathbb{C}[T]\right) \otimes \operatorname{End}\left(H_{0}\right)\right) .
$$

Writing

$$
C_{(\lambda, e)}^{(0)}=\left.C_{(\lambda, e)}\right|_{x^{-\alpha_{1}}=0, \ldots, x^{-\alpha_{N-1}=0}} \in \mathbb{C}[T] \otimes \operatorname{End}\left(H_{0}\right),
$$

we have $C_{(\lambda, e)}^{(0)}=k^{\langle\delta, \lambda\rangle} \eta\left(T_{w_{0}} Y^{w_{0}(\lambda)} T_{w_{0}}^{-1}\right)$, where $\langle\cdot, \cdot\rangle$ is the standard scalar product on $\mathbb{R}^{N}$.

Proof. To prove (4.9) it suffices, in view of the cocyle property of $C$, to verify (4.9) for $\lambda=\epsilon_{i}$. The statement then follows from Lemma 4.5(i), Lemma 4.1 and the explicit expression of $R_{i}(z)$.

Observe that $\lim _{z \rightarrow 0} R_{i}(z)=k \eta\left(T_{i}^{-1}\right)$ for $1 \leq i<N$. Combined with Lemma 4.5(i) and the explicit expression for $Y_{j}$ (see (2.4) ) we obtain for $1 \leq j \leq N$,

$$
C_{\left(\epsilon_{j}, e\right)}(t, \gamma) \rightarrow k^{2 j-N-1} \eta\left(Y_{j}\right)(\gamma)
$$

as $\left|t^{\alpha_{i}}\right| \rightarrow 0$ for all $1 \leq i<N$, hence

$$
C_{(\lambda, e)}(t, \gamma) \rightarrow k^{-\langle\delta, \lambda\rangle} \eta\left(Y^{\lambda}\right)(\gamma)
$$

as $\left|t^{\alpha_{i}}\right| \rightarrow 0$ for all $1 \leq i<N$. To derive the asymptotics of $C_{(\lambda, e)}(t, \gamma)$ as $\left|t^{-\alpha_{i}}\right| \rightarrow 0$ for $1 \leq i<N$ we use the cocycle property to write

$$
C_{(\lambda, e)}(t, \gamma)=C_{\left(w_{0}, e\right)}(t, \gamma) C_{\left(w_{0}(\lambda), e\right)}\left(w_{0} t, \gamma\right) C_{\left(w_{0}, e\right)}\left(q^{-w_{0}(\lambda)} w_{0} t, \gamma\right) .
$$

Note that $C_{\left(w_{0}, e\right)}(t, \gamma) \rightarrow k^{\ell\left(w_{0}\right)} \eta\left(T_{w_{0}}^{-1}\right)$ if $\left|t^{\alpha_{i}}\right| \rightarrow 0$ for all $1 \leq i<N$. Hence

$$
C_{(\lambda, e)}^{(0)}=k^{\langle\delta, \lambda\rangle} \eta\left(T_{w_{0}} Y^{w_{0}(\lambda)} T_{w_{0}}^{-1}\right),
$$

as desired.

4.4. Relation to quantum KZ equations. Fix $\zeta \in T$ and let $\chi_{\zeta}: \mathbb{C}[\{1\} \times T] \rightarrow \mathbb{C}$ denote the corresponding evaluation character $\chi_{\zeta}(f)=f(\zeta)$. Recall the formal principal series $\eta: H \rightarrow \operatorname{End}_{\mathbb{C}[\{1\} \times T]}(M)$. The corresponding complex $H$ representation $M(\zeta)=\mathbb{C} \otimes_{\chi_{\zeta}} M$ of dimension $N$ ! is the principal series module of $H$ with central character $\zeta$. We identify $M(\zeta)$ with $H_{0}$ as a complex vector space, and push the $H$-action on $M(\zeta)$ through the linear isomorphism to $H_{0}$. We denote the corresponding representation map by

$$
\eta_{\zeta}: H \rightarrow \operatorname{End}\left(H_{0}\right)
$$


As in Subsection 4.1 we have as identities in $H_{0}$,

$$
\eta_{\zeta}\left(T_{i}\right) T_{w}= \begin{cases}T_{s_{i} w} & \text { if } \ell\left(s_{i} w\right)=\ell(w)+1, \\ \left(k-k^{-1}\right) T_{w}+T_{s_{i} w} & \text { if } \ell\left(s_{i} w\right)=\ell(w)-1,\end{cases}
$$

for $1 \leq i<N$ and $w \in S_{N}$,

$$
\eta_{\zeta}(\pi) T_{w}=\zeta_{w^{-1}(N)} T_{\sigma w}
$$

for $w \in S_{N}$, as well as

$$
\eta_{\zeta}(f(Y)) \xi_{w}(\zeta)=\left(w^{-1} f\right)(\zeta) \xi_{w}(\zeta)
$$

for $w \in S_{N}$, where $\xi_{w}(\zeta) \in H_{0}$ is the regular $H_{0}$-valued function $\xi_{w}(\gamma)$ in $\gamma \in$ $T$ specialized at $\gamma=\zeta$. Extending the base field to $\mathcal{M}(T)$ we get an algebra homomorphism $H \rightarrow \operatorname{End}_{\mathcal{M}(T)}\left(H_{0}^{\mathcal{M}(T)}\right)$, which is also denoted by $\eta_{\zeta}$.

In this subsection we consider the BqKZ for specialized values of $\gamma$. In view of Lemma 4.4(i) we may specialize $C_{(w, e)}(t, \gamma)(w \in W)$ at $\gamma=\zeta$. We write

$$
C_{w}^{\zeta}(t):=C_{(w, e)}(t, \zeta), \quad w \in W
$$

for the resulting specialized cocycle values, viewed as $\operatorname{End}\left(H_{0}\right)$-valued rational functions in $t \in T$. For $\zeta \in T$ the map $W \ni w \mapsto C_{w}^{\zeta}$ defines a cocycle of $W$ with values in the $W$-group $\mathrm{GL}_{\mathbb{C}(T)}\left(H_{0}^{\mathbb{C}(T)}\right)$. In other words,

$$
C_{w w^{\prime}}^{\zeta}(t)=C_{w}^{\zeta}(t) C_{w^{\prime}}^{\zeta}\left(w^{-1} t\right), \quad w, w^{\prime} \in W,
$$

as rational $H_{0}$-valued functions in $t \in T$. Comparing the cocycle values $C_{\lambda}^{\zeta}\left(\lambda \in \mathbb{Z}^{N}\right)$ to the ones in $[9, \S 1.3]$ we obtain the following result.

Corollary 4.7. Fix $\zeta \in T$. The holonomic system of q-difference equations

$$
C_{\lambda}^{\zeta}(t) f\left(q^{-\lambda} t\right)=f(t), \quad \forall \lambda \in \mathbb{Z}^{N}
$$

for $f \in H_{0}^{\mathcal{M}(T)}$ is Cherednik's quantum affine $K Z$ equation associated to the principal $H$-module $M(\zeta)$ with central character $\zeta$.

Let $\mathrm{SOL}_{\zeta} \subset H_{0}^{\mathcal{M}(T)}$ denote the set of solutions of the quantum KZ equations (4.10). Write $\mathcal{E}(T) \subset \mathcal{M}(T)$ for the subfield of meromorphic functions $f$ satisfying $f\left(q^{\lambda} t\right)=f(t)$ for all $\lambda \in \mathbb{Z}^{N}$ as meromorphic functions in $t \in T$. The set $\mathrm{SOL}_{\zeta}$ of solutions is a $\mathcal{E}(T)$-subspace of $H_{0}^{\mathcal{M}(T)}$. Furthermore, $\mathrm{SOL}_{\zeta}$ is invariant for the $S_{N}$-action

$$
(\varsigma(w) f)(t):=C_{w}^{\zeta}(t) f\left(w^{-1} t\right), \quad w \in S_{N}
$$

on $H_{0}^{\mathcal{M}(T)}$ (note that $\varsigma$ does not depend on $\zeta$ since $C_{w}^{\zeta}(t)=C_{(w, e)}(t, \zeta)$ is independent of $\zeta$ for $\left.w \in S_{N}\right)$.

Remark 4.8. The quantum KZ equations (4.10) are gauge equivalent to Frenkel and Reshetikhin's 20, quantum KZ equations associated with the $N$-fold tensor product representation $\mathbb{C}^{N}\left(t_{1}\right) \otimes \cdots \otimes \mathbb{C}^{N}\left(t_{N}\right)$ of the quantum affine algebra $\mathcal{U}_{k}\left(\widehat{s l}_{N}\right)$, where $\mathbb{C}^{N}\left(t_{i}\right)$ is the evaluation representation of the vector representation $\mathbb{C}^{N}$ of $\mathcal{U}_{k}\left(s l_{N}\right)$ (see [9, §1.3.2] and [14] for the details). 
In view of Corollary 4.7 the BqKZ equations (3.7) are a holonomic extension of the quantum KZ equations (4.10) by $q$-difference equations in the central character $\zeta$ of $M(\zeta)$. These may be thought of as analogs of isomonodromy transformations; in fact, in view of Lemma 4.2 and Corollary 4.6 the $q$-difference equations in $\zeta$ (which are essentially the quantum KZ equations again!) are reminiscent of Schlesinger transformations. This should be compared with the quantum isomonodromic interpretation of (rational) KZ equations as quantizations of Schlesinger equations, see [38] and [22].

From a different perspective we may think of the cocycle values $C_{(e, w)}(w \in W)$ as shift operators, in the sense that they map solutions of quantum KZ equations to solutions of quantum KZ equations with respect to shifted central characters. To formulate the precise result, we view in the following proposition $\gamma \mapsto C_{(e, w)}(\cdot, \gamma)$ as $\mathbb{C}[T] \otimes \operatorname{End}\left(H_{0}\right)$-valued rational function in $\gamma \in T$.

Proposition 4.9. Let $w \in W$ and $\zeta \in T$ such that $\gamma \mapsto C_{(e, w)}(\cdot, \gamma)$ is regular at $\gamma=\zeta$. Then $f \mapsto C_{(e, w)}(\cdot, \zeta) f$ defines an $S_{N}$-equivariant linear map $\mathrm{SOL}_{w} \diamond-1 \zeta \rightarrow$ $\mathrm{SOL}_{\zeta}$

Proof. By the cocycle property we have for $f \in \mathrm{SOL}_{w \diamond-1 \zeta}$ and $\lambda \in \mathbb{Z}^{N}$,

$$
\begin{aligned}
C_{\lambda}^{\zeta}(t)\left(C_{(e, w)}\left(q^{-\lambda} t, \zeta\right) f\left(q^{-\lambda} t\right)\right) & =C_{(\lambda, w)}(t, \zeta) f\left(q^{-\lambda} t\right) \\
& =C_{(e, w)}(t, \zeta) C_{\lambda}^{w^{\diamond-1} \zeta}(t) f\left(q^{-\lambda} t\right) \\
& =C_{(e, w)}(t, \zeta) f(t) .
\end{aligned}
$$

Hence $C_{(e, w)}(\cdot, \zeta) f \in \mathrm{SOL}_{\zeta}$. The $S_{N}$-equivariance of the map is again a consequence of the cocycle property of $C_{\mathrm{w}}(\mathrm{w} \in \mathbb{W})$; indeed, for $v \in S_{N}$ and $f \in \mathrm{SOL}_{w} \diamond-1 \zeta$ we have

$$
\begin{aligned}
C_{v}^{\zeta}(t)\left(C_{(e, w)}\left(v^{-1} t, \zeta\right) f\left(v^{-1} t\right)\right) & =C_{(v, w)}(t, \zeta) f\left(v^{-1} t\right) \\
& =C_{(e, w)}(t, \zeta)\left(C_{v}^{w^{\diamond-1} \zeta}(t) f\left(v^{-1} t\right)\right)
\end{aligned}
$$

which is the desired result.

From the quantum group perspective (see Remark 4.8), Proposition4.9 resembles the action of the dynamical Weyl group on solutions of quantum KZ equations from [18. We expect that the second half of the BqKZ is closely related to the VarchenkoEtingof dynamical difference equations [18, §9]; see also [19, [47, [44, 46], 45] and 29] for detailed studies of various degenerate cases. An interesting aspect, e.g., in [47] and [46], is the observation that $\mathrm{KZ}$ equations are dual to the associated dynamical equations using $\left(\mathfrak{g l}_{r}, \mathfrak{g l}_{s}\right)$ duality (our set-up relates to $r=s=N$ ). In the present theory this duality is incorporated by the cocycle value $C_{\iota}$, which relates the $q$-connection matrices $C_{(\lambda, e)}\left(\lambda \in \mathbb{Z}^{N}\right)$ of the quantum KZ equation to the dual $q$-connection matrices $C_{(e, \lambda)}$ by conjugation,

$$
C_{(e, \lambda)}(t, \gamma)=C_{\iota} C_{(\lambda, e)}\left(\gamma^{-1}, t^{-1}\right) C_{\iota}
$$

as $\operatorname{End}\left(H_{0}\right)$-valued meromorphic functions in $(t, \gamma) \in T \times T$. In turn, $C_{\iota}$ is a direct reflection of (the existence of) Cherednik's duality anti-isomorphism of the double affine Hecke algebra (see Theorem 2.8). 


\section{Solutions of the Bispectral QUANTUM KZ EQUATions}

In this section we use asymptotic analysis to construct a $\iota$-invariant solution $\Phi_{\kappa}$ of BqKZ, which we call the basic asymptotically free solution. It depends in a mild way on an auxiliary parameter $\kappa \in \mathbb{C}^{\times}$(in fact, $\mathbb{F} \Phi_{\kappa}$ is independent of $\kappa$ ). The orbit of $\Phi_{\kappa}$ under the action of $\{e\} \times S_{N} \subset \mathbb{S}_{N}$ turns out to be an $\mathbb{F}$-basis of SOL consisting of asymptotically free solutions. Along the way we derive various additional properties of $\Phi_{\kappa}$.

5.1. The leading term. Let $\theta \in \mathcal{M}(T)$ denote the renormalized Jacobi theta function

$$
\theta(z):=\prod_{m \geq 0}\left(1-q^{m} z\right)\left(1-q^{m+1} / z\right)
$$

for $z \in \mathbb{C}^{\times}$. It satisfies

$$
\theta\left(q^{m} z\right)=(-z)^{-m} q^{-\frac{1}{2} m(m-1)} \theta(z), \quad m \in \mathbb{Z} .
$$

For $\kappa \in \mathbb{C}^{\times}$we define $W_{\kappa} \in \mathbb{K}$ by

$$
W_{\kappa}(t, \gamma):=\prod_{i=1}^{N} \frac{\theta\left(\kappa t_{i} \gamma_{N-i+1}^{-1}\right)}{\theta\left(\kappa k^{\left\langle\delta, \epsilon_{i}\right\rangle} t_{i}\right) \theta\left(\kappa k^{-\left\langle\delta, \epsilon_{i}\right\rangle} \gamma_{N-i+1}^{-1}\right)} .
$$

By Corollary 4.6, the formal asymptotic form of the quantum $\mathrm{KZ}$ equations

$$
C_{(\lambda, e)}(t, \gamma) f\left(q^{-\lambda} t, \gamma\right)=f(t, \gamma), \quad \lambda \in \mathbb{Z}^{N}
$$

in the asymptotic region $\left|t^{\alpha_{i}}\right| \gg 0(1 \leq i<N)$ is

$$
k^{\langle\delta, \lambda\rangle} \eta\left(T_{w_{0}} Y^{w_{0}(\lambda)} T_{w_{0}}^{-1}\right)(\gamma) f\left(q^{-\lambda} t, \gamma\right)=f(t, \gamma), \quad \lambda \in \mathbb{Z}^{N}
$$

Lemma 5.1. $W_{\kappa} \in \mathbb{K}$ enjoys the following properties.

(i) $f_{\kappa}^{(0)}(t, \gamma):=W_{\kappa}(t, \gamma) T_{w_{0}}$ is a solution of (5.4).

(ii) $\iota\left(W_{\kappa}\right)=W_{\kappa}$ and $\tau(\iota) f_{\kappa}^{(0)}=f_{\kappa}^{(0)}$.

Proof. (i) Since $\eta\left(T_{w_{0}} Y^{w_{0}(\lambda)} T_{w_{0}}^{-1}\right)(\gamma) T_{w_{0}}=\gamma^{w_{0}(\lambda)} T_{w_{0}}$ for all $\lambda \in \mathbb{Z}^{N}$, it suffices to show that

$$
W_{\kappa}\left(q^{-\lambda} t, \gamma\right)=k^{-\langle\delta, \lambda\rangle} \gamma^{-w_{0}(\lambda)} W_{\kappa}(t, \gamma), \quad \lambda \in \mathbb{Z}^{N},
$$

which follows from (5.2).

(ii) Clearly $\iota\left(W_{\kappa}\right)=W_{\kappa}$, i.e. $W_{\kappa}\left(\gamma^{-1}, t^{-1}\right)=W_{\kappa}(t, \gamma)$. Since $C_{\iota}\left(T_{w_{0}}\right)=T_{w_{0}}$, it follows that $\tau(\iota) f_{\kappa}^{(0)}=f_{\kappa}^{(0)}$.

Observe that, more generally, $W_{\kappa} \in \mathbb{K}$ satisfies the $q$-difference equations

$$
W_{\kappa}\left(q^{-\lambda} t, q^{\mu} \gamma\right)=k^{-\langle\delta, \lambda+\mu\rangle} t^{w_{0}(\mu)} \gamma^{-w_{0}(\lambda)} q^{-\left\langle w_{0}(\lambda), \mu\right\rangle} W_{\kappa}(t, \gamma), \quad \lambda, \mu \in \mathbb{Z}^{N} .
$$

5.2. The basic asymptotically free solution $\Phi_{\kappa}$. We now gauge BqKZ by $W_{\kappa} \in$ $\mathbb{K}$. Concretely, for $\lambda, \mu \in \mathbb{Z}^{N}$ we write

$$
D_{(\lambda, \mu)}(t, \gamma)=W_{\kappa}(t, \gamma)^{-1} C_{(\lambda, \mu)}(t, \gamma) W_{\kappa}\left(q^{-\lambda} t, q^{\mu} \gamma\right)
$$

as $\operatorname{End}\left(H_{0}\right)$-valued meromorphic functions in $(t, \gamma) \in T \times T$. It is independent of $\kappa$ in view of (5.5). For $f \in H_{0}^{\mathbb{K}}$ we have $f \in$ SOL if and only if $g:=W_{\kappa}^{-1} f \in H_{0}^{\mathbb{K}}$ satisfies the holonomic system of $q$-difference equations

$$
D_{(\lambda, \mu)}(t, \gamma) g\left(q^{-\lambda} t, q^{\mu} \gamma\right)=g(t, \gamma), \quad \lambda, \mu \in \mathbb{Z}^{N}
$$

as $H_{0}$-valued rational functions in $(t, \gamma) \in T \times T$. 
The existence of a solution $\Psi \in H_{0}^{\mathbb{K}}$ of (5.6) admitting a convergent $H_{0}$-valued power series expansion

$$
\Psi(t, \gamma)=\sum_{\alpha, \beta \in Q_{+}} K_{\alpha, \beta} t^{-\alpha} \gamma^{\beta}, \quad K_{0,0}=T_{w_{0}}
$$

in the asymptotic region $\left|t^{\alpha_{i}}\right| \gg 0$ and $\left|\gamma^{-\alpha_{i}}\right| \gg 0(1 \leq i<N)$ is guaranteed by the following properties of the gauged $q$-connection matrices $D_{(\lambda, \mu)}$.

Consider the subring $\mathcal{B}:=\mathbb{C}\left[y^{\alpha_{1}}, \ldots, y^{\alpha_{N-1}}\right]$ of $\mathbb{C}[\{1\} \times T]=\mathbb{C}\left[y_{1}^{ \pm 1}, \ldots, y_{N}^{ \pm 1}\right]$. Write $Q(\mathcal{B})$ for its quotient field and $Q_{0}(\mathcal{B})$ for the subring of $Q(\mathcal{B})$ consisting of rational functions which are analytic at the point $y^{\alpha_{j}}=0(1 \leq j<N)$. We consider $Q_{0}(\mathcal{A}) \otimes \mathcal{B}$ and $\mathcal{A} \otimes Q_{0}(\mathcal{B})$ as subrings of $\mathbb{C}(T \times T)$ in the natural way.

Lemma 5.2. Set $A_{i}=D_{\left(\varpi_{i}, e\right)}$ and $B_{i}=D_{\left(e, \varpi_{i}\right)}$ for $1 \leq i \leq N$.

(i) $A_{N}=B_{N}=$ id on $H_{0}^{\mathbb{K}}$.

(ii) $A_{i} \in\left(Q_{0}(\mathcal{A}) \otimes \mathcal{B}\right) \otimes \operatorname{End}\left(H_{0}\right)$ and $B_{j} \in\left(\mathcal{A} \otimes Q_{0}(\mathcal{B})\right) \otimes \operatorname{End}\left(H_{0}\right)$.

(iii) Set $A_{i}^{(0,0)} \in \operatorname{End}\left(H_{0}\right)$ and $B_{j}^{(0,0)} \in \operatorname{End}\left(H_{0}\right)$ for the value of $A_{i}$ and $B_{j}$ at $x^{-\alpha_{r}}=0=y^{\alpha_{s}}(1 \leq r, s<N)$. For $w \in S_{N}$ we have

$$
A_{i}^{(0,0)}\left(T_{w_{0}} T_{w}\right)= \begin{cases}0 & \text { if } w^{-1} w_{0}\left(\varpi_{i}\right) \neq w_{0}\left(\varpi_{i}\right), \\ T_{w_{0}} T_{w} & \text { if } w^{-1} w_{0}\left(\varpi_{i}\right)=w_{0}\left(\varpi_{i}\right)\end{cases}
$$

and

$$
B_{i}^{(0,0)}\left(T_{w_{0}} T_{w}\right)= \begin{cases}0 & \text { if } w\left(\varpi_{i}\right) \neq \varpi_{i}, \\ T_{w_{0}} T_{w} & \text { if } w\left(\varpi_{i}\right)=\varpi_{i} .\end{cases}
$$

Proof. (i) We only give the proof of $A_{N}=$ id. Since $\varpi_{N}=\pi^{N}$ in $W$, we have

$$
A_{N}(t, \gamma)=W_{\kappa}(t, \gamma)^{-1} C_{\left(\varpi_{N}, e\right)}(t, \gamma) W_{\kappa}\left(q^{-\varpi_{N}} t, \gamma\right)=\gamma^{-\varpi_{N}}(\eta(\pi)(\gamma))^{N}=\mathrm{id}
$$

where we use (5.5) and (4.5) for the second equality, and Lemma 4.1 and $\sigma^{N}=e$ for the third equality.

(ii) Note that

$$
A_{i}(t, \gamma)=k^{-\left\langle\delta, \varpi_{i}\right\rangle} \gamma^{-w_{0}\left(\varpi_{i}\right)} C_{\left(\varpi_{i}, e\right)}(t, \gamma)
$$

by (5.5). Since $\varpi_{i}=\pi^{i} \sigma^{-i}$ the cocycle property of $C$ gives

$$
A_{i}(t, \gamma)=k^{-\left\langle\delta, \varpi_{i}\right\rangle} \gamma^{-w_{0}\left(\varpi_{i}\right)}(\eta(\pi)(\gamma))^{i} C_{\left(\sigma^{-i}, e\right)}\left(\pi^{-i} t, \gamma\right)
$$

It follows from the explicit expressions for the cocycle values $C_{\left(s_{i}, e\right)}(1 \leq i<N)$ that the $\operatorname{End}\left(H_{0}\right)$-valued rational function $C_{\left(\sigma^{-i}, e\right)}\left(\pi^{-i} t, \gamma\right)$ in $(t, \gamma) \in T \times T$ lies in $Q_{0}(\mathcal{A}) \otimes \operatorname{End}\left(H_{0}\right)$ (in particular, it is independent of $\gamma$ ). Furthermore, for $w \in S_{N}$

$$
\gamma^{-w_{0}\left(\varpi_{i}\right)}(\eta(\pi)(\gamma))^{i}\left(T_{w}\right)=\gamma^{w^{-1} w_{0}\left(\varpi_{i}\right)-w_{0}\left(\varpi_{i}\right)} T_{\sigma^{i} w}
$$

by Lemma 4.1. hence the $\operatorname{End}\left(H_{0}\right)$-valued regular function $\gamma^{-w_{0}\left(\varpi_{i}\right)}(\eta(\pi)(\gamma))^{i}$ in $\gamma \in T$ lies in $\mathcal{B} \otimes \operatorname{End}\left(H_{0}\right)$. Consequently, $A_{i} \in\left(Q_{0}(\mathcal{A}) \otimes \mathcal{B}\right) \otimes \operatorname{End}\left(H_{0}\right)$. The statement for $B_{j}$ follows from this using the cocycle property $C_{\left(e, \varpi_{j}\right)}(t, \gamma)=$ $C_{\iota} C_{\left(\varpi_{j}, e\right)}\left(\gamma^{-1}, t^{-1}\right) C_{\iota}$.

(iii) Recall that $\xi_{w}=\eta\left(\widetilde{S}_{w^{-1}}^{*}\right) T_{e}$ with $\widetilde{S}_{w}$ the intertwiners of $\mathbb{H}$ (see Proposition 2.9). By induction on $\ell(w)$, using the explicit expression (2.8) of the intertwiners 
$\widetilde{S}_{i}$, it follows that $\xi_{w} \in \mathcal{B} \otimes H_{0}$ and that the value of $\xi_{w}$ at $y^{\alpha_{i}}=0(1 \leq i<N)$ is $T_{w} \in H_{0}$. Set

$$
A_{i}^{(0)}=\left.A_{i}\right|_{x^{-\alpha_{1}}=0, \ldots, x^{-\alpha_{N-1}=0}} \in \mathcal{B} \otimes \operatorname{End}\left(H_{0}\right) .
$$

By Corollary 4.6 and (5.5),

$$
A_{i}^{(0)}=y^{-w_{0}\left(\varpi_{i}\right)} \eta\left(T_{w_{0}} Y^{w_{0}\left(\varpi_{i}\right)} T_{w_{0}}^{-1}\right) .
$$

Lemma 4.2 then gives

$$
A_{i}^{(0)}\left(\eta\left(T_{w_{0}}\right) \xi_{w}\right)=y^{w^{-1} w_{0}\left(\varpi_{i}\right)-w_{0}\left(\varpi_{i}\right)} \eta\left(T_{w_{0}}\right) \xi_{w}, \quad \forall w \in S_{N}
$$

as identities in $\mathcal{B} \otimes H_{0}$. Specializing (5.10) at $y^{\alpha_{j}}=0(1 \leq j<N)$ yields (5.8).

To prove (5.9) we consider

$$
\widetilde{B}_{j}^{(0)}=\left.B_{j}\right|_{y^{\alpha_{1}}=0, \ldots, y^{\alpha_{N-1}=0}} \in \mathcal{A} \otimes \operatorname{End}\left(H_{0}\right) .
$$

It is the rational $\operatorname{End}\left(H_{0}\right)$-valued function

$$
\widetilde{B}_{j}^{(0)}(t)=t^{w_{0}\left(\varpi_{j}\right)} C_{\iota}\left(\eta\left(T_{w_{0}} Y^{w_{0}\left(\varpi_{j}\right)} T_{w_{0}}^{-1}\right)\left(t^{-1}\right)\right) C_{\iota}
$$

in $t \in T$. Denoting $\widetilde{\xi}_{w} \in \mathcal{A} \otimes H_{0}$ for the rational $H_{0}$-valued function $\xi_{w}\left(t^{-1}\right)$ in $t \in T$, it follows that

$$
\widetilde{B}_{j}^{(0)}\left(C_{\iota} \eta\left(T_{w_{0}}\right) \widetilde{\xi}_{w}\right)=x^{-w^{-1} w_{0}\left(\varpi_{j}\right)+w_{0}\left(\varpi_{j}\right)} C_{\iota} \eta\left(T_{w_{0}}\right) \widetilde{\xi}_{w}
$$

for all $w \in S_{N}$. The value of $C_{\iota} \eta\left(T_{w_{0}}\right) \widetilde{\xi}_{w}$ at $x^{-\alpha_{i}}=0(1 \leq i<N)$ is $C_{\iota}\left(T_{w_{0}} T_{w}\right)$. In addition, $C_{\iota}$ restricts to the anti-algebra involution on $H_{0}$ mapping $T_{w}$ to $T_{w^{-1}}$ for $w \in S_{N}$, hence

$$
C_{\iota}\left(T_{w_{0}} T_{w}\right)=T_{w^{-1}} T_{w_{0}}=T_{w_{0}} T_{w_{0} w^{-1} w_{0}}
$$

Formula (5.9) then follows from specializing (5.11) at $x^{-\alpha_{i}}=0(1 \leq i<N)$ and replacing $w$ by $w_{0} w^{-1} w_{0}$ in the resulting formula.

For $\epsilon>0$, put $B_{\epsilon}:=\left\{t \in T|| t^{\alpha_{i}} \mid<\epsilon, \forall i\right\}$ and $B_{\epsilon}^{-1}:=\left\{t \in T \mid t^{-1} \in B_{\epsilon}\right\}$.

Theorem 5.3. There exists a unique solution $\Psi \in H_{0}^{\mathbb{K}}$ of the gauged equations (5.6) satisfying, for some $\epsilon>0$,

(i) $\Psi(t, \gamma)$ admits an $H_{0}$-valued power series expansion

$$
\Psi(t, \gamma)=\sum_{\alpha, \beta \in Q_{+}} K_{\alpha, \beta} t^{-\alpha} \gamma^{\beta}, \quad\left(K_{\alpha, \beta} \in H_{0}\right)
$$

for $(t, \gamma) \in B_{\epsilon}^{-1} \times B_{\epsilon}$ which is normally convergent on compacta of $B_{\epsilon}^{-1} \times B_{\epsilon}$. In particular, $\Psi(t, \gamma)$ is analytic at $(t, \gamma) \in B_{\epsilon}^{-1} \times B_{\epsilon}$;

(ii) $K_{0,0}=T_{w_{0}}$.

Proof. It follows from the previous lemma that the commuting endomorphisms $A_{i}^{(0,0)}, B_{j}^{(0,0)} \in \operatorname{End}\left(H_{0}\right)(1 \leq i, j<N)$ are semisimple. For $a, b \in \mathbb{C}^{N-1}$ set

$$
H_{0}[(a, b)]=\left\{v \in H_{0} \mid A_{i}^{(0,0)} v=a_{i} v \text { and } B_{j}^{(0,0)} v=b_{j} v(1 \leq i, j<N)\right\} \text {, }
$$

so that $H_{0}=\bigoplus_{(a, b) \in S} H_{0}[(a, b)]$ with $S$ the finite set of $(a, b) \in \mathbb{C}^{N-1} \times \mathbb{C}^{N-1}$ for which $H_{0}[(a, b)] \neq 0$. By the previous lemma, $\left(1^{N-1}, 1^{N-1}\right) \in S$ and we have $H_{0}\left[\left(1^{N-1}, 1^{N-1}\right)\right]=\operatorname{span}_{\mathbb{C}}\left\{T_{w_{0}}\right\}$. Furthermore, $a_{i}, b_{i} \notin q^{-\mathbb{N}}$ for all $(a, b) \in S$ and $i$. Under these conditions, the holonomic system of $q$-difference equations (5.6) admits a unique solution $\Psi$ satisfying the desired properties; see Theorem 8.6 in the appendix (to show that the gauged BqKZ falls in the class of holonomic systems of 
$q$-difference equations to which Theorem 8.6 applies, one should take $M=2(N-1)$, $q_{i}=q$ for $1 \leq i<N$ and variables $z_{i}=x^{-\alpha_{i}}$ and $z_{N-1+j}=y^{\alpha_{j}}$ for $1 \leq i, j<N$ in the appendix).

Remark 5.4. In a small neighborhood of a fixed $\left(t^{\prime}, \gamma^{\prime}\right) \in T \times T$, the meromorphic solution $\Psi$ of (5.6) can be expressed in terms of the power series expansion (5.12) by the formula

$$
\begin{aligned}
\Psi(t, \gamma) & =D_{(\lambda, \mu)}(t, \gamma) \Psi\left(q^{-\lambda} t, q^{\mu} \gamma\right) \\
& =D_{(\lambda, \mu)}(t, \gamma) \sum_{\alpha, \beta \in Q_{+}} K_{\alpha, \beta}\left(q^{-\lambda} t\right)^{-\alpha}\left(q^{\mu} \gamma\right)^{\beta}
\end{aligned}
$$

where $\lambda, \mu \in \mathbb{Z}^{N}$ are such that $\left(q^{-\lambda} t^{\prime}, q^{\mu} \gamma^{\prime}\right) \in B_{\epsilon}^{-1} \times B_{\epsilon}$.

Definition 5.5. We call $\Phi_{\kappa}:=W_{\kappa} \Psi \in$ SOL the basic asymptotically free solution of $B q K Z$.

Note that $\Phi_{\kappa} \in \mathbb{F}^{\times} \Phi_{\kappa^{\prime}}$ for $\kappa, \kappa^{\prime} \in \mathbb{C}^{\times}$. The $\kappa$-flexibility will come in handy when we consider specializations of $\Phi_{\kappa}$. In the following subsections, we derive various properties of the basic asymptotically free solution $\Phi_{\kappa}$.

\subsection{Duality.}

Theorem 5.6. The basic asymptotically free solution $\Phi_{\kappa}$ of $B q K Z$ is self-dual, in the sense that

$$
\tau(\iota) \Phi_{\kappa}=\Phi_{\kappa}
$$

Proof. SOL is $\mathbb{S}_{N}$-invariant, hence $\tau(\iota) \Phi_{\kappa} \in$ SOL. In addition,

$$
\tau(\iota) \Phi_{\kappa}=W_{\kappa}(\tau(\iota) \Psi)
$$

because $\iota\left(W_{\kappa}\right)=W_{\kappa}$. Hence $\tau(\iota) \Psi$ is a solution of the gauged equations (5.6) having a convergent $H_{0}$-valued power series expansion

$$
(\tau(\iota) \Psi)(t, \gamma)=C_{\iota} \Psi\left(\gamma^{-1}, t^{-1}\right)=\sum_{\alpha, \beta \in Q_{+}} C_{\iota}\left(K_{\alpha, \beta}\right) \gamma^{\alpha} t^{-\beta}
$$

for $(t, \gamma) \in B_{\epsilon}^{-1} \times B_{\epsilon}$. Since $C_{\iota}\left(K_{0,0}\right)=C_{\iota}\left(T_{w_{0}}\right)=T_{w_{0}}$, we conclude from Theorem 5.3 that $\tau(\iota) \Psi=\Psi$, hence $\tau(\iota) \Phi_{\kappa}=\Phi_{\kappa}$.

5.4. Singularities. Define

$$
\Lambda=\left\{\lambda \in \mathbb{Z}^{N} \mid \lambda_{1} \geq \lambda_{2} \geq \cdots \geq \lambda_{N}\right\}=\bigoplus_{i=1}^{N-1} \mathbb{Z}_{\geq 0} \varpi_{i} \oplus \mathbb{Z} \varpi_{N}
$$

i.e., $\Lambda$ consists of the $\lambda \in \mathbb{Z}^{N}$ such that $\langle\lambda, \alpha\rangle \in \mathbb{Z}_{\geq 0}$ for all $\alpha \in R_{+}$. Set

$$
\mathcal{S}_{+}:=\left\{t \in T \mid t^{\alpha} \in k^{-2} q^{-\mathbb{N}} \text { for some } \alpha \in R_{+}\right\} .
$$

Write $\Psi(t, \gamma)=\sum_{\alpha \in Q_{+}} \Gamma_{\alpha}(\gamma) t^{-\alpha}$ for $(t, \gamma) \in B_{\epsilon}^{-1} \times B_{\epsilon}$, where $\Gamma_{\alpha}$ is the $H_{0}$-valued analytic function on $B_{\epsilon}$ defined by the $H_{0}$-valued power series

$$
\Gamma_{\alpha}(\gamma):=\sum_{\beta \in Q_{+}} K_{\alpha, \beta} \gamma^{\beta}
$$


Lemma 5.7. The $\Gamma_{\alpha}\left(\alpha \in Q_{+}\right)$extend uniquely to a meromorphic $H_{0}$-valued function on $T$, analytic at $T \backslash \mathcal{S}_{+}$, such that $\Psi(t, \gamma)$ admits an $H_{0}$-valued power series expansion

$$
\Psi(t, \gamma)=\sum_{\alpha \in Q_{+}} \Gamma_{\alpha}(\gamma) t^{-\alpha}
$$

for $(t, \gamma) \in B_{\epsilon}^{-1} \times T \backslash \mathcal{S}_{+}$, converging normally on compacta of $B_{\epsilon}^{-1} \times T \backslash \mathcal{S}_{+}$.

Proof. Using Lemma 5.2 we write for $\mu \in \Lambda$,

$$
D_{(e, \mu)}(t, \gamma)=\sum_{\beta \in Q_{+}} F_{\beta}^{\mu}(\gamma) t^{-\beta}
$$

with $F_{\beta}^{\mu} \in Q_{0}(\mathcal{B}) \otimes \operatorname{End}\left(H_{0}\right)$ for all $\beta \in Q_{+}^{\vee}$. Note that $F_{\beta}^{\mu} \equiv 0$ for all but finitely many $\beta \in Q_{+}$.

We first show that $F_{\beta}^{\mu}(\gamma)$ is regular at $\gamma \in T \backslash \mathcal{S}_{+}$. By (5.5) and by the cocycle property, $F_{\beta}^{\mu}(\gamma)$ is regular at $\gamma=\zeta$ if $C_{\left(e, \varpi_{j}\right)}\left(\cdot, q^{\nu} \gamma\right) \in \mathbb{C}[T] \otimes \operatorname{End}\left(H_{0}\right)$ is regular at $\gamma=\zeta$ for all $1 \leq j \leq N$ and $\nu \in \Lambda$. The latter statement follows from the fact that $R_{i}(z)$ has only a (simple) pole at $z=k^{-2}$ and from the explicit expression

$$
\begin{aligned}
C_{\left(e, \varpi_{j}\right)}(t, \gamma)= & C_{\iota}\left(\eta(\pi)\left(t^{-1}\right)\right)^{j}\left(R_{N-j}\left(q \gamma_{1} / \gamma_{N}\right) \cdots R_{2}\left(q \gamma_{1} / \gamma_{j+2}\right) R_{1}\left(q \gamma_{1} / \gamma_{j+1}\right)\right) \\
\times \cdots & \times\left(R_{N-2}\left(q \gamma_{j-1} / \gamma_{N}\right) \cdots R_{j}\left(q \gamma_{j-1} / \gamma_{j+2}\right) R_{j-1}\left(q \gamma_{j-1} / \gamma_{j+1}\right)\right) \\
& \times\left(R_{N-1}\left(q \gamma_{j} / \gamma_{N}\right) \cdots R_{j+1}\left(q \gamma_{j} / \gamma_{j+2}\right) R_{j}\left(q \gamma_{j} / \gamma_{j+1}\right)\right) C_{\iota}
\end{aligned}
$$

which follows from Lemma 4.5(ii) and the cocycle property of $C$.

Let $U \subset T \backslash \mathcal{S}_{+}$be a relatively compact open subset. Choose $\mu \in \Lambda$ such that the closure of $q^{\mu} U$ is contained in $B_{\epsilon}$, where $q^{\mu} U:=\left\{q^{\mu} \gamma \mid \gamma \in U\right\}$. As meromorphic $H_{0}$-valued function in $(t, \gamma) \in B_{\epsilon}^{-1} \times U$, we have

$$
\begin{aligned}
\Psi(t, \gamma) & =D_{(e, \mu)}(t, \gamma) \Psi\left(t, q^{\mu} \gamma\right) \\
& =\sum_{\alpha, \beta \in Q_{+}} F_{\beta}^{\mu}(\gamma)\left(\Gamma_{\alpha}\left(q^{\mu} \gamma\right)\right) t^{-\alpha-\beta} \\
& =\sum_{\alpha \in Q_{+}}\left(\sum_{\beta \in Q_{+}: \alpha-\beta \in Q_{+}} F_{\beta}^{\mu}(\gamma)\left(\Gamma_{\alpha-\beta}\left(q^{\mu} \gamma\right)\right)\right) t^{-\alpha},
\end{aligned}
$$

with the sums converging normally on compacta of $B_{\epsilon}^{-1} \times U$ (note that the sums over $\beta$ are finite). It follows that $\Gamma_{\alpha}\left(\alpha \in Q_{+}\right)$has a unique $H_{0}$-valued meromorphic extension to $T$ which, on $U$, is given by

$$
\Gamma_{\alpha}(\gamma)=\sum_{\beta \in Q_{+}: \alpha-\beta \in Q_{+}} F_{\beta}^{\mu}(\gamma)\left(\Gamma_{\alpha-\beta}\left(q^{\mu} \gamma\right)\right)
$$

such that $\Psi$ on $B_{\epsilon}^{-1} \times U$ admits the power series expansion

$$
\Psi(t, \gamma)=\sum_{\alpha \in Q_{+}} \Gamma_{\alpha}(\gamma) t^{-\alpha}
$$

which converges normally on compacta of $B_{\epsilon}^{-1} \times U$. It follows from (5.14) and the previous paragraph that $\Gamma_{\alpha}$ is analytic on $T \backslash \mathcal{S}_{+}$.

The arguments from the proof of Lemma 5.7, applied to both torus variables of $\Psi(t, \gamma)$ at the same time, directly lead to the following result. 
Proposition 5.8. The $H_{0}$-valued meromorphic function $\Psi(t, \gamma)$ is analytic at $(t, \gamma) \in$ $T \backslash \mathcal{S}_{+}^{-1} \times T \backslash \mathcal{S}_{+}$.

For specialized spectral parameter, we obtain the following result.

Proposition 5.9. Let $\zeta \in T \backslash \mathcal{S}_{+}$.

(i) The $H_{0}$-valued meromorphic function $\Psi(t, \gamma)$ in $(t, \gamma) \in T \times T$ can be specialized at $\gamma=\zeta$, giving rise to a meromorphic $H_{0}$-valued function $\Psi(t, \zeta)$ in $t \in T$. It has the power series expansion

$$
\Psi(t, \zeta)=\sum_{\alpha \in Q_{+}} \Gamma_{\alpha}(\zeta) t^{-\alpha}
$$

for $t \in B_{\epsilon}^{-1}$, normally converging on compacta of $B_{\epsilon}^{-1}$.

(ii) $\Psi(t, \zeta)$ satisfies the gauged $q$-difference equations

$$
D_{(\lambda, e)}(t, \zeta) \Psi\left(q^{-\lambda} t, \zeta\right)=\Psi(t, \zeta), \quad \forall \lambda \in \mathbb{Z}^{N} .
$$

Proof. (i) Restricting to $t \in B_{\epsilon}^{-1}$ for $\epsilon>0$ small enough, the statement is correct by Lemma 5.7. If $t^{\prime} \in T$ is arbitrary then there exists a $\lambda \in \Lambda$ such that $q^{-\lambda} t^{\prime} \in B_{\epsilon}^{-1}$. For $t \in T$ in a small neighborhood of $t^{\prime}$ we then have

$$
\Psi(t, \gamma)=D_{(\lambda, e)}(t, \gamma) \Psi\left(q^{-\lambda} t, \gamma\right) \text {. }
$$

Since $D_{(\lambda, e)} \in\left(Q_{0}(\mathcal{A}) \otimes \mathcal{B}\right) \otimes \operatorname{End}\left(H_{0}\right)$ by Lemma 5.2 (ii) the statement now follows in a small open neighborhood of $t^{\prime}$.

(ii) Specializing the gauged $q$-difference equations $D_{(\lambda, e)}(t, \gamma) \Psi\left(q^{-\lambda} t, \gamma\right)=\Psi(t, \gamma)$ $\left(\lambda \in \mathbb{Z}^{N}\right)$ to $\gamma=\zeta$ yields the desired result.

5.5. Evaluation formula. We write $(z ; q)_{\infty}=\prod_{m=0}^{\infty}\left(1-q^{m} z\right)$ for the $q$-shifted factorial. Recall the power series expansion $\Psi(t, \gamma)=\sum_{\alpha \in Q_{+}} \Gamma_{\alpha}(\gamma) t^{-\alpha}$ for $\left|t^{\alpha_{i}}\right| \gg$ $0(1 \leq i<N)$ from Subsection 5.4 We call the following result the evaluation formula for the basic asymptotically free solution $\Phi_{\kappa}=W_{\kappa} \Psi$ of BqKZ, since it implies the celebrated evaluation formula for the Macdonald polynomials (see Subsection 7.4).

Theorem 5.10. We have

$$
\Gamma_{0}(\gamma)=K(\gamma) T_{w_{0}}
$$

with $K \in \mathcal{M}(T)$ explicitly given by

$$
K(\gamma):=\prod_{1 \leq i<j \leq N} \frac{\left(q \gamma_{i} / \gamma_{j} ; q\right)_{\infty}}{\left(q k^{2} \gamma_{i} / \gamma_{j} ; q\right)_{\infty}} .
$$

Proof. We use the notations of Lemma 5.2 Recall that $\Psi$ satisfies the gauged $q$-difference equations

$$
A_{i}(t, \gamma) \Psi\left(q^{-\varpi_{i}} t, \gamma\right)=\Psi(t, \gamma)
$$

for $1 \leq i \leq N$. In view of the proof of Lemma 5.2 and Lemma 5.7 it reduces in the limit $\left|t^{-\alpha_{i}}\right| \rightarrow 0(1 \leq i<N)$ to

$$
\gamma^{-w_{0}\left(\varpi_{i}\right)} \eta\left(T_{w_{0}} Y^{w_{0}\left(\varpi_{i}\right)} T_{w_{0}}^{-1}\right)(\gamma) \Gamma_{0}(\gamma)=\Gamma_{0}(\gamma)
$$

for $1 \leq i \leq N$, as $H_{0}$-valued meromorphic functions in $\gamma \in T$. This forces $\Gamma_{0}(\gamma)=$ $K(\gamma) \eta\left(T_{w_{0}}\right) \xi_{e}(\gamma)=K(\gamma) T_{w_{0}}$ for some $K \in \mathcal{M}(T)$; see Lemma 4.2 .

It remains to show that $K$ is explicitly given by (5.16). Write $L(\gamma)$ for the right hand side of (5.16). Then $L \in \mathcal{M}(T)$ is characterized by the following three properties: 
(i) for some $\epsilon>0$ we have a power series expansion

$$
L(\gamma)=\sum_{\alpha \in Q_{+}} l_{\alpha} \gamma^{\alpha}
$$

for $\gamma \in B_{\epsilon}$, converging normally on compacta of $B_{\epsilon}$;

(ii) $l_{0}=1$; and

(iii) $L(\gamma)$ satisfies the $q$-difference equations

$$
\prod_{\substack{1 \leq r \leq j \\ j+1 \leq s \leq N}} \frac{1-q \gamma_{r} / \gamma_{s}}{1-q k^{2} \gamma_{r} / \gamma_{s}} L\left(q^{\varpi_{j}} \gamma\right)=L(\gamma), \quad 1 \leq j \leq N .
$$

It thus suffices to show that $K(\gamma)$ satisfies the three properties (i)-(iii). It is clear that $K \in \mathcal{M}(T)$ satisfies (i); see Subsection 5.4 Theorem 5.3(ii) implies (ii) for $K$. What remains is the verification of the $q$-difference equations (iii) for $K$. Using the notations of Lemma 5.2, we write

$$
B_{j}^{(0)}:=\left.B_{j}\right|_{x^{-\alpha_{1}}=0, \ldots, x^{-\alpha_{N-1}=0}} \in Q_{0}(\mathcal{B}) \otimes \operatorname{End}\left(H_{0}\right) .
$$

We view $B_{j}^{(0)}(\gamma)$ as an $\operatorname{End}\left(H_{0}\right)$-valued meromorphic function in $\gamma \in T$. Taking the limit $\left|t^{-\alpha_{i}}\right| \rightarrow 0(1 \leq i<N)$ in the gauged $q$-difference equations

$$
B_{j}(t, \gamma) \Psi\left(t, q^{\varpi_{j}} \gamma\right)=\Psi(t, \gamma), \quad 1 \leq j \leq N
$$

and using $\Gamma_{0}(\gamma)=K(\gamma) T_{w_{0}}$ we obtain

$$
K\left(q^{\varpi_{j}} \gamma\right) B_{j}^{(0)}(\gamma) T_{w_{0}}=K(\gamma) T_{w_{0}}
$$

for $1 \leq j \leq N$, as meromorphic $H_{0}$-valued functions in $\gamma \in T$. Writing $B_{j}^{(0)}(\gamma) T_{w_{0}}=$ $\sum_{w \in S_{N}} a_{w}^{j}(\gamma) T_{w}$ with $a_{w}^{j} \in \mathcal{M}(T)$ it thus suffices to show that

$$
a_{w_{0}}^{j}(\gamma)=\prod_{\substack{1 \leq r \leq j \\ j+1 \leq s \leq N}} \frac{1-q \gamma_{r} / \gamma_{s}}{1-q k^{2} \gamma_{r} / \gamma_{s}}=k^{-\left\langle\delta, \varpi_{j}\right\rangle} \prod_{\substack{1 \leq r \leq j \\ j+1 \leq s \leq N}} c_{k}\left(q \gamma_{r} / \gamma_{s}\right)^{-1}
$$

for $1 \leq j \leq N$, where the second equality follows from a direct computation using the explicit expression (2.5) of $c_{k}$.

By (5.5) we have

$$
B_{j}(t, \gamma)=k^{-\left\langle\delta, \varpi_{j}\right\rangle} t^{w_{0}\left(\varpi_{j}\right)} C_{\left(e, \varpi_{j}\right)}(t, \gamma)
$$

and $C_{\left(e, \varpi_{j}\right)}(t, \gamma)$ is given explicitly by (5.13). Since $R_{i}(z)=c_{k}(z)^{-1}\left(\eta\left(T_{i}\right)-k\right)+1$, Lemma 4.1 and the reduced expression (4.8) for $\sigma^{i}$ imply that

$$
B_{j}(t, \gamma) T_{w_{0}}=k^{-\left\langle\delta, \varpi_{j}\right\rangle} t^{w_{0}\left(\varpi_{j}\right)} C_{\iota}\left(\eta(\pi)\left(t^{-1}\right)\right)^{j}\left(\sum_{w \leq \sigma^{-j}} b_{w}^{j}(\gamma) T_{w w_{0}}\right)
$$

with $\leq$ the Bruhat order on $S_{N}$ and with

$$
b_{\sigma^{-j}}^{j}(\gamma)=\prod_{\substack{1 \leq r \leq j \\ j+1 \leq s \leq N}} c_{k}\left(q \gamma_{r} / \gamma_{s}\right)^{-1} .
$$

By Lemma 4.1 we have

$$
t^{w_{0}\left(\varpi_{j}\right)} C_{\iota}\left(\eta(\pi)\left(t^{-1}\right)\right)^{j} T_{w w_{0}}=t^{w_{0}\left(\varpi_{j}\right)-w_{0} w^{-1} w_{0}\left(\varpi_{j}\right)} T_{w_{0} w^{-1} \sigma^{-j}} .
$$

Hence

$$
B_{j}^{(0)}(\gamma) T_{w_{0}}=k^{-\left\langle\delta, \varpi_{j}\right\rangle} \sum_{w} b_{w}^{j}(\gamma) T_{w_{0} w^{-1} \sigma^{-j}}
$$


with the sum running over $w \in S_{N}$ satisfying $w \leq \sigma^{-j}$ and $w\left(\varpi_{j}\right)=w_{0}\left(\varpi_{j}\right)$. In particular, $a_{w_{0}}^{j}(\gamma)=k^{-\left\langle\delta, \varpi_{j}\right\rangle} b_{\sigma^{-j}}^{j}(\gamma)$. This completes the proof of (5.17).

5.6. Consistency of the bispectral quantum $\mathrm{KZ}$ equations. In this subsection, we show that $\mathrm{BqKZ}$ is a consistent system of $q$-difference equations, i.e., $\operatorname{dim}_{\mathbb{F}}(\mathrm{SOL})=\operatorname{dim}_{\mathbb{C}}\left(H_{0}\right)$, by explicitly constructing an $\mathbb{F}$-basis of SOL. Since the $q$-connection matrices $C_{(\lambda, \mu)}(t, \gamma)\left(\lambda, \mu \in \mathbb{Z}^{N}\right)$ depend rationally on $(t, \gamma) \in T \times T$, the consistency of $\mathrm{BqKZ}$ follows also from the abstract arguments in [13, §5].

We start with a preliminary lemma on the cocycle values $C_{(e, w)}$ for $w \in S_{N}$.

Lemma 5.11. Let $w \in S_{N}$. We have $C_{(e, w)} \in Q_{0}(\mathcal{B}) \otimes \operatorname{End}\left(H_{0}\right)$ and

$$
C_{(e, w)}^{(0)}(h)=k^{-\ell(w)} h T_{w^{-1}}, \quad h \in H_{0},
$$

where

$$
C_{(e, w)}^{(0)}=\left.C_{(e, w)}\right|_{y^{\alpha_{1}}=0, \ldots, y^{\alpha_{N-1}}=0} \in \operatorname{End}\left(H_{0}\right) .
$$

Proof. Let $w=s_{i_{1}} s_{i_{2}} \cdots s_{i_{r}}$ be a reduced expression for $w \in S_{N}\left(1 \leq i_{j}<N\right)$ and write $\beta_{j}:=s_{i_{1}} \cdots s_{i_{j-1}}\left(\alpha_{i_{j}}\right) \in R_{+}$for $1 \leq j \leq r$, where $\beta_{1}$ should be read as $\alpha_{i_{1}}$. By Subsection 4.2 and the cocycle property, we have

$$
C_{(e, w)}(t, \gamma)=C_{\iota} C_{(w, e)}\left(\gamma^{-1}, t^{-1}\right) C_{\iota}=C_{\iota}\left(R_{i_{r}}\left(\gamma^{\beta_{r}}\right) \cdots R_{i_{2}}\left(\gamma^{\beta_{2}}\right) R_{i_{1}}\left(\gamma^{\beta_{1}}\right)\right)^{-1} C_{\iota} .
$$

From the expression for $R_{i}(z)$ it now follows that $C_{(e, w)} \in Q_{0}(\mathcal{B}) \otimes \operatorname{End}\left(H_{0}\right)$. Since $\lim _{z \rightarrow 0} R_{i}(z)=k \eta\left(T_{i}^{-1}\right)$ we furthermore have

$$
C_{(e, w)}^{(0)}=k^{-\ell(w)} C_{\iota} \eta\left(T_{w}\right) C_{\iota} .
$$

The map $C_{\iota}$ is the $\mathbb{K}$-linear extension of the anti-algebra involution of $H_{0}$ mapping $T_{w}$ to $T_{w^{-1}}$. Hence $C_{(e, w)}^{(0)}(h)=k^{-\ell(w)} h T_{w^{-1}}$ for $h \in H_{0}$.

Define $U \in \operatorname{End}\left(H_{0}\right)^{\mathbb{K}}:=\mathbb{K} \otimes \operatorname{End}\left(H_{0}\right)$ by

$$
U\left(k^{-\ell(w)} T_{w_{0}} T_{w^{-1}}\right)=\tau(e, w) \Phi_{\kappa}, \quad w \in S_{N} .
$$

Since SOL is $\mathbb{S}_{N}$-invariant, $U$ is an $\operatorname{End}\left(H_{0}\right)$-valued solution of the BqKZ, i.e.

$$
C_{(\lambda, \mu)}(t, \gamma) U\left(q^{-\lambda} t, q^{\mu} \gamma\right)=U(t, \gamma), \quad \lambda, \mu \in \mathbb{Z}^{N}
$$

as $\operatorname{End}\left(H_{0}\right)$-valued meromorphic functions in $(t, \gamma) \in T \times T$.

Lemma 5.12. $U \in \operatorname{End}\left(H_{0}\right)^{\mathbb{K}}$ is invertible.

Proof. Using the natural identification $\operatorname{End}\left(H_{0}\right)^{\mathbb{K}} \simeq \operatorname{End}_{\mathbb{K}}\left(H_{0}^{\mathbb{K}}\right)$ as $\mathbb{K}$-algebras, we need to verify that $U \in \mathrm{GL}_{\mathbb{K}}\left(H_{0}^{\mathbb{K}}\right)$.

Set $\Phi_{w}:=\tau(e, w) \Phi_{\kappa}$ and $\Psi_{w}:=\tau(e, w) \Psi$ for $w \in S_{N}$, so that $\Phi_{w}(t, \gamma)=$ $W_{\kappa}\left(t, w^{-1} \gamma\right) \Psi_{w}(t, \gamma)$. Since $C_{(e, w)}(t, \gamma)$ is independent of $t \in T$, we simply write it as $C_{(e, w)}(\gamma)$. Recall the $W$-invariant subset $\mathcal{S} \subset T$ (see (4.6)), which contains $\mathcal{S}_{+}$. By Lemma 4.4 and Lemma 5.7, we have for some $\epsilon>0$ the power series expansion

$$
\Psi_{w}(t, \gamma)=\sum_{\alpha \in Q_{+}} C_{(e, w)}(\gamma)\left(\Gamma_{\alpha}\left(w^{-1} \gamma\right)\right) t^{-\alpha}
$$

for $(t, \gamma) \in B_{\epsilon}^{-1} \times T \backslash \mathcal{S}$, converging normally on compacta of $B_{\epsilon}^{-1} \times T \backslash \mathcal{S}$. We write $\Gamma_{\alpha}^{w}(\gamma):=C_{(e, w)}(\gamma)\left(\Gamma_{\alpha}\left(w^{-1} \gamma\right)\right)$ in the remainder of the proof. It is a meromorphic function in $\gamma \in T$, analytic on $T \backslash \mathcal{S}$, and the power series expansion of $\Psi_{w}$ becomes

$$
\Psi_{w}(t, \gamma)=\sum_{\alpha \in Q_{+}} \Gamma_{\alpha}^{w}(\gamma) t^{-\alpha}
$$


Observe that

$$
\Gamma_{0}^{w}(\gamma) \rightarrow C_{(e, w)}^{(0)}\left(T_{w_{0}}\right)=k^{-\ell(w)} T_{w_{0}} T_{w^{-1}},
$$

in the limit $\gamma^{\alpha_{i}} \rightarrow 0(1 \leq i<N)$, in view of the previous lemma.

Write $U=V \Xi$ with $V, \Xi$ the $\mathbb{K}$-linear endomorphisms of $H_{0}^{\mathbb{K}}$ given by

$$
\begin{aligned}
\Xi\left(k^{-\ell(w)} T_{w_{0}} T_{w^{-1}}\right)(t, \gamma) & =W_{\kappa}\left(t, w^{-1} \gamma\right) k^{-\ell(w)} T_{w_{0}} T_{w^{-1}}, \\
V\left(k^{-\ell(w)} T_{w_{0}} T_{w^{-1}}\right) & =\Psi_{w},
\end{aligned}
$$

for $w \in S_{N}$. Since $\Xi \in \mathrm{GL}_{\mathbb{K}}\left(H_{0}^{\mathbb{K}}\right)$ it suffices to show that $V \in \mathrm{GL}_{\mathbb{K}}\left(H_{0}^{\mathbb{K}}\right)$. Let $M$ be the matrix of $V$ with respect to the $\mathbb{K}$-basis $k^{-\ell(w)} T_{w_{0}} T_{w^{-1}}\left(w \in S_{N}\right)$ of $H_{0}^{\mathbb{K}}$. Now fix $\zeta \in T \backslash \mathcal{S}$ such that $\zeta^{\alpha} \notin q^{\mathbb{Z}}$ for all $\alpha \in R$. The matrix $M(t, \gamma)$ may be specialized at $\gamma=\zeta$ and the limit of $M(t, \zeta)$ as $t^{-\alpha_{i}} \rightarrow 0(1 \leq i<N)$ exists. We write $M^{(0)}(\zeta)$ for the limit and $V^{(0)}(\zeta)$ for the corresponding linear endomorphism of $H_{0}$. We then have

$$
V^{(0)}(\zeta)\left(k^{-\ell(w)} T_{w_{0}} T_{w^{-1}}\right)=\Gamma_{0}^{w}(\zeta)=K\left(w^{-1} \zeta\right) C_{(e, w)}(\zeta) T_{w_{0}}
$$

with $K(\gamma)$ given by (5.16). Note that $K\left(w^{-1} \zeta\right) \neq 0$ since $\zeta^{\alpha} \notin q^{\mathbb{Z}}$ for all $\alpha \in R$. By the explicit expression for the cocycle value $C_{(e, w)}(\zeta) \in \operatorname{End}\left(H_{0}\right)$ (see the proof of the previous lemma) we have

$$
C_{(e, w)}(\zeta)\left(T_{w_{0}}\right)=\sum_{v \leq w} a_{v}^{w}(\zeta) T_{w_{0}} T_{v^{-1}},
$$

with $a_{w}^{w}(\zeta) \neq 0$ and with $\leq$ the Bruhat order on $S_{N}$. This implies that $V^{(0)}(\zeta)$ is a linear automorphism of $H_{0}$, hence $\operatorname{det}\left(M^{(0)}(\zeta)\right) \neq 0$. Consequently, $\operatorname{det}(M) \in \mathbb{K}^{\times}$ and $V \in \mathrm{GL}_{\mathbb{K}}\left(H_{0}^{\mathbb{K}}\right)$.

Proposition 5.13. (i) $U^{\prime} \in \operatorname{End}\left(H_{0}\right)^{\mathbb{K}}$ is an $\operatorname{End}\left(H_{0}\right)$-valued meromorphic solution of $B q K Z$ if and only if $U^{\prime}=U F$ for some $F \in \operatorname{End}\left(H_{0}\right)^{\mathbb{F}}$.

(ii) $U$, viewed as $\mathbb{K}$-linear endomorphism of $H_{0}^{\mathbb{K}}$, restricts to an $\mathbb{F}$-linear isomorphism $U: H_{0}^{\mathbb{F}} \rightarrow \mathrm{SOL}$.

(iii) $\left\{\tau(e, w) \Phi_{\kappa}\right\}_{w \in S_{N}}$ is an $\mathbb{F}$-basis of SOL.

Proof. (i) If $U^{\prime}$ is an $\operatorname{End}\left(H_{0}\right)$-valued meromorphic solution of BqKZ then, since $U$ is invertible, we have for all $\lambda, \mu \in \mathbb{Z}^{N}$,

$$
U\left(q^{-\lambda} t, q^{\mu} \gamma\right)^{-1} U^{\prime}\left(q^{-\lambda} t, q^{\mu} \gamma\right)=U(t, \gamma)^{-1} U^{\prime}(t, \gamma)
$$

Hence $U^{\prime}=U F$ with $F \in \operatorname{End}\left(H_{0}\right)^{\mathbb{F}}$. The converse implication is clear.

(ii) By the previous lemma we have $U: H_{0}^{\mathbb{F}} \hookrightarrow$ SOL. It is surjective, since for $g \in \mathrm{SOL}, f:=U^{-1} g \in H_{0}^{\mathbb{K}}$ satisfies $f\left(q^{-\lambda} t, q^{\mu} \gamma\right)=f(t, \gamma)$ for all $\lambda, \mu \in \mathbb{Z}^{N}$ (cf. the proof of (i)), hence $f \in H_{0}^{\mathbb{F}}$.

(iii) This is clear from (ii) and from the definition of $U$.

By Proposition 5.9 and by the proofs of Lemma 5.12 and Proposition 5.13 we obtain the following consistency statement for the quantum KZ equation (4.10) with specialized central character (see [7] and [9]). Recall the $W$-invariant subset $\mathcal{S} \subset T$ given by (4.6). Recall furthermore that $C_{(e, w)}(t, \gamma)$ for $w \in S_{N}$ only depends on $\gamma$, so we simply write it as $C_{(e, w)}(\gamma)$.

Corollary 5.14. Fix $\zeta \in T \backslash \mathcal{S}$ such that $\zeta^{\alpha} \notin q^{\mathbb{Z}}$ for all $\alpha \in R$. For generic $\kappa \in \mathbb{C}^{\times}$, the $H_{0}$-valued meromorphic functions $\left(\tau(e, w) \Phi_{\kappa}\right)(t, \gamma)$ in $(t, \gamma) \in T \times T$ $\left(w \in S_{N}\right)$ can be specialized at $\gamma=\zeta$, giving rise to 
(i) a basis $\left\{C_{(e, w)}(\zeta) \Phi_{\kappa}\left(\cdot, w^{-1} \zeta\right)\right\}_{w \in S_{N}}$ of $\operatorname{SOL}_{\zeta}$ over $\mathcal{E}(T)$;

(ii) an invertible $\operatorname{End}\left(H_{0}\right)$-valued meromorphic solution $U_{\zeta}$ of the quantum $K Z$ equations (4.10), where $U_{\zeta} \in \operatorname{End}\left(H_{0}\right)^{\mathcal{M}(T)}$ is explicitly defined by

$$
U_{\zeta}\left(k^{-\ell(w)} T_{w_{0}} T_{w^{-1}}\right):=C_{(e, w)}(\zeta) \Phi_{\kappa}\left(\cdot, w^{-1} \zeta\right), \quad w \in S_{N} .
$$

\section{The CORRESPONDENCE WITH BISPECTRAL PROBLEMS}

For a finite dimensional affine Hecke algebra module $M$, Cherednik [7, Thm. $4.2]$ derived, for arbitrary root systems, a correspondence between solutions of the quantum affine $\mathrm{KZ}$ equations associated to $M$ and solutions of a system of (possibly matrix-valued) $q$-difference equations. This correspondence was considered before in [6, Thm. 3.4] for a special class of modules $M$, in which case the corresponding $q$-difference operators contain the Macdonald $q$-difference operator (the latter observation is due to Cherednik [6, Thm. 4.4] for $\mathrm{GL}_{N}$, and due to Kato [26, Thm. 4.6] and Cherednik [7 for general root systems). In the classical setting $(q=1)$ it goes back to Matsuo [35].

In the present $\mathrm{GL}_{N}$ setting, with $M=M(\zeta)$ the minimal principal series module with specialized central character $\zeta \in T$, these constructions give rise to an explicit map $\chi_{+}$(independent of $\zeta$ ) from the solution space $\mathrm{SOL}_{\zeta}$ of the quantum $\mathrm{KZ}$ equation (4.10) to the solution space of the spectral problem for the commuting Ruijsenaars' [39] trigonometric $q$-difference operators with spectral parameter $\zeta^{-1}$, see [6. Thm. 4.4] (for $\mathrm{GL}_{N}$ the Macdonald $q$-difference operators coincide with the Ruijsenaars' operators). We recall this result in detail in Subsection 6.4.

In this section we investigate the map $\chi_{+}$when applied to solutions of the bispectral extension of the quantum KZ equations; see Subsection 3.2 In order to do so, we need to replace in the correspondence as described in the previous paragraph, the role of $M(\zeta)$ by the infinite dimensional, formal principal series module of $H$ as defined in Subsection 4.1. We then show that the same map $\chi_{+}$gives rise to an embedding of the solution space SOL of BqKZ into the solution space of a bispectral problem for the Ruijsenaars operators. The techniques employed in this section are analogous to the ones for the usual correspondence (see [9, Chpt. 1]). For the convenience of the reader we provide full details of the arguments involved.

We fix $\kappa \in \mathbb{C}^{\times}$throughout Subsections 6.1,6.3.

6.1. The monodromy cocycle. Observe that $F \in \operatorname{End}\left(H_{0}\right)^{\mathbb{K}}$ is a $\operatorname{End}\left(H_{0}\right)$ valued meromorphic solution of $\mathrm{BqKZ}$ if and only if

$$
\tau(\mathrm{w}) F=F, \quad \mathrm{w} \in \mathbb{Z}^{N} \times \mathbb{Z}^{N},
$$

where the $\mathbb{W}$-action $\tau$ on $\operatorname{End}\left(H_{0}\right)^{\mathbb{K}}$ is defined by

$$
(\tau(\mathrm{w}) F)(t, \gamma):=C_{\mathrm{w}}(t, \gamma)(\mathrm{w} F)(t, \gamma)=C_{\mathrm{w}}(t, \gamma) F\left(\mathrm{w}^{-1}(t, \gamma)\right)
$$

for $\mathrm{w} \in \mathbb{W}$ and $F \in \operatorname{End}\left(H_{0}\right)^{\mathbb{K}}$, viewed as identities between $\operatorname{End}\left(H_{0}\right)$-valued meromorphic functions in $(t, \gamma) \in T \times T$.

By Proposition [5.13(i), given an $\operatorname{End}\left(H_{0}\right)$-valued meromorphic solution $F$ of BqKZ, there exists a unique $G \in \operatorname{End}\left(H_{0}\right)^{\mathbb{F}}$ such that $F=U G$. Accordingly, $G$ describes the deviation of $F$ from the fundamental solution $U$ of $\mathrm{BqKZ}$, and therefore can be thought of as a connection matrix. We will consider the special cases when $F$ are the $\operatorname{End}\left(H_{0}\right)$-valued meromorphic solutions $\tau(\mathrm{w}) U(\mathrm{w} \in \mathbb{W})$ of BqKZ. 
For $\mathrm{w} \in \mathbb{W}$ we set

$$
\mathcal{T}_{\mathrm{w}}:=U^{-1}(\tau(\mathrm{w}) U) \in \operatorname{End}\left(H_{0}\right)^{\mathbb{F}},
$$

that is, $\mathcal{T}_{\mathrm{w}}(\mathrm{w} \in \mathbb{W})$ is the unique element of $\operatorname{End}\left(H_{0}\right)^{\mathbb{F}}$ such that

$$
\tau(\mathrm{w}) U=U \mathcal{T}_{\mathrm{w}} .
$$

Note that $\operatorname{End}\left(H_{0}\right)^{\mathbb{F}}$ is a $\mathbb{W}$-stable subalgebra of End $\left(H_{0}\right)^{\mathbb{K}}$ with respect to the action $(\mathrm{w} F)(t, \gamma)=F\left(\mathrm{w}^{-1}(t, \gamma)\right)$. The following lemma now shows that the $\mathcal{T}_{\mathrm{w}}$ $(\mathrm{w} \in \mathbb{W})$ define a cocycle of $\mathbb{W}$ with values in the group of units of $\operatorname{End}\left(H_{0}\right)^{\mathbb{F}}$.

Lemma 6.1. (i) $\mathcal{T}_{\mathrm{w}}=$ id for $\mathrm{w} \in \mathbb{Z}^{N} \times \mathbb{Z}^{N}$.

(ii) For $\mathrm{w}, \mathrm{w}^{\prime} \in \mathbb{W}$ we have the cocycle relation

$$
\mathcal{T}_{\mathrm{ww}^{\prime}}=\mathcal{T}_{\mathrm{w}} \mathrm{w}\left(\mathcal{T}_{\mathrm{w}^{\prime}}\right)
$$

in $\operatorname{End}\left(H_{0}\right)^{\mathbb{F}}$.

Proof. (i) This follows immediately from the fact that $U$ is an $\operatorname{End}\left(H_{0}\right)$-valued meromorphic solution of BqKZ.

(ii) Note that $\mathcal{T}_{\mathrm{w}}=U^{-1} C_{\mathrm{w}} \mathrm{w}(U)$ for $\mathrm{w} \in \mathbb{W}$. By the cocycle condition for $C_{\mathrm{w}} \in$ $\operatorname{End}\left(H_{0}\right)^{\mathbb{K}}$, which reads in the present notations as $C_{\mathrm{ww}^{\prime}}=C_{\mathrm{w}} \mathrm{w}\left(C_{\mathrm{w}^{\prime}}\right)$ for w, $\mathrm{w}^{\prime} \in \mathbb{W}$, we have

$$
\begin{aligned}
\mathcal{T}_{\mathrm{ww}^{\prime}} & =U^{-1} C_{\mathrm{ww}^{\prime}} \mathrm{ww}^{\prime}(U)=U^{-1} C_{\mathrm{w}} \mathrm{w}\left(C_{\mathrm{w}^{\prime}} \mathrm{w}^{\prime}(U)\right) \\
& =U^{-1} C_{\mathrm{w}} \mathrm{w}(U) \mathrm{w}\left(U^{-1} C_{\mathrm{w}^{\prime} \mathrm{w}^{\prime}}(U)\right)=\mathcal{T}_{\mathrm{w}} \mathrm{w}\left(\mathcal{T}_{\mathrm{w}^{\prime}}\right)
\end{aligned}
$$

for all $\mathrm{w}, \mathrm{w}^{\prime} \in \mathbb{W}$.

Definition 6.2. In analogy with the terminology in [9, §1.3.3] for the quantum KZ equation, we call $\left\{\mathcal{T}_{\mathrm{w}}\right\}_{\mathrm{w} \in \mathbb{W}}$ the monodromy cocycle of the BqKZ.

Remark 6.3. Connection matrices and Riemann-Hilbert problems for ordinary linear $q$-difference equations have been extensively studied; see, e.g., [3] and [1]. For quantum KZ equations, connection matrices have been computed explicitly in, e.g., [20], [14, §12], and [27].

6.2. The correspondence. Consider the algebra $\mathbb{C}(T \times T) \# \mathbb{W}$, where $\mathbb{W}$ acts as field automorphisms on $\mathbb{C}(T \times T)$ by the formula 3.1 . Recall that $\mathbb{C}(T \times T) \# \mathbb{W}$ naturally acts on $\mathbb{K}$. We write $D f$ for the action of $D \in \mathbb{C}(T \times T) \# \mathbb{W}$ on $f \in \mathbb{K}$.

We have a representation $\vartheta: \mathbb{C}(T \times T) \# \mathbb{W} \rightarrow \operatorname{End}\left(\operatorname{End}\left(H_{0}\right)^{\mathbb{K}}\right)$ given by

$$
\begin{aligned}
& \vartheta(f) F=f F, \quad f \in \mathbb{C}(T \times T), \\
& \vartheta(\mathrm{w}) F=\mathrm{w}(F), \quad \mathrm{w} \in \mathbb{W}
\end{aligned}
$$

for $F \in \operatorname{End}\left(H_{0}\right)^{\mathbb{K}}$. Let $\mathbb{D}$ be the subalgebra $\mathbb{C}(T \times T) \#\left(\mathbb{Z}^{N} \times \mathbb{Z}^{N}\right)$ of $\mathbb{C}(T \times T) \# \mathbb{W}$. Under the natural action of $\mathbb{C}(T \times T) \# \mathbb{W}$ on $\mathbb{C}(T \times T)$, the subalgebra $\mathbb{D}$ identifies with the algebra of $q$-difference operators on $T \times T$ with rational coefficients.

Set $H_{0}^{*}:=\operatorname{Hom}\left(H_{0}, \mathbb{C}\right)$. We will regard a linear functional $\chi \in H_{0}^{*}$ also as an element of $\operatorname{Hom}_{\mathbb{K}}\left(H_{0}^{\mathbb{K}}, \mathbb{K}\right)$ by $\mathbb{K}$-linear extension. For $F \in \operatorname{End}\left(H_{0}\right)^{\mathbb{K}}$, denote

$$
\phi_{\chi, v}^{F}:=\chi(F v) \in \mathbb{K}, \quad \chi \in H_{0}^{*}, v \in H_{0}
$$

for its matrix coefficients. Note that for any $D \in \mathbb{C}(T \times T) \# \mathbb{W}, \chi \in H_{0}^{*}$ and $v \in H_{0}$,

$$
D \phi_{\chi, v}^{F}=\phi_{\chi, v}^{\vartheta(D) F}
$$

for all $F \in \operatorname{End}\left(H_{0}\right)^{\mathbb{K}}$. 
Lemma 6.4. For $\mathrm{w} \in \mathbb{W}$ we have

$$
\vartheta(\mathrm{w}) U=C_{\mathrm{w}}^{-1} U \mathcal{T}_{\mathrm{w}} .
$$

In particular, $\vartheta(\mathrm{w}) U=C_{\mathrm{w}}^{-1} U$ for $\mathrm{w} \in \mathbb{Z}^{N} \times \mathbb{Z}^{N}$.

Proof. For $\mathrm{w} \in \mathbb{W}$

$$
\vartheta(\mathrm{w}) U=\mathrm{w}(U)=C_{\mathrm{w}}^{-1}(\tau(\mathrm{w}) U)=C_{\mathrm{w}}^{-1} U \mathcal{T}_{\mathrm{w}} .
$$

The second claim follows from the fact that $\mathcal{T}_{\mathrm{w}}=$ id for $\mathrm{w} \in \mathbb{Z}^{N} \times \mathbb{Z}^{N}$.

We now are going to look for a particular linear functional $\chi$ such that the matrix coefficients $\phi_{\chi, v}^{U}\left(v \in H_{0}\right)$ of $U$ solves a bispectral problem with respect to two commuting families of Ruijsenaars' trigonometric $q$-difference operators (one family acting on the first torus component, the second on the second torus component). In view of (6.1) and the previous lemma, to obtain $q$-difference equations for $\phi_{\chi, v}$ we have to deal with the cocycle value $C_{\mathrm{w}}$ and the monodromy matrix $\mathcal{T}_{\mathrm{w}}$ in the equations $\mathrm{w} \phi_{\chi, v}^{U}=\phi_{\chi, v}^{C_{\mathrm{w}}^{-1} U \mathcal{T}_{\mathrm{w}}}$. It is convenient to postpone the analysis of the monodromy cocycle by initially absorbing it into the action $\vartheta$ of $\mathbb{C}(T \times T) \# \mathbb{W}$ via the twisted algebra homomorphism

$$
\vartheta_{\mathcal{T}}: \mathbb{C}(T \times T) \# \mathbb{W} \rightarrow \operatorname{End}\left(\operatorname{End}\left(H_{0}\right)^{\mathbb{K}}\right),
$$

defined by

$$
\begin{array}{ll}
\vartheta_{\mathcal{T}}(f) F=f F, & f \in \mathbb{C}(T \times T), \\
\vartheta_{\mathcal{T}}(\mathrm{w}) F=\mathrm{w}(F) \mathcal{T}_{\mathrm{w}}^{-1}, & \mathrm{w} \in \mathbb{W}
\end{array}
$$

for $F \in \operatorname{End}\left(H_{0}\right)^{\mathbb{K}}$. Note that $\vartheta_{\mathcal{T}}$ is indeed an algebra homomorphism, thanks to the cocycle condition for $\mathcal{T}$. Moreover, $\left.\vartheta_{\mathcal{T}}\right|_{\mathbb{D}}=\left.\vartheta\right|_{\mathbb{D}}$.

For $D \in \mathbb{C}(T \times T) \# \mathbb{W}$ we will occasionally use the notations

$$
D=\sum_{\mathrm{w} \in \mathbb{W}} d_{\mathrm{w}} \mathrm{w}=\sum_{\mathrm{v} \in \mathbb{S}_{N}} D_{\mathrm{v}} \mathrm{v}
$$

where $d_{\mathrm{w}} \in \mathbb{C}(T \times T)(\mathrm{w} \in \mathbb{W})$ and $D_{\mathrm{v}}=\sum_{\mathrm{u} \in \mathbb{Z}^{N} \times \mathbb{Z}^{N}} d_{\mathrm{uv}} \mathrm{u} \in \mathbb{D}\left(\mathrm{v} \in \mathbb{S}_{N}\right)$. Reformulating (6.1) and Lemma 6.4 in terms of the twisted action $\vartheta_{\mathcal{T}}$ yields the following result.

Lemma 6.5. (i) For $\mathrm{w} \in \mathbb{W}$ we have

$$
\vartheta_{\mathcal{T}}(\mathrm{w}) U=C_{\mathrm{w}}^{-1} U
$$

(ii) For $D \in \mathbb{C}(T \times T) \# \mathbb{W}$ we have

$$
\phi_{\chi, v}^{\vartheta \tau(D) U}=\sum_{\mathrm{v} \in \mathbb{S}_{N}} D_{\mathrm{v}}\left(\phi_{\chi, v}^{C_{\mathrm{v}}^{-1} U}\right)
$$

for all $\chi \in H_{0}^{*}$ and $v \in H_{0}$.

Proof. (i) This is clear from Lemma 6.4 and the definition of $\vartheta_{\mathcal{T}}$.

(ii) By Lemma 6.4 and (6.1), we obtain

$$
\phi_{\chi, v}^{\vartheta \tau(D) U}=\sum_{\mathrm{v} \in \mathbb{S}_{N}} \phi_{\chi, v}^{\vartheta\left(D_{\mathrm{v}}\right) \vartheta \mathcal{T}(\mathrm{v}) U}=\sum_{\mathrm{v} \in \mathbb{S}_{N}} D_{\mathrm{v}}\left(\phi_{\chi, v}^{\vartheta \mathcal{\tau}(\mathrm{v}) U}\right)
$$

The result now follows from (i). 
We define the restriction map Res: $\mathbb{C}(T \times T) \# \mathbb{W} \rightarrow \mathbb{D}$ to be the $\mathbb{C}(T \times T)$-linear map

$$
\operatorname{Res}(D):=\sum_{\mathrm{v} \in \mathbb{S}_{N}} D_{\mathrm{v}}, \quad D \in \mathbb{D} .
$$

Lemma 6.4(ii) implies that if we have a linear functional $\chi_{+} \in H_{0}^{*}$ such that $\chi_{+}\left(C_{\mathrm{v}}^{-1} U\right)=\chi_{+}(U)$ for all $\mathrm{v} \in \mathbb{S}_{N}$, then the corresponding matrix coefficients $\phi_{\chi_{+}, v}^{U}\left(v \in H_{0}\right)$ satisfy

$$
\operatorname{Res}(D)\left(\phi_{\chi_{+}, v}^{U}\right)=\phi_{\chi_{+}, v}^{\vartheta_{\mathcal{T}}(D) U}
$$

for all $D \in \mathbb{C}(T \times T) \# \mathbb{W}$.

Lemma 6.6. Define $\chi_{+} \in H_{0}^{*}$ by $\chi_{+}\left(T_{w}\right)=k^{\ell(w)}$ for all $w \in S_{N}$. Then

$$
\chi_{+}\left(C_{\mathrm{v}}^{-1} F\right)=\chi_{+}(F)
$$

for $F \in \operatorname{End}\left(H_{0}\right)^{\mathbb{K}}$ and $\mathrm{v} \in \mathbb{S}_{N}$.

Proof. Since $C_{\iota}\left(T_{w}\right)=T_{w^{-1}}$ for $w \in S_{N}$ we have $\chi_{+} \circ C_{\iota}=\chi_{+}$. By the cocycle condition for $C_{\mathrm{w}}\left(\mathrm{w} \in \mathbb{S}_{N}\right)$ it remains to prove that $\chi_{+} \circ C_{\left(s_{i}, e\right)}=\chi_{+}$for $1 \leq i<N$. But this follows from the expression $C_{\left(s_{i}, e\right)}(t, \gamma)=c_{k}\left(t_{i} / t_{i+1}\right)^{-1}\left(\eta\left(T_{i}\right)-k\right)+1$ (see Lemma 4.3) since

$$
\chi_{+}\left(\left(T_{i}-k\right) h\right)=0
$$

for $1 \leq i<N$ and $h \in H_{0}$.

If $D \in \mathbb{C}(T \times T) \# \mathbb{W}$ satisfies $\vartheta_{\mathcal{T}}(D) U=\lambda U$ for some $\lambda \in \mathbb{K}$, then it follows from (6.3) that the matrix coefficients $\phi_{\chi_{+}, v}^{U}\left(v \in H_{0}\right)$ are eigenfunctions of $\operatorname{Res}(D)$ with eigenvalue $\lambda$. We will now construct such a commuting family of $D$ 's. It leads to the interpretation of the $\phi_{\chi_{+}, v}\left(v \in H_{0}\right)$ as solutions of a bispectral problem.

The appropriate elements $D \in \mathbb{C}(T \times T) \# \mathbb{W}$ are obtained as images of elements from the center $Z(H)$ of the affine Hecke algebra $H$ under the faithful algebra homomorphism $\rho$ from Theorem 2.4. Since we aim at a bispectral version, we will interpret $\rho$ as algebra map $\rho: H \rightarrow \mathbb{C}(T \times T) \# \mathbb{W}$ in two different ways. We have, on the one hand, the algebra homomorphism

$$
\rho_{k^{-1}, q}^{x}: H\left(k^{-1}\right) \rightarrow \mathbb{C}(T \times T) \# \mathbb{W},
$$

which is the map $\rho_{k^{-1}, q}$ from Theorem 2.4, interpreted as algebra homomorphism from $H\left(k^{-1}\right)$ to the subalgebra $\mathbb{C}(T \times\{1\}) \#(W \times\{e\})$ of $\mathbb{C}(T \times T) \# \mathbb{W}$. On the other hand, we have an algebra homomorphism

$$
\rho_{k, q^{-1}}^{y}: H(k) \rightarrow \mathbb{C}(T \times T) \# \mathbb{W},
$$

defined as the map $\rho_{k, q^{-1}}$ from Theorem 2.4 interpreted as algebra homomorphism from $H(k)$ to the subalgebra $\mathbb{C}(\{1\} \times T) \#(\{e\} \times W)$ of $\mathbb{C}(T \times T) \# \mathbb{W}$. Note that they can be combined into an algebra homomorphism

$$
\rho_{k^{-1}, q}^{x} \times \rho_{k, q^{-1}}^{y}: H\left(k^{-1}\right) \otimes H(k) \rightarrow \mathbb{C}(T \times T) \# \mathbb{W} .
$$

Definition 6.7. (i) For $h \in H\left(k^{-1}\right)$, define

$$
D_{h}^{x}:=\rho_{k^{-1}, q}^{x}(h) \in \mathbb{C}(T \times T) \# \mathbb{W} .
$$

(ii) For $h \in H(k)$, define

$$
D_{h}^{y}:=\rho_{k, q^{-1}}^{y}(h) \in \mathbb{C}(T \times T) \# \mathbb{W} .
$$


Remark 6.8. Let ${ }^{\circ}: H\left(k^{-1}\right) \rightarrow H(k)$ be the algebra isomorphism defined by $\pi^{\circ}=\pi$ and $T_{i}^{\circ}=T_{i}^{-1}$ for $1 \leq i<N$. Then

$$
D_{h^{\circ}}^{y}=\iota D_{h}^{x} \iota, \quad \forall h \in H\left(k^{-1}\right) .
$$

This follows by verifying the identity

$$
\rho_{k, q^{-1}}^{y}\left(h^{\circ}\right)=\iota \rho_{k^{-1}, q}^{x}(h) \iota
$$

for the algebraic generators $\pi$ and $T_{i}(1 \leq i<N)$ of $H\left(k^{-1}\right)$ using Theorem 2.4 .

Recall the formal principal series representation, encoded by the algebra homomorphism $\eta: H(k) \rightarrow \operatorname{End}\left(H_{0}\right)^{\mathbb{K}}$ (see Subsection 4.1).

Proposition 6.9. (i) For $h \in H\left(k^{-1}\right)$ we have

$$
\vartheta_{\mathcal{T}}\left(D_{h}^{x}\right) U=\eta\left(h^{\dagger}\right) U
$$

where $\dagger: H\left(k^{-1}\right) \rightarrow H(k)$ is the unique anti-algebra isomorphism satisfying

$$
T_{i}^{\dagger}=T_{i}^{-1}, \quad \pi^{\dagger}=\pi^{-1}
$$

for $1 \leq i<N$.

(ii) For $h \in H(k)$ we have

$$
\vartheta_{\mathcal{T}}\left(D_{h}^{y}\right) U=C_{\iota} \iota\left(\eta\left(h^{\ddagger}\right)\right) C_{\iota} U,
$$

where $\ddagger: H(k) \rightarrow H(k)$ is the unique anti-algebra involution satisfying

$$
T_{i}^{\ddagger}=T_{i}, \quad \pi^{\ddagger}=\pi^{-1}
$$

for $1 \leq i<N$ (note that $\left.\dagger=\ddagger 0^{\circ}\right)$.

Proof. (i) We first show that it suffices to prove (6.6) for algebraic generators of $H\left(k^{-1}\right)$. Indeed, if (6.6) is valid for $h, h^{\prime} \in H\left(k^{-1}\right)$, then we have

$$
\begin{aligned}
\vartheta_{\mathcal{T}}\left(D_{h h^{\prime}}^{x}\right) U & =\vartheta_{\mathcal{T}}\left(D_{h}^{t}\right) \vartheta_{\mathcal{T}}\left(D_{h^{\prime}}^{x}\right) U=\vartheta_{\mathcal{T}}\left(D_{h}^{x}\right) \eta\left(h^{\prime \dagger}\right) U \\
& =\eta\left(h^{\prime \dagger}\right) \vartheta_{\mathcal{T}}\left(D_{h}^{x}\right) U=\eta\left(h^{\prime \dagger}\right) \eta\left(h^{\dagger}\right) U \\
& =\eta\left(\left(h h^{\prime}\right)^{\dagger}\right) U,
\end{aligned}
$$

where the third equality follows since $\left[\vartheta_{\mathcal{T}}\left(D_{h}^{x}\right), \eta\left(h^{\prime \dagger}\right)\right]=0$ as endomorphisms of $\operatorname{End}_{\mathbb{K}}\left(H_{0}^{\mathbb{K}}\right)$ (here $\eta\left(h^{\prime \dagger}\right)$ should be viewed as element in $\operatorname{End}\left(\operatorname{End}\left(H_{0}\right)^{\mathbb{K}}\right)$ by left multiplication). Indeed, since $\eta\left(h^{\prime \dagger}\right)(t, \gamma)$ does not depend on the torus parameter $t \in T$, it commutes with $\vartheta_{\mathcal{T}}\left(D_{h}^{x}\right) \in \vartheta_{\mathcal{T}}(\mathbb{C}(T \times\{1\}) \#(W \times\{e\}))$ (which involves, besides the action of $W \times\{e\}$, only right multiplication by the monodromy cocycle).

So it remains to verify (6.6) for $h=\pi \in H\left(k^{-1}\right)$ and for $h=T_{i} \in H\left(k^{-1}\right)$ $(1 \leq i<N)$. For $h=\pi \in H\left(k^{-1}\right)$ we have

$$
\vartheta_{\mathcal{T}}\left(D_{\pi}^{x}\right) U=\vartheta_{\mathcal{T}}((\pi, e)) U=C_{(\pi, e)}^{-1} U=\eta\left(\pi^{\dagger}\right) U,
$$

where the last equality follows from (4.5). For $h=T_{i} \in H\left(k^{-1}\right)(1 \leq i<N)$, we have

$$
\begin{aligned}
\vartheta_{\mathcal{T}}\left(D_{T_{i}}^{x}\right) U & =\left(k^{-1}-c_{k}\left(x_{i+1} / x_{i}\right)\right) U+c_{k}\left(x_{i+1} / x_{i}\right) \vartheta_{\mathcal{T}}\left(\left(s_{i}, e\right)\right) U \\
& =\left(k^{-1}-c_{k}\left(x_{i+1} / x_{i}\right) U+c_{k}\left(x_{i+1} / x_{i}\right) C_{\left(s_{i}, e\right)}^{-1} U\right. \\
& =\eta\left(T_{i}^{\dagger}\right) U
\end{aligned}
$$

where we used that $c_{k^{-1}}\left(z^{-1}\right)=c_{k}(z)$ in the first equality, while the second equality follows from Lemma 6.5 (i) and the third equality from Lemma 4.3 
(ii) Unfortunately it is not possible to derive (ii) directly from (i) and from (6.5). Instead, one has to repeat the steps of the proof of (i). It again amounts to verifying (6.7) for $h=\pi \in H(k)$ and for $h=T_{i} \in H(k)(1 \leq i<N)$. We show the second case, the first case is left to the reader.

Let $1 \leq i<N$. Then we have for $T_{i} \in H(k)$,

$$
\begin{aligned}
\vartheta_{\mathcal{T}}\left(D_{T_{i}}^{y}\right) U & =\left(k-c_{k}\left(y_{i} / y_{i+1}\right)\right) U+c_{k}\left(y_{i} / y_{i+1}\right) \vartheta_{\mathcal{T}}\left(\left(e, s_{i}\right)\right) U \\
& =\left(k-c_{k}\left(y_{i} / y_{i+1}\right)\right) U+c_{k}\left(y_{i} / y_{i+1}\right) C_{\left(e, s_{i}\right)}^{-1} U .
\end{aligned}
$$

Since

$$
C_{\left(e, s_{i}\right)}=C_{\iota} \iota\left(C_{\left(s_{i}, e\right)}\right) C_{\iota}
$$

by the cocycle condition (recall Remark 3.5) and since $C_{\iota}^{2}=\mathrm{id}$ and $C_{\left(s_{i}, e\right)}(t, \gamma)^{-1}=$ $C_{\left(s_{i}, e\right)}\left(s_{i} t, \gamma\right)$, Lemma 4.3 implies that

$$
C_{\left(e, s_{i}\right)}^{-1}=c_{k}\left(y_{i} / y_{i+1}\right)^{-1}\left(C_{\iota} \iota\left(\eta\left(T_{i}\right)\right) C_{\iota}-k\right)+1 .
$$

Substituting in (6.8) gives $\vartheta_{\mathcal{T}}\left(D_{T_{i}}^{y}\right) U=C_{\iota} \iota\left(\eta\left(T_{i}^{\ddagger}\right)\right) C_{\iota} U$, as desired.

The following lemma plays an important role in the bispectral version of the correspondence. Recall that the center $Z(H)$ of the affine Hecke algebra $H$ is given by $\mathbb{C}_{Y}[T]^{S_{N}}$ (Bernstein, see $[30]$ ).

Lemma 6.10. For $p \in \mathbb{C}[T]^{S_{N}}$ we have

$$
p(Y)^{\dagger}=p\left(Y^{-1}\right), \quad p(Y)^{\ddagger}=p\left(Y^{-1}\right) .
$$

Proof. By (2.4) it immediately follows that $Y_{i}^{\dagger}=Y_{i}^{-1}$ for $1 \leq i \leq N$. This implies the first formula.

For the second formula, it suffices to show that

$$
Y_{i}^{\ddagger}=T_{w_{0}} Y_{N-i+1}^{-1} T_{w_{0}}^{-1}
$$

in $H(k)$ for $1 \leq i \leq N$, since we then have, for $p \in \mathbb{C}[T]^{S_{N}}$,

$$
p(Y)^{\ddagger}=T_{w_{0}} p\left(Y_{N}^{-1}, \ldots, Y_{1}^{-1}\right) T_{w_{0}}^{-1}=T_{w_{0}} p\left(Y^{-1}\right) T_{w_{0}}^{-1}=p\left(Y^{-1}\right),
$$

where the last equality follows from the fact that $p\left(Y^{-1}\right) \in Z(H(k))$. To prove (6.9), note that $T_{w_{0}} T_{i}^{-1}=T_{N-i}^{-1} T_{w_{0}}$, and $Y_{i+1}=T_{i}^{-1} Y_{i} T_{i}^{-1}$ by (2.4), for $1 \leq i<N$. Hence (6.9) holds for $Y_{i+1}$ if it is true for $Y_{i}$. It thus remains to prove (6.9) for $i=1$. We will use the following observation. Write $\sigma_{i}=s_{i} s_{i+1} \cdots s_{N-1}(1 \leq i<N)$ and $\tau_{i}=s_{j} \cdots s_{N-2}(1 \leq j<N-1)$, which are reduced expressions in $\bar{S}_{N}$. Then the longest Weyl group element $w_{0} \in S_{N}$ can be written as

$$
\begin{aligned}
w_{0} & =\sigma_{N-1} \sigma_{N-2} \cdots \sigma_{1} \\
& =\sigma_{1}\left(\tau_{N-2} \tau_{N-3} \cdots \tau_{1}\right),
\end{aligned}
$$

and $\ell\left(w_{0}\right)$ is the sum of the lengths of the factors in the respective products in (6.10).

By (2.4), formula (6.9) for $i=1$ will be valid if

$$
T_{\sigma_{1}} \pi^{-1}=T_{w_{0}} \pi^{-1} T_{\sigma_{1}} T_{w_{0}}^{-1}
$$

in $H(k)$. By the first expression in (6.10) and the fact that $\pi^{-1} T_{i+1} \pi=T_{i}$ for $1 \leq i<N-1$, we have in $H(k)$,

$$
\pi^{-1} T_{\sigma_{1}} T_{w_{0}}^{-1}=\pi^{-1} T_{\sigma_{2}}^{-1} T_{\sigma_{3}}^{-1} \cdots T_{\sigma_{N-1}}^{-1}=T_{\tau_{1}}^{-1} T_{\tau_{2}}^{-1} \cdots T_{\tau_{N-2}}^{-1} \pi^{-1},
$$


so that (6.11) will follow from

$$
T_{\sigma_{1}}=T_{w_{0}} T_{\tau_{1}}^{-1} T_{\tau_{2}}^{-1} \cdots T_{\tau_{N-2}}^{-1}
$$

in $H(k)$. But this is a direct consequence of the second expression of $w_{0}$ in (6.10).

Corollary 6.11. For $p \in \mathbb{C}[T]^{S_{N}}$, we have $p(Y)^{\circ}=p(Y)$, where ${ }^{\circ}: H\left(k^{-1}\right) \rightarrow$ $H(k)$ is the algebra isomorphism defined in Remark 6.8 .

Proof. This follows from the previous lemma and the fact that $\dagger=\ddagger 0^{\circ}$.

Definition 6.12. (i) Define

$$
L_{p}^{x}:=\operatorname{Res}\left(D_{p(Y)}^{x}\right) \in \mathbb{D}, \quad p \in \mathbb{C}[T]^{S_{N}},
$$

where $p(Y)$ is the corresponding element in $\mathbb{C}_{Y}[T]^{S_{N}}=Z\left(H\left(k^{-1}\right)\right)$.

(ii) Define

$$
L_{p}^{y}:=\operatorname{Res}\left(D_{p(Y)}^{y}\right) \in \mathbb{D}, \quad p \in \mathbb{C}[T]^{S_{N}},
$$

where $p(Y)$ is the corresponding element in $Z(H(k))$.

By Corollary 6.11 and (6.5) we have

$$
L_{p}^{y}=\iota L_{p}^{x} \iota, \quad \forall p \in \mathbb{C}[T]^{S_{N}} .
$$

Furthermore, it is well-known (see [9] and 34]) that the $L_{p}^{x} \in \mathbb{C}(T \times\{1\}) \#\left(\mathbb{Z}^{N} \times\right.$ $\{e\}) \subset \mathbb{D}$ are pairwise commuting and $S_{N} \times S_{N}$-invariant,

$$
{ }_{\mathrm{w}} L_{p}^{x} \mathrm{w}^{-1}=L_{p}^{x}, \quad \forall \mathrm{w} \in S_{N} \times S_{N} .
$$

Similarly, the $L_{p}^{y}=\iota L_{p}^{x} \iota \in \mathbb{C}(\{1\} \times T) \#\left(\{e\} \times \mathbb{Z}^{N}\right) \subset \mathbb{D}$ are pairwise commuting and $S_{N} \times S_{N}$-invariant. Clearly also $\left[L_{p}^{x}, L_{p^{\prime}}^{y}\right]=0$ for all $p, p^{\prime} \in \mathbb{C}[T]^{S_{N}}$ in $\mathbb{D}$.

For the elementary symmetric functions $e_{i} \in \mathbb{C}[T]^{S_{N}}(1 \leq i \leq N)$ given by

$$
e_{i}(t)=\sum_{\substack{I \subseteq\{1, \ldots, N\} \\ \# I=i}} \prod_{j \in I} t_{j}
$$

the corresponding $L_{e_{i}}^{x}$, viewed as elements in

$$
\mathbb{C}(T) \#_{q} \mathbb{Z}^{N} \simeq \mathbb{C}(T \times\{1\}) \#\left(\mathbb{Z}^{N} \times\{e\}\right) \subset \mathbb{D},
$$

are explicitly given by

$$
L_{e_{i}}^{x}=\sum_{\substack{I \subseteq\{1, \ldots, N\} \\ \# I=i}}\left(\prod_{\substack{r \in I \\ s \notin I}} \frac{k x_{r}-k^{-1} x_{s}}{x_{r}-x_{s}}\right) \sum_{r \in I} \epsilon_{r} \in \mathbb{C}(T) \#_{q} \mathbb{Z}^{N}, \quad 1 \leq i \leq N
$$

see, e.g., [9, §1.3.5] and [28. Hence, the $L_{e_{i}}^{x}(1 \leq i \leq N)$ are, under their natural interpretation as $q$-difference operators on $\mathcal{M}(T)$, Ruijsenaars' commuting, trigonometric $q$-difference operators from [39].

Definition 6.13. Consider the bispectral problem

$$
\begin{aligned}
& \left(L_{p}^{x} f\right)(t, \gamma)=p\left(\gamma^{-1}\right) f(t, \gamma), \quad \forall p \in \mathbb{C}[T]^{S_{N}}, \\
& \left(L_{p}^{y} f\right)(t, \gamma)=p(t) f(t, \gamma), \quad \forall p \in \mathbb{C}[T]^{S_{N}}
\end{aligned}
$$

for $f \in \mathbb{K}$, where the equations (6.13) are viewed as identities between meromorphic functions in $(t, \gamma) \in T \times T$. We write BiSP $\subset \mathbb{K}$ for the set of solutions $f \in \mathbb{K}$ of (6.13). 
Remark 6.14. The bispectral problem for ordinary linear differential operators was introduced by Duistermaat and Grünbaum in [12. Many different types of bispectral problems have since been considered. In particular, in [21 the bispectral problem for ordinary linear second-order $q$-difference operators is investigated. For $N=2$, our bispectral problem belongs to this class.

The preceding remarks on the invariance properties of the $L_{p}^{x}$ and the $L_{p}^{y}(p \in$ $\mathbb{C}[T]^{S_{N}}$ ) directly give

Lemma 6.15. BiSP is an $\mathbb{S}_{N}$-invariant $\mathbb{F}$-subspace of $\mathbb{K}$ with respect to the usual $\mathbb{S}_{N}$-action $(\mathrm{w} f)(t, \gamma)=f\left(\mathrm{w}^{-1}(t, \gamma)\right)$ on $f \in \mathbb{K}$.

We can now prove the following bispectral version of the correspondence between solutions of the quantum KZ equations and the spectral problem of the $L_{p}^{x}$ ( $p \in$ $\left.\mathbb{C}[T]^{S_{N}}\right)$.

Theorem 6.16. The linear functional $\chi_{+} \in H_{0}^{*}$ (see Lemma 6.6) defines a $\mathbb{S}_{N^{-}}$ equivariant $\mathbb{F}$-linear map

$$
\chi_{+}: \mathrm{SOL} \rightarrow \mathrm{BiSP} .
$$

Proof. The $\mathbb{K}$-linear extended linear functional $\chi_{+}$defines an $\mathbb{S}_{N}$-equivariant, $\mathbb{F}$ linear map $\chi_{+}: H_{0}^{\mathbb{K}} \rightarrow \mathbb{K}$, since Lemma 6.6 implies that $\chi_{+}(\tau(\mathrm{w}) f)=\mathrm{w}\left(\chi_{+} f\right)$ for w $\in \mathbb{S}_{N}$ and $f \in H_{0}^{\mathbb{K}}$. Hence $\chi_{+}$restricts to an $\mathbb{S}_{N}$-equivariant, $\mathbb{F}$-linear map $\chi_{+}: \mathrm{SOL} \rightarrow \mathbb{K}$.

It remains to show that $\chi_{+}(f) \in \operatorname{BiSP}$ if $f \in \mathrm{SOL}$. Let $f \in \mathrm{SOL}$. By Proposition 5.13 and $\mathbb{F}$-linearity, it suffices only to consider $f$ of the form $f=U v$ for $v \in H_{0}$. Then $\chi_{+}(f)=\chi_{+}(U v)=\phi_{\chi_{+}, v}^{U}$. For $p \in \mathbb{C}[T]^{S_{N}}$ we have

$$
\begin{aligned}
\left(L_{p}^{x} \phi_{\chi+, v}^{U}\right)(t, \gamma) & =\left(\operatorname{Res}\left(D_{p(Y)}^{x}\right)\left(\phi_{\chi+, v}^{U}\right)\right)(t, \gamma)=\phi_{\chi_{+}, v}^{\vartheta_{\mathcal{T}}\left(D_{p(Y)}^{x}\right) U}(t, \gamma) \\
& =\phi_{\chi+, v}^{\eta\left(p(Y)^{\dagger}\right) U}(t, \gamma)=p\left(\gamma^{-1}\right) \phi_{\chi_{+}, v}^{U}(t, \gamma)
\end{aligned}
$$

as meromorphic functions in $(t, \gamma) \in T \times T$, where the last equality follows from Lemma 6.10, (4.3) and the fact $p \in \mathbb{C}[T]^{S_{N}}$. Similarly,

$$
\begin{aligned}
\left(L_{p}^{y} \phi_{\chi+, v}^{U}\right)(t, \gamma) & =\left(\operatorname{Res}\left(D_{p(Y)}^{y}\right)\left(\phi_{\chi_{+}, v}^{U}\right)\right)(t, \gamma)=\phi_{\chi_{+}, v}^{\vartheta_{\mathcal{T}}\left(D_{p(Y)}^{y}\right) U}(t, \gamma) \\
& =\phi_{\chi_{+}, v}^{C_{\iota \iota}\left(\eta\left(p(Y)^{\ddagger}\right)\right) C_{\iota} U}(t, \gamma)=p(t) \phi_{\chi_{+}, v}^{U}(t, \gamma)
\end{aligned}
$$

as meromorphic functions in $(t, \gamma) \in T \times T$, hence $f=\phi_{\chi_{+}, v}^{U} \in \operatorname{BiSP}$.

\subsection{Bispectral Harish-Chandra series.}

Definition 6.17. We call $\Phi_{\kappa}^{+}:=\chi_{+}\left(\Phi_{\kappa}\right) \in$ BiSP the basic Harish-Chandra series solution of the bispectral problem.

Corollary 6.18. The solution $\Phi_{\kappa}^{+} \in \mathrm{BiSP}$ of the bispectral problem is selfdual, i.e.,

$$
\Phi_{\kappa}^{+}(t, \gamma)=\Phi_{\kappa}^{+}\left(\gamma^{-1}, t^{-1}\right)
$$

as meromorphic functions in $(t, \gamma) \in T \times T$.

Proof. By Theorem 5.6, we have

$$
\Phi_{\kappa}^{+}(t, \gamma)=\chi_{+}\left(C_{\iota} \Phi_{\kappa}\left(\gamma^{-1}, t^{-1}\right)\right)
$$

But $\chi_{+} C_{\iota}=\chi_{+}$, hence the result. 
Remark 6.19. In [17, for special values of $k$, the function $\Phi_{\kappa}^{+}$is constructed as formal power series in terms of generalized characters of Verma modules over the quantum group $\mathcal{U}_{q}\left(\mathfrak{s l}_{N}\right)$ (see also [15, [16]). The quantum group approach also leads to the self-duality of $\Phi_{\kappa}^{+}$; see [17, Thm. 5.6] (see [16]).

Note that $\Phi_{\kappa}^{+}=W_{\kappa} \Psi^{+}$with $\Psi^{+}=\chi_{+}(\Psi)$. For $\alpha \in Q_{+}$set

$$
\Gamma_{\alpha}^{+}(\gamma)=\chi_{+}\left(\Gamma_{\alpha}(\gamma)\right)
$$

as meromorphic function in $\gamma \in T$. By Lemma 5.7 and Theorem[5.10, $\Gamma_{\alpha}^{+}$is analytic at $T \backslash \mathcal{S}_{+}$and

$$
\Gamma_{0}^{+}(\gamma)=k^{\left(\begin{array}{c}
N \\
2
\end{array}\right)} K(\gamma)
$$

with $K$ given by (5.16). Recall that the solution space BiSP of the bispectral problem is $\mathbb{S}_{N^{-}}$-stable. In particular, we have solutions $\Phi_{w}^{+} \in \operatorname{BiSP}$ given by

$$
\Phi_{w}^{+}(t, \gamma):=\Phi_{\kappa}^{+}\left(t, w^{-1} \gamma\right)
$$

These are solutions of the bispectral problem which are asymptotically free in the asymptotic sector $\left\{t \in T|| t^{\alpha_{i}} \mid \gg 0 \forall 1 \leq i<N\right\}$ in the following sense: by Lemma 5.7 we have $\Phi_{w}^{+}(t, \gamma)=W_{\kappa}\left(t, w^{-1} \gamma\right) \Psi_{w}^{+}(t, \gamma)$ with $\Psi_{w}^{+}(t, \gamma):=\Psi^{+}\left(t, w^{-1} \gamma\right)$ admitting, for $\epsilon>0$ sufficiently small, the power series expansion

$$
\Psi_{w}^{+}(t, \gamma)=\sum_{\alpha \in Q_{+}} \Gamma_{\alpha}^{+}\left(w^{-1} \gamma\right) t^{-\alpha}
$$

for $(t, \gamma) \in B_{\epsilon}^{-1} \times T \backslash w\left(\mathcal{S}_{+}\right)$, converging normally in compacta of $B_{\epsilon}^{-1} \times T \backslash w\left(\mathcal{S}_{+}\right)$.

Proposition 6.20. The set of asymptotic solutions $\left\{\Phi_{w}^{+}\right\}_{w \in S_{N}} \subset$ BiSP of the bispectral problem is $\mathbb{F}$-linearly independent.

Proof. Suppose that

$$
\sum_{w \in S_{N}} a_{w}(t, \gamma) \Phi_{w}^{+}(t, \gamma)=0
$$

as meromorphic functions in $(t, \gamma) \in T \times T$ with coefficients $a_{w} \in \mathbb{F}\left(w \in S_{N}\right)$. Replacing $t$ by $q^{-m \delta} t(m \in \mathbb{N})$ and using (5.5) we obtain

$$
\sum_{w \in S_{N}} k^{-m\langle\delta, \delta\rangle} \gamma^{-m w w_{0}(\delta)} a_{w}(t, \gamma) W_{\kappa}\left(t, w^{-1} \gamma\right) \Psi_{w}^{+}\left(q^{-m \delta} t, \gamma\right)=0
$$

as meromorphic functions in $(t, \gamma) \in T \times T$. Fix $u \in S_{N}$. We are going to derive from (6.16) that $a_{u}=0$. For this we will use the fact that for $w \neq u$,

$$
\lim _{m \rightarrow \infty} \zeta^{m\left(u w_{0}(\delta)-w w_{0}(\delta)\right)}=\lim _{m \rightarrow \infty}\left(w_{0} u^{-1} \zeta\right)^{m\left(\delta-w_{0} u^{-1} w w_{0}(\delta)\right)}=0
$$

if $\zeta \in u w_{0}\left(B_{1}\right)$.

Recall the $W$-invariant subset $\mathcal{S} \subset T$ (see (4.6)), which contains $\mathcal{S}_{+}$. For generic $\zeta \in T$ (concretely, $\zeta \notin \mathcal{S}$, and $a_{w}(t, \gamma)$ and $W_{\kappa}\left(t, w^{-1} \gamma\right)$ specializable at $\gamma=\zeta$ for all $w \in S_{N}$ ), it follows from Proposition 5.9 and (6.16) that, for all $m \in \mathbb{N}$,

$$
\sum_{w \in S_{N}} \zeta^{m\left(u w_{0}(\delta)-w w_{0}(\delta)\right)} a_{w}(t, \zeta) W_{\kappa}\left(t, w^{-1} \zeta\right) \Psi_{w}^{+}\left(q^{-m \delta} t, \zeta\right)=0
$$

as meromorphic function in $t \in T$. Using (6.17) and the power series expansion (6.15), the limit $m \rightarrow \infty$ of (6.18) yields, for generic $\zeta \in u w_{0}\left(B_{1}\right)$,

$$
a_{u}(t, \zeta) W_{\kappa}\left(t, u^{-1} \zeta\right) \Gamma_{0}^{+}\left(u^{-1} \zeta\right)=0
$$

as meromorphic function in $t \in T$. This implies $a_{u}=0$, as desired. 
Corollary 6.21. The map $\chi_{+}: \mathrm{SOL} \rightarrow \mathrm{BiSP}$ is injective.

Proof. Note that $\chi_{+}\left(\tau(e, w) \Phi_{\kappa}\right)=\Phi_{w}^{+}\left(w \in S_{N}\right)$. The statement follows now directly from Proposition 6.20 and Proposition 5.13.

6.4. Specialized central character and Harish-Chandra series. We write

$$
\mathrm{SP}_{\zeta}=\left\{f \in \mathcal{M}(T) \mid L_{p}^{x} f=p\left(\zeta^{-1}\right) f \quad \forall p \in \mathbb{C}[T]^{S_{N}}\right\}
$$

for the spectral problem of the Ruijsenaars $q$-difference operators with fixed spectral parameter $\zeta \in T$. Note that $\mathrm{SP}_{\zeta} \subset H_{0}^{\mathcal{M}(T)}$ is $S_{N}$-stable, with $S_{N}$-action on $H_{0}^{\mathcal{M}(T)}$ given by $(w f)(t)=f\left(w^{-1} t\right)$ for $f \in H_{0}^{\mathcal{M}(T)}$ and $w \in S_{N}$.

By [13, Prop. 5.2], the quantum KZ equations (4.10) are consistent for all values $\zeta \in T$ of the central character. The arguments from Subsection 6.2, applied to the quantum $\mathrm{KZ}$ equations (4.10) for fixed $\zeta$ and with the role of $U$ taken over by an invertible matrix solution $U_{\zeta}$ of (4.10), result in the following special case of the Cherednik-Matsuo correspondence from [6, 7] (concretely, in the notations of [7, take the principal series module $V=M(\zeta)$ in [7, Thm. 4.2] and let $\tau$ be the projection from $M(\zeta)$, along the direct sum decomposition of $M(\zeta)$ in $H_{0}$-isotypical components, onto the trivial component).

Proposition 6.22. Let $\zeta \in T$. Then $\chi_{+}$defines an $\mathcal{E}(T)$-linear $S_{N}$-equivariant map $\chi_{+}: \mathrm{SOL}_{\zeta} \rightarrow \mathrm{SP}_{\zeta}$.

For a further analysis of the map $\chi_{+}: \mathrm{SOL}_{\zeta} \rightarrow \mathrm{SP}_{\zeta}$, we refer to [7] and [9, $\S 1.3 .4]$.

Harish-Chandra type series solutions of the spectral problem of the Ruijsenaars $q$-difference operators $L_{p}^{x}\left(p \in \mathbb{C}[T]^{S_{N}}\right)$ with fixed spectral parameter $\zeta \in T$ were studied in, e.g., [15] and [27] (see also [31] for arbitrary root systems). The results of the previous subsection allow us to reobtain these solutions by specialization of the basic Harish-Chandra series $\Phi_{\kappa}^{+}$. It leads to new results on the convergence and singularities of these solutions, which we state now explicitly.

By Subsection 5.4, for generic $\kappa \in \mathbb{C}^{\times}$the basic Harish-Chandra series $\Phi_{\kappa}^{+}(t, \gamma)$ is specializable at $\gamma=\zeta$ when $\zeta \in T \backslash \mathcal{S}_{+}$. Concretely, for $\zeta \in T \backslash \mathcal{S}_{+}$and generic $\kappa \in \mathbb{C}^{\times}$, we can write

$$
\Phi_{\kappa}^{+}(t, \zeta)=W_{\kappa}(t, \zeta) \Psi^{+}(t, \zeta)
$$

as meromorphic function in $t \in T$, where $\Psi^{+}=\chi_{+}(\Psi)$ (see Subsection 6.3). Due to the results in Subsection 5.4 (see Proposition 5.8) we obtain the following result.

Corollary 6.23. For $\zeta \in T \backslash \mathcal{S}_{+}$, the meromorphic function $\Psi^{+}(t, \zeta)$ in $t \in T$ is analytic at $t \in T \backslash \mathcal{S}_{+}^{-1}$.

Let $\zeta \in T \backslash \mathcal{S}$, where $\mathcal{S} \subset T$ is the $W$-invariant set (4.6). For $\kappa \in \mathbb{C}^{\times}$such that $W_{\kappa}\left(t, w^{-1} \gamma\right)$ may be specialized at $\gamma=\zeta$ for all $w \in S_{N}$, the asymptotic solutions $\Phi_{w}^{+}(t, \gamma)\left(w \in S_{N}\right)$ of the bispectral problem (see (6.14) $)$ may thus be specialized at $\gamma=\zeta$, giving rise to solutions $\Phi_{w}^{+}(\cdot ; \zeta) \in \mathrm{SP}_{\zeta}\left(w \in S_{N}\right)$; see Corollary 5.14 and Proposition 6.22, Observe that for $\epsilon>0$ sufficiently small,

$$
\Phi_{w}^{+}(t, \zeta)=W_{\kappa}\left(t, w^{-1} \zeta\right) \sum_{\alpha \in Q_{+}} \Gamma_{\alpha}^{+}\left(w^{-1} \zeta\right) t^{-\alpha}
$$

for $t \in B_{\epsilon}^{-1}$, with normal convergence of the power series on compacta of $B_{\epsilon}^{-1}$. Since $\zeta \notin \mathcal{S}$ we furthermore have

$$
\Gamma_{0}^{+}\left(w^{-1} \zeta\right)=k^{\left(\begin{array}{c}
N \\
2
\end{array}\right)} K\left(w^{-1} \zeta\right) \neq 0,
$$


with $K$ given by (5.16).

Definition 6.24. Let $\zeta \in T \backslash \mathcal{S}$. The $\Phi_{w}^{+}(\cdot ; \zeta) \in \mathrm{SP}_{\zeta}\left(w \in S_{N}\right)$ are the HarishChandra series solutions of the spectral problem $L_{p}^{x} f=p\left(\zeta^{-1}\right) f\left(p \in \mathbb{C}[T]^{S_{N}}\right)$.

Remark 6.25. In [15] (and [31) the Harish-Chandra series are investigated as formal power series solutions to the spectral problem of the Ruijsenaars operators. The advantage of the present approach is the fact that it implies the convergence of the formal power series, basically as a consequence of a general statement about convergence of formal power series solutions of holonomic systems of $q$-difference equations (see the appendix). Chalykh's [5] Baker-Akhiezer functions arise as Harish-Chandra series solutions for special values of $k$; see [31, §4.4]. In [27, the Harish-Chandra series solutions of the Ruijsenaars operators are constructed as matrix coefficients of products of vertex operators. By this approach, one obtains an explicit integral representation of the Harish-Chandra series.

Remark 6.26. Observe that

$$
\lim _{\substack{\lambda \in \Lambda \\
\lambda \rightarrow \infty}} \frac{\Phi_{\kappa}^{+}\left(t, q^{\lambda} k^{-\delta}\right)}{W_{\kappa}\left(t, q^{\lambda} k^{-\delta}\right)}=\Gamma_{0}^{+}\left(t^{-1}\right)=k^{\left(\begin{array}{c}
N \\
2
\end{array}\right)} K\left(t^{-1}\right),
$$

with $\lambda \rightarrow \infty$ meaning $\lambda_{i}-\lambda_{i+1} \rightarrow \infty$ for all $1 \leq i<N$. Thus, $K$ (see (5.16)) is a normalized limit of the asymptotic solutions $\Phi_{\kappa}^{+}\left(\cdot, q^{\lambda} k^{-\delta}\right) \in \mathrm{SP}_{q^{\lambda} k^{-\delta}}$. The solution space $\mathrm{SP}_{q^{\lambda} k^{-\delta}}$ contains the symmetric Macdonald polynomial of degree $\lambda \in \Lambda$. It turns out though that $\Phi_{\kappa}^{+}\left(\cdot, q^{\lambda} k^{-\delta}\right)$ is not a multiple of the Macdonald polynomial of degree $\lambda \in \Lambda$, but $\Phi_{\kappa}^{+}\left(\cdot, q^{w_{0}(\lambda)} k^{\delta}\right.$ ) is (this will become apparent in the next section). On the other hand, the leading coefficient $K$ (see (5.16)) also naturally appears as a normalized limit of the Macdonald polynomial when the degree $\lambda \in \Lambda$ of the polynomial tends to infinity; see [10, Lemma 4.3] (this limit was proven in [40] in the $L^{2}$-sense).

\section{Polynomial theory}

We assume throughout this section that $k \in \mathbb{C}^{\times}$satisfies the generic conditions

$$
\begin{array}{rlrl}
k^{2 j} & \notin q^{\mathbb{Z}}, & & \forall 1 \leq j \leq N, \\
k^{\left\langle\delta, \varpi_{j}-w\left(\varpi_{j}\right)\right\rangle} \notin q^{\mathbb{Z}}, & & \forall 1 \leq j<N, \forall w \in S_{N}: w\left(\varpi_{j}\right) \neq \varpi_{j} .
\end{array}
$$

7.1. Polynomial solutions of the quantum $\mathrm{KZ}$ equation. We are going to use a special case of Proposition 4.9 to create $S_{N^{-}}$-invariant (with respect to the $S_{N^{-}}$ action $\varsigma$ on $\mathrm{SOL}_{\zeta}$; see (4.11) ) polynomial solutions of the quantum $\mathrm{KZ}$ equations.

Lemma 7.1. Let $\lambda \in \Lambda$. The possible poles of the $\mathbb{C}[T] \otimes \operatorname{End}\left(H_{0}\right)$-valued rational function

$$
\gamma \mapsto C_{(e,-\lambda)}\left(\cdot, q^{\lambda} \gamma\right)=C_{(e, \lambda)}(\cdot, \gamma)^{-1}
$$

in $\gamma \in T$ are at $\gamma^{\alpha} \in k^{2} q^{-\mathbb{N}}$ for some $\alpha \in R^{+}$. The possible poles of

$$
\gamma \mapsto C_{(e, \lambda)}(\cdot, \gamma)
$$

are at $\gamma^{\alpha} \in k^{-2} q^{-\mathbb{N}}$ for some $\alpha \in R^{+}$.

Proof. Since $R_{i}(z)$ has only a (simple) pole at $z=k^{-2}$, this follows from (5.13) and the cocycle property of $C$; see Lemma 5.7 
Since $k$ satisfies $k^{2 j} \notin q^{\mathbb{Z}}$ for $1 \leq j \leq N$ by (7.1), the spectrum of $\eta_{q^{\lambda} k^{-\delta}}\left(\mathbb{C}_{Y}[T]\right)$ is simple and the $\xi_{w}\left(q^{\lambda} k^{-\delta}\right)\left(w \in S_{N}\right)$ form a $\mathbb{C}$-basis of $H_{0}$ for all $\lambda \in \Lambda$. Furthermore, for such $k$ we have that $\gamma \mapsto C_{(e, \lambda)}(\cdot, \gamma)^{ \pm 1}$ is regular at $\gamma=k^{-\delta}$ for all $\lambda \in \Lambda$; see Lemma 7.1. The additional conditions on $k$ in (7.1) will play a role in Subsection 7.3 and Subsection 7.4

Proposition 4.9 now immediately implies the following result.

Corollary 7.2. Let $\lambda \in \Lambda$. Then $f \mapsto C_{(e, \lambda)}\left(\cdot, k^{-\delta}\right)^{-1} f$ defines an $S_{N}$-equivariant isomorphism $\mathrm{SOL}_{k^{-\delta}} \rightarrow \mathrm{SOL}_{q^{\lambda} k^{-\delta}}$.

The special interest in the quantum KZ equations for the particular central characters $\gamma=q^{\lambda} k^{-\delta}(\lambda \in \Lambda)$ comes from the fact that it admits $S_{N}$-invariant polynomial solutions. The key step in deriving this result is the following lemma.

Lemma 7.3. The element $v_{+}:=\sum_{w \in S_{N}} k^{\ell(w)} T_{w} \in H_{0}$ is a constant $S_{N}$-invariant solution of the quantum $K Z$ equation with central character $k^{-\delta}$. In other words,

$$
C_{\lambda}^{k^{-\delta}}(t) v_{+}=v_{+}, \quad \forall \lambda \in \mathbb{Z}^{N} .
$$

Proof. Note that $R_{i}(z) v_{+}=v_{+}$, so by Lemma4.1 and Lemma4.5(ii), for any $\zeta \in T$,

$$
C_{\varpi_{i}}^{\zeta}(t) v_{+}=\eta_{\zeta}(\pi)^{i} v_{+}=\sum_{w \in S_{N}} k^{\ell(w)} \zeta^{w^{-1} w_{0} \varpi_{i}} T_{\sigma^{i} w}=\sum_{w \in S_{N}} k^{\ell\left(\sigma^{-i} w\right)} \zeta^{w^{-1} \varpi_{i}} T_{w} .
$$

Then use $\ell\left(\sigma^{-i} w\right)-\ell(w)=\left\langle\delta, w^{-1} \varpi_{i}\right\rangle$ for $1 \leq i \leq N$ (for the proof of this formula, it suffices to prove it for $i=1$. In that case, look at the positive roots that are mapped to negative roots by $\left.\sigma^{-1} w\right)$. It implies that

$$
C_{\varpi_{i}}^{\zeta}(t) v_{+}=\sum_{w \in S_{N}} k^{\ell(w)}\left(k^{\delta} \zeta\right)^{w^{-1} \varpi_{i}} T_{w} .
$$

In particular, $C_{\varpi_{i}}^{k^{-\delta}}(t) v_{+}=v_{+}$for all $i$. Note, furthermore, that $R_{i}(z) v_{+}=v_{+}$ implies that $\varsigma\left(s_{i}\right) v_{+}=C_{s_{i}}^{k^{-\delta}}(t) v_{+}=v_{+}$for all $1 \leq i<N$ (with $\varsigma$ given by (4.11)). Hence, $v_{+} \in \mathrm{SOL}_{k^{-\delta}}$ is $S_{N^{-i n v a r i a n t} \text {. }}$

Proposition 7.4. For $\lambda \in \Lambda$, the nonzero $S_{N}$-invariant solution

$$
Q_{\lambda}:=C_{(e, \lambda)}\left(\cdot, k^{-\delta}\right)^{-1} v_{+} \in \mathrm{SOL}_{q^{\lambda} k^{-\delta}}
$$

of the quantum $K Z$ equation is an $H_{0}$-valued Laurent polynomial on $T$ satisfying

$$
Q_{\lambda}(t)=\sum_{\alpha \in Q_{+}} K_{\alpha}(\lambda) t^{\lambda-\alpha},
$$

with $K_{\alpha}(\lambda) \in H_{0}$ (all but finitely many terms zero).

Proof. Note that Corollary 7.2 and Lemma 7.3 imply that $0 \neq Q_{\lambda} \in \mathrm{SOL}_{q^{\lambda} k^{-\delta}}$ and that $Q_{\lambda}$ is $S_{N}$-invariant. The triangularity property (7.2) follows from the cocycle property, (5.13), the explicit form of the $R_{i}(z)$ and the fact that

$$
\eta(\pi)\left(t^{-1}\right)^{-i} T_{w}=t^{w^{-1} \varpi_{i}} T_{\sigma^{-i} w}, \quad w \in S_{N},
$$

which is a direct consequence of Lemma 4.1 


\subsection{Duality.}

Lemma 7.5. For $\lambda \in \Lambda$, we have $Q_{\lambda}\left(k^{\delta}\right)=v_{+}$.

Proof. Since $v_{+} \in \mathrm{SOL}_{k^{-\delta}}$ and $C_{\iota} v_{+}=v_{+}$, we obtain

$$
\begin{aligned}
Q_{\lambda}\left(k^{\delta}\right) & =C_{(e,-\lambda)}\left(k^{\delta}, q^{\lambda} k^{-\delta}\right) v_{+} \\
& =C_{\iota} C_{(-\lambda, e)}\left(q^{-\lambda} k^{\delta}, k^{-\delta}\right) C_{\iota} v_{+}=v_{+},
\end{aligned}
$$

for $\lambda \in \Lambda$.

The polynomial solutions $Q_{\lambda}$ of the quantum $\mathrm{KZ}$ equations are self-dual in the following sense.

Proposition 7.6. For $\lambda, \mu \in \Lambda$, we have

$$
Q_{\lambda}\left(q^{-\mu} k^{\delta}\right)=C_{\iota} Q_{\mu}\left(q^{-\lambda} k^{\delta}\right) .
$$

Proof. For $\lambda, \mu \in \Lambda$ we have, using $v_{+} \in \mathrm{SOL}_{k^{-\delta}}$ and the previous lemma,

$$
\begin{aligned}
Q_{\lambda}\left(q^{-\mu} k^{\delta}\right) & =C_{(e,-\lambda)}\left(q^{-\mu} k^{\delta}, q^{\lambda} k^{-\delta}\right) v_{+} \\
& =C_{(e,-\lambda)}\left(q^{-\mu} k^{\delta}, q^{\lambda} k^{-\delta}\right) C_{(-\mu, e)}\left(q^{-\mu} k^{\delta}, k^{-\delta}\right) v_{+} \\
& =C_{(-\mu,-\lambda)}\left(q^{-\mu} k^{\delta}, q^{\lambda} k^{-\delta}\right) v_{+} .
\end{aligned}
$$

Since $C_{(-\mu,-\lambda)}\left(q^{-\mu} k^{\delta}, q^{\lambda} k^{-\delta}\right)=C_{\iota} C_{(-\lambda,-\mu)}\left(q^{-\lambda} k^{\delta}, q^{\mu} k^{-\delta}\right) C_{\iota}$ and $C_{\iota} v_{+}=v_{+}$, we conclude from (7.3) that $Q_{\lambda}\left(q^{-\mu} k^{\delta}\right)=C_{\iota} Q_{\mu}\left(q^{-\lambda} k^{\delta}\right)$.

7.3. Relation to the basic asymptotically free solution. In this subsection, we relate the polynomial solutions $Q_{\lambda}(\lambda \in \Lambda)$ of the quantum $\mathrm{KZ}$ equations to the basic asymptotic solution $\Phi_{\kappa}$. Some care is needed though: it is not possible to specialize all the asymptotic solutions $C_{(e, w)}(t, \gamma) \Phi_{\kappa}\left(t, w^{-1} \gamma\right)\left(w \in S_{N}\right)$ to $\gamma=q^{\lambda} k^{-\delta}(\lambda \in \Lambda)$ since $q^{\lambda} k^{-\delta} \in \mathcal{S}$; see Corollary [5.14] We shall see that $C_{\left(e, w_{0}\right)}(t, \gamma) \Phi_{\kappa}\left(t, w_{0} \gamma\right)$ can be specialized at $\gamma=q^{\lambda} k^{-\delta}$, which is sufficient for our purposes.

Lemma 7.7. Let $\lambda \in \Lambda$. There exists a unique $\Xi_{\lambda} \in \mathrm{SOL}_{q^{\lambda} k^{-\delta}}$ such that, for $\epsilon>0$ sufficiently small, we have an $H_{0}$-valued power series expansion

$$
\Xi_{\lambda}(t)=\sum_{\alpha \in Q^{+}} \widetilde{\Gamma}_{\alpha}(\lambda) t^{\lambda-\alpha}
$$

converging normally on compacta of $B_{\epsilon}^{-1}$ and with leading coefficient

$$
\widetilde{\Gamma}_{0}(\lambda)=\eta_{q^{\lambda} k^{-\delta}}\left(T_{w_{0}}\right) \xi_{w_{0}}\left(q^{\lambda} k^{-\delta}\right) .
$$

Proof. Consider the gauged quantum KZ equations for $1 \leq i \leq N$,

$$
\widetilde{A}_{i}(t) \widetilde{\Xi}\left(q^{-\varpi_{i}} t\right)=\widetilde{\Xi}(t), \quad \widetilde{\Xi} \in H_{0}^{\mathcal{M}(T)},
$$

with $q$-connection matrices $\widetilde{A}_{i}(t)=q^{-\left\langle\lambda, \varpi_{i}\right\rangle} C_{\left(\varpi_{i}, e\right)}\left(t, q^{\lambda} k^{-\delta}\right)$. Note that $\widetilde{A}_{N}(t)=$ id; see Lemma 5.2. Observe that $\widetilde{\Xi}$ is a solution of the holonomic system (7.4) of $q$-difference equations if and only if $x^{\lambda} \widetilde{\Xi} \in \mathrm{SOL}_{q^{\lambda} k^{-\delta}}$. By Corollary 4.6, we have $\widetilde{A}_{i} \in Q_{0}(\mathcal{A}) \otimes \operatorname{End}\left(H_{0}\right)$ and

$$
\widetilde{A}_{i}^{(0)}=q^{-\left\langle\lambda, \varpi_{i}\right\rangle} k^{\left\langle\delta, \varpi_{i}\right\rangle} \eta_{q^{\lambda} k^{-\delta}}\left(T_{w_{0}} Y^{w_{0}\left(\varpi_{i}\right)} T_{w_{0}}^{-1}\right) .
$$


The $\widetilde{A}_{i}^{(0)}(1 \leq i<N)$ are semisimple endomorphisms of $H_{0}$. A basis of simultaneous eigenvectors of $H_{0}$ is given by $\eta_{q^{\lambda} k^{-\delta}}\left(T_{w_{0}}\right) \xi_{w}\left(q^{\lambda} k^{-\delta}\right)\left(w \in S_{N}\right)$. In fact,

$$
\widetilde{A}_{i}^{(0)}\left(\eta_{q^{\lambda} k^{-\delta}}\left(T_{w_{0}}\right) \xi_{w}\left(q^{\lambda} k^{-\delta}\right)\right)=\gamma_{w, i} \eta_{q^{\lambda} k^{-\delta}}\left(T_{w_{0}}\right) \xi_{w}\left(q^{\lambda} k^{-\delta}\right)
$$

for all $1 \leq i<N$ and $w \in S_{N}$ with

$$
\gamma_{w, i}=q^{\left\langle\lambda, w^{-1} w_{0}\left(\varpi_{i}\right)-\varpi_{i}\right\rangle} k^{\left\langle\delta, \varpi_{i}-w^{-1} w_{0}\left(\varpi_{i}\right)\right\rangle} ;
$$

see (5.10). Note, in particular, that $\gamma_{w, i} \notin q^{-\mathbb{N}}$ for all $w \in S_{N}$ and all $1 \leq i<N$ by the generic conditions (7.1) on $k$, and that $\gamma_{w_{0}, i}=1$ for all $1 \leq i<N$. Hence, Theorem 8.6 in the appendix, applied to (7.4) by taking $M=N-1, q_{i}=q$ and variables $z_{i}=x^{-\alpha_{i}}(1 \leq i<N)$ shows that there exists a unique $\widetilde{\Xi} \in \mathcal{M}(T) \otimes H_{0}$ satisfying (7.4) and admitting an $H_{0}$-valued power series expansion

$$
\widetilde{\Xi}(t)=\sum_{\alpha \in Q^{+}} \widetilde{\Gamma}_{\alpha}(\lambda) t^{-\alpha}
$$

converging normally on compacta of $B_{\epsilon}^{-1}$ for some $\epsilon>0$ small enough, and having as leading coefficient $\widetilde{\Gamma}_{0}(\lambda)=\eta_{q^{\lambda} k^{-\delta}}\left(T_{w_{0}}\right) \xi_{w_{0}}\left(q^{\lambda} k^{-\delta}\right)$. This directly implies the lemma.

Recall that the cocycle values $C_{(e, w)}(t, \gamma)\left(w \in S_{N}\right)$ are independent of $t \in T$. We suppress $t$ from the notation and simply write $C_{(e, w)}(\gamma)$. Recall that $C_{(e, w)}(\gamma)$ for $w \in S_{N}$ is an $\operatorname{End}\left(H_{0}\right)$-valued regular function in $\gamma \in T$.

Theorem 7.8. Fix $\lambda \in \Lambda$. For $\kappa \notin q^{\mathbb{Z}}$, the basic asymptotic solution $\Phi_{\kappa}(t, \gamma)$ of $B q K Z$ can be specialized at $\gamma=q^{w_{0}(\lambda)} k^{\delta}$, giving rise to a $H_{0}$-valued meromorphic function $\Phi_{\kappa}\left(t, q^{w_{0}(\lambda)} k^{\delta}\right)$ in $t \in T$. Then

$$
Q_{\lambda}(t)=r_{\kappa} C_{\left(e, w_{0}\right)}\left(q^{\lambda} k^{-\delta}\right) \Phi_{\kappa}\left(t, q^{w_{0}(\lambda)} k^{\delta}\right)
$$

with

$$
r_{\kappa}=\theta(\kappa)^{N} k^{-\left(\begin{array}{c}
N \\
2
\end{array}\right)} \prod_{1 \leq i<j \leq N} \frac{\left(k^{2(j-i+1)} ; q\right)_{\infty}}{\left(k^{2(j-i)} ; q\right)_{\infty}} .
$$

Proof. We first show that both $Q_{\lambda}$ and the right-hand side of (7.6) are nonzero scalar multiples of $\Xi_{\lambda}$.

We start with the right-hand side of (7.6). Since $\Phi$ is $\mathbb{S}_{N}$-stable, we have

$$
\Phi_{w_{0}}:=\tau\left(e, w_{0}\right) \Phi_{\kappa} \in \mathrm{SOL} .
$$

Concretely, it is given by

$$
\Phi_{w_{0}}(t, \gamma)=C_{\left(e, w_{0}\right)}(\gamma) \Phi_{\kappa}\left(t, w_{0}(\gamma)\right)=W_{\kappa}\left(t, w_{0}(\gamma)\right) C_{\left(e, w_{0}\right)}(\gamma) \Psi\left(t, w_{0}(\gamma)\right) .
$$

Since $w_{0}\left(q^{\lambda} k^{-\delta}\right)=q^{w_{0}(\lambda)} k^{\delta} \notin \mathcal{S}_{+}$by (7.1), we may, in view of Proposition 5.9, specialize $\Phi_{w_{0}}(t, \gamma)$ at $\gamma=q^{\lambda} k^{-\delta}$, obtaining $\Phi_{w_{0}}\left(\cdot, q^{\lambda} k^{-\delta}\right) \in \mathrm{SOL}_{q^{\lambda}} k^{-\delta}$. By (5.3) we have

$$
W_{\kappa}\left(t, w_{0}\left(q^{\lambda} k^{-\delta}\right)\right)=k^{\langle\delta, \lambda\rangle} \theta(\kappa)^{-N} t^{\lambda},
$$

hence by Proposition 5.9

$$
\Phi_{w_{0}}\left(t, q^{\lambda} k^{-\delta}\right)=k^{\langle\delta, \lambda\rangle} \theta(\kappa)^{-N} \sum_{\alpha \in Q_{+}} \Gamma_{\alpha}^{w_{0}} t^{\lambda-\alpha}
$$


with $\Gamma_{\alpha}^{w_{0}}=C_{\left(e, w_{0}\right)}\left(q^{\lambda} k^{-\delta}\right) \Gamma_{\alpha}\left(q^{w_{0}(\lambda)} k^{\delta}\right)$. From the definitions of $C_{\left(e, w_{0}\right)}, d_{w}, \eta$ and $\xi_{w_{0}}$ (see Subsections 2.3, 3.1 and 4.1) we have

$$
\begin{aligned}
C_{\left(e, w_{0}\right)}(\gamma) T_{w_{0}} & =d_{w_{0}}\left(\gamma^{-1}\right)^{-1} \eta\left(T_{w_{0}}\right) \xi_{w_{0}}(\gamma) \\
& =\left(\prod_{\alpha \in R^{+}} \frac{1}{k-k^{-1} \gamma^{\alpha}}\right) \eta\left(T_{w_{0}}\right) \xi_{w_{0}}(\gamma)
\end{aligned}
$$

as $H_{0}$-valued regular functions in $\gamma \in T$. By Theorem [5.10, the leading coefficient $\Gamma_{0}^{w_{0}}$ thus simplifies to

$$
\begin{aligned}
\Gamma_{0}^{w_{0}} & =K\left(q^{w_{0}(\lambda)} k^{\delta}\right) C_{\left(e, w_{0}\right)}\left(q^{\lambda} k^{-\delta}\right) T_{w_{0}} \\
& =K\left(q^{w_{0}(\lambda)} k^{\delta}\right) d_{w_{0}}\left(q^{-\lambda} k^{\delta}\right)^{-1} \eta_{q^{\lambda} k^{-\delta}}\left(T_{w_{0}}\right) \xi_{w_{0}}\left(q^{\lambda} k^{-\delta}\right),
\end{aligned}
$$

where $K$ is given by (5.16). Combined with the previous lemma, we conclude that

$$
\Phi_{w_{0}}\left(t, q^{\lambda} k^{-\delta}\right)=k^{\langle\delta, \lambda\rangle} \theta(\kappa)^{-N} K\left(q^{w_{0}(\lambda)} k^{\delta}\right) d_{w_{0}}\left(q^{-\lambda} k^{\delta}\right)^{-1} \Xi_{\lambda}(t)
$$

In view of (7.1), $\Phi_{w_{0}}\left(t, q^{\lambda} k^{-\delta}\right)$ thus is a nonzero constant multiple of $\Xi_{\lambda}(t)$.

Next, we consider $0 \neq Q_{\lambda} \in \mathrm{SOL}_{q^{\lambda} k^{-\delta}}$. By Lemma 7.7 and (17.2), it suffices to note that $K_{0}(\lambda)$ is a constant multiple of $\eta_{q^{\lambda} k^{-\delta}}\left(T_{w_{0}}\right) \xi_{w_{0}}\left(q^{\lambda} k^{-\delta}\right)$, which follows directly from the fact that $K_{0}(\lambda) \in H_{0}$ satisfies

$$
\widetilde{A}_{i}^{(0)} K_{0}(\lambda)=K_{0}(\lambda), \quad \forall 1 \leq i \leq N
$$

where $\widetilde{A}_{i}$ is given by (7.5); see the proof Lemma of 7.7. Thus, $Q_{\lambda}(t)$ is a nonzero constant multiple of $\Xi_{\lambda}(t)$, and we conclude that

$$
Q_{\lambda}(t)=r_{\kappa}(\lambda) \Phi_{w_{0}}\left(t, q^{\lambda} k^{-\delta}\right),
$$

for some $r_{\kappa}(\lambda) \in \mathbb{C}^{\times}$. We first show that $r_{\kappa}(\lambda)$ is independent of $\lambda \in \Lambda$.

For $w \in S_{N}$, we write $C_{(w, e)}(t)$ for the $\gamma$-independent value $C_{(w, e)}(t, \gamma)$ of the cocycle. Let $\lambda, \mu \in \Lambda$. By the $S_{N}$-invariance of $Q_{\lambda}$, we then have, on the one hand,

$$
\begin{aligned}
Q_{\lambda}\left(q^{-\mu} k^{\delta}\right) & =C_{\left(w_{0}, e\right)}\left(q^{-\mu} k^{\delta}\right) Q_{\lambda}\left(q^{-w_{0}(\mu)} k^{-\delta}\right) \\
& =r_{\kappa}(\lambda) C_{\left(w_{0}, e\right)}\left(q^{-\mu} k^{\delta}\right) C_{\left(e, w_{0}\right)}\left(q^{\lambda} k^{-\delta}\right) \Phi_{\kappa}\left(q^{-w_{0}(\mu)} k^{-\delta}, q^{w_{0}(\lambda)} k^{\delta}\right) \\
& =r_{\kappa}(\lambda) C_{\left(w_{0}, w_{0}\right)}\left(q^{-\mu} k^{\delta}, q^{\lambda} k^{-\delta}\right) \Phi_{\kappa}\left(q^{-w_{0}(\mu)} k^{-\delta}, q^{w_{0}(\lambda)} k^{\delta}\right) .
\end{aligned}
$$

On the other hand, using the self-duality of $Q_{\lambda}$ (see Proposition 7.6) and of $\Phi_{\kappa}$ (see Theorem [5.6),

$$
\begin{aligned}
Q_{\lambda}\left(q^{-\mu} k^{\delta}\right) & =C_{\iota} Q_{\mu}\left(q^{-\lambda} k^{\delta}\right)=C_{\iota} C_{\left(w_{0}, e\right)}\left(q^{-\lambda} k^{\delta}\right) Q_{\mu}\left(q^{-w_{0}(\lambda)} k^{-\delta}\right) \\
& =r_{\kappa}(\mu) C_{\iota} C_{\left(w_{0}, e\right)}\left(q^{-\lambda} k^{\delta}\right) C_{\left(e, w_{0}\right)}\left(q^{\mu} k^{-\delta}\right) \Phi_{\kappa}\left(q^{-w_{0}(\lambda)} k^{-\delta}, q^{w_{0}(\mu)} k^{\delta}\right) \\
& =r_{\kappa}(\mu) C_{\iota} C_{\left(w_{0}, w_{0}\right)}\left(q^{-\lambda} k^{\delta}, q^{\mu} k^{-\delta}\right) \Phi_{\kappa}\left(q^{-w_{0}(\lambda)} k^{-\delta}, q^{w_{0}(\mu)} k^{\delta}\right) \\
& =r_{\kappa}(\mu) C_{\left(w_{0}, w_{0}\right)}\left(q^{-\mu} k^{\delta}, q^{\lambda} k^{-\delta}\right) C_{\iota} \Phi_{\kappa}\left(q^{-w_{0}(\lambda)} k^{-\delta}, q^{w_{0}(\mu)} k^{\delta}\right) \\
& =r_{\kappa}(\mu) C_{\left(w_{0}, w_{0}\right)}\left(q^{-\mu} k^{\delta}, q^{\lambda} k^{-\delta}\right) \Phi_{\kappa}\left(q^{-w_{0}(\mu)} k^{-\delta}, q^{w_{0}(\lambda)} k^{\delta}\right) .
\end{aligned}
$$

We conclude that $r_{\kappa}(\lambda)=r_{\kappa}(\mu)$ if $Q_{\lambda}\left(q^{-\mu} k^{\delta}\right) \neq 0$. In particular, since $Q_{\lambda}\left(k^{\delta}\right)=$ $v_{+} \neq 0$, we have $r_{\kappa}(\lambda)=r_{\kappa}(0)$ for all $\lambda \in \Lambda$.

It remains to compute $r_{\kappa}:=r_{\kappa}(0)$. Using the fact that $C_{\left(e, s_{i}\right)}(\gamma)=C_{\iota} R_{i}\left(\gamma^{-\alpha_{i}}\right) C_{\iota}$, with $R_{i}(z)=c_{k}(z)^{-1}\left(\eta\left(T_{i}^{-1}\right)-k^{-1}\right)+1$ for $1 \leq i<N$, as well as that $C_{\iota}\left(T_{w^{-1}}^{-1} T_{w_{0}}\right)=$ 
$T_{w_{0} w^{-1}}$ for all $w \in S_{N}$, we get $C_{\left(e, w_{0}\right)}(\gamma) T_{w_{0}}=\sum_{w \leq w_{0}} e_{w}(\gamma) T_{w_{0} w^{-1}}$ as $H_{0^{-} \text {-valued }}$ regular function in $\gamma \in T$ with $e_{w} \in \mathbb{C}[T]$ and with

$$
e_{w_{0}}(\gamma)=\prod_{\beta \in R^{+}} c_{k}\left(\gamma^{-\beta}\right)^{-1} .
$$

Taking the $T_{e}$-coefficient in the expansion of the formula

$$
v_{+}=Q_{0}=r_{\kappa} \Phi_{w_{0}}\left(\cdot, k^{-\delta}\right)=r_{\kappa} \theta(\kappa)^{-N} K\left(k^{\delta}\right) C_{\left(e, w_{0}\right)}\left(k^{-\delta}\right) T_{w_{0}}
$$

with respect to the $\mathbb{C}$-basis $\left\{T_{w}\right\}_{w \in S_{N}}$ of $H_{0}$, we conclude that

$$
r_{\kappa}=\theta(\kappa)^{N} K\left(k^{\delta}\right)^{-1} \prod_{\beta \in R^{+}} c_{k}\left(k^{\langle\delta, \beta\rangle}\right) .
$$

Substituting the explicit expressions (2.5) and (5.16) of $c_{k}$ and $K$, respectively, we get the desired formula (7.7) for $r_{\kappa}$.

The following formula is an analog for the $Q_{\lambda}(\lambda \in \Lambda)$ of the evaluation formula for the self-dual symmetric Macdonald polynomials (see Subsection 7.4).

Corollary 7.9. Let $\lambda \in \Lambda$ and write $Q_{\lambda}(t)=\sum_{\alpha \in Q_{+}} K_{\alpha}(\lambda) t^{\lambda-\alpha}$ with $K_{\alpha}(\lambda) \in H_{0}$ (see Proposition 7.4). The leading coefficient $K_{0}(\lambda)$ is given by

$K_{0}(\lambda)=k^{\langle\delta, \lambda\rangle}\left(\prod_{1 \leq i<j \leq N} \prod_{m=0}^{\lambda_{i}-\lambda_{j}-1} \frac{1-q^{-m} k^{2(j-i)}}{1-q^{-m} k^{2(j-i+1)}}\right) k^{-\left(\begin{array}{c}N \\ 2\end{array}\right)} P\left(k^{2}\right) C_{\left(e, w_{0}\right)}\left(q^{\lambda} k^{-\delta}\right) T_{w_{0}}$ with

$$
P\left(k^{2}\right)=\prod_{1 \leq i<j \leq N} \frac{1-k^{2(j-i+1)}}{1-k^{2(j-i)}} .
$$

Proof. By (7.6) and Theorem 5.10 we have for $\lambda \in \Lambda$,

$$
K_{0}(\lambda)=r_{\kappa} k^{\langle\delta, \lambda\rangle} \theta(\kappa)^{-N} K\left(q^{w_{0}(\lambda)} k^{\delta}\right) C_{\left(e, w_{0}\right)}\left(q^{\lambda} k^{-\delta}\right) T_{w_{0}},
$$

with $K$ given by (5.16) and $r_{\kappa}$ given by (7.7). Substituting the explicit expressions for $K$ and $r_{\kappa}$ we get the desired expression.

The following consequence should be compared with the general expansion formula of $v_{+}=\sum_{w \in S_{N}} k^{\ell(w)} T_{w} \in H_{0}$ in terms of the $\xi_{w}(\gamma)\left(w \in S_{N}\right)$; see [37, Lemma $2.27(2)]$.

Corollary 7.10. The element $v_{+}=\sum_{w \in S_{N}} k^{\ell(w)} T_{w} \in H_{0}$ can be written as

$$
\begin{aligned}
& v_{+}=k^{-\left(\begin{array}{c}
N \\
2
\end{array}\right)} P\left(k^{2}\right) C_{\left(e, w_{0}\right)}\left(k^{-\delta}\right) T_{w_{0}} \\
& =\left(\prod_{1 \leq i<j \leq N} \frac{1}{1-k^{2(i-j)}}\right) \eta_{k^{-\delta}}\left(T_{w_{0}}\right) \xi_{w_{0}}\left(k^{-\delta}\right) .
\end{aligned}
$$

Proof. We have $v_{+}=Q_{0}=K_{0}(0)$, hence the previous corollary gives the first equality of (7.12). The second equality then follows from (7.9).

Applying the map $\chi_{+}$to the first line of (7.12) gives

$$
\sum_{w \in S_{N}} k^{2 \ell(w)}=P\left(k^{2}\right)
$$


with $P\left(k^{2}\right)$ given by (7.11), which is a well-known product formula for the Poincaré series of $S_{N}$; see [33, Cor. (2.5)].

7.4. Relation to symmetric self-dual Macdonald polynomials. In this subsection we collect various consequences of the previous subsections for the symmetric Laurent polynomials $\chi_{+}\left(Q_{\lambda}\right) \in \mathbb{C}[T]^{S_{N}}(\lambda \in \Lambda)$. We keep the generic conditions (7.1) on $k \in \mathbb{C}^{\times}$. We denote

$$
E_{\lambda}:=P\left(k^{2}\right)^{-1} \chi_{+}\left(Q_{\lambda}\right) \in \mathbb{C}[T]^{S_{N}}, \quad \lambda \in \Lambda .
$$

By Proposition, 7.4 we have

$$
E_{\lambda}(t)=\sum_{\alpha \in Q_{+}} K_{\alpha}^{+}(\lambda) t^{\lambda-\alpha}
$$

with $K_{\alpha}^{+}(\lambda)=P\left(k^{2}\right)^{-1} \chi_{+}\left(K_{\alpha}(\lambda)\right) \in \mathbb{C}$ all but finitely many zero, and with leading coefficient $K_{0}^{+}(\lambda) \neq 0$ by Corollary 7.9 and (7.1).

Theorem 7.11. The $E_{\lambda} \in \mathbb{C}[T]^{S_{N}}(\lambda \in \Lambda)$ are the symmetric self-dual Macdonald polynomials. In other words, the $E_{\lambda}$ are the unique symmetric regular functions on $T$ satisfying

$$
L_{p}^{x}\left(E_{\lambda}\right)=p\left(q^{-\lambda} k^{\delta}\right) E_{\lambda} \quad \forall p \in \mathbb{C}[T]^{S_{N}}
$$

and $E_{\lambda}\left(k^{\delta}\right)=1$ for all $\lambda \in \Lambda$.

Proof. By Proposition 6.22, $E_{\lambda} \in \mathbb{C}[T]^{S_{N}}$ satisfies (7.13). Since the $S_{N^{-}}$orbits $S_{N}\left(q^{-\lambda} k^{\delta}\right)(\lambda \in \Lambda)$ in $T$ are pairwise different by (7.1), the eigenvalue equations (7.13) uniquely characterize $E_{\lambda} \in \mathbb{C}[T]^{S_{N}}$ up to a nonzero constant multiple. Now

$$
E_{\lambda}\left(k^{\delta}\right)=P\left(k^{2}\right)^{-1} \chi_{+}\left(Q_{\lambda}\left(k^{\delta}\right)\right)=1
$$

by Lemma 7.5, which fixes the normalization of the solution $E_{\lambda} \in \mathbb{C}[T]^{S_{N}}$ of (7.13) uniquely.

The duality property of $Q_{\lambda}$ (see Proposition 7.6) immediately gives the wellknown duality property of the Macdonald polynomials.

Corollary 7.12. The Macdonald polynomials $E_{\lambda}(\lambda \in \Lambda)$ are self-dual, in the sense that

for all $\lambda, \mu \in \Lambda$.

$$
E_{\lambda}\left(q^{-\mu} k^{\delta}\right)=E_{\mu}\left(q^{-\lambda} k^{\delta}\right)
$$

Remark 7.13. The self-duality of (the suitably normalized) Macdonald polynomials was initially proved by Koornwinder using Pieri formulas in an unpublished manuscript (the argument is reproduced in [32, VI (6.6)]). Cherednik (9, Thm. 1.4.6] and 8 , Thm. 3.2]) reproduced the self-duality of the Macdonald polynomials using the anti-involution $*$ (see Theorem 2.8) on the double affine Hecke algebra.

We also immediately reobtain the well-known evaluation formula for the symmetric Macdonald polynomials; see [32, VI (6.11)] (the parameters $(n, q, t)$ in [32, Chpt. VI] correspond to $\left(N, q^{-1}, k^{2}\right)$ in our notations).

Corollary 7.14. For $\lambda \in \Lambda$ let $P_{\lambda}:=K_{0}^{+}(\lambda)^{-1} E_{\lambda} \in \mathbb{C}[T]^{S_{N}}$ be the monic symmetric Macdonald polynomial of degree $\lambda$. Then

$$
P_{\lambda}\left(k^{\delta}\right)=k^{-\langle\delta, \lambda\rangle} \prod_{1 \leq i<j \leq N} \prod_{m=0}^{\lambda_{i}-\lambda_{j}-1} \frac{1-q^{-m} k^{2(j-i+1)}}{1-q^{-m} k^{2(j-i)}} .
$$


Proof. By the previous theorem we have $P_{\lambda}\left(k^{\delta}\right)=K_{0}^{+}(\lambda)^{-1}$. Corollary 7.9 gives

$$
K_{0}^{+}(\lambda)=k^{\langle\delta, \lambda\rangle} \prod_{1 \leq i<j \leq N} \prod_{m=0}^{\lambda_{i}-\lambda_{j}-1} \frac{1-q^{-m} k^{2(j-i)}}{1-q^{-m} k^{2(j-i+1)}},
$$

which implies the desired result.

\section{Appendix on holonomic Systems of $q$-Difference EQUations}

In the appendix we detail the construction of power series solutions of holonomic systems of $q$-difference equations. Special cases have been investigated in, e.g., 2], [20] and [14, §12]. Many arguments go back to classical works [1, [3], 4, 48] on ordinary linear $q$-difference equations.

We begin with the construction of formal asymptotic solutions to holonomic systems of $q$-difference equations. Let $\mathbb{C}[[z]]=\mathbb{C}\left[\left[z_{1}, \ldots, z_{M}\right]\right]$ denote the ring of formal power series in $M$ indeterminates $z_{1}, \ldots, z_{M}$ over the complex numbers. Let $V$ be a finite-dimensional complex vector space and let

$$
A_{i} \in \mathbb{C}[[z]] \otimes \operatorname{End}(V)
$$

for $i=1, \ldots, M$. Since $\mathbb{C}[[z]] \otimes \operatorname{End}(V)$ is isomorphic to $\operatorname{End}_{\mathbb{C}[[z]]}(\mathbb{C}[[z]] \otimes V)$ as $\mathbb{C}[[z]]$-module, we can view the $A_{i}$ as $\mathbb{C}[[z]]$-linear endomorphisms of $\mathbb{C}[[z]] \otimes V$. Fix $0<q_{i}<1$ for $1 \leq i \leq M$. Define the $q_{i}$-dilation operators

$$
\mathcal{T}_{i}: \mathbb{C}[[z]] \rightarrow \mathbb{C}[[z]]
$$

for $i=1, \ldots, M$ as the complex linear maps

$$
\mathcal{T}_{i}\left(\sum_{\mathbf{m}} d_{\mathbf{m}} z^{\mathbf{m}}\right):=\sum_{\mathbf{m}} q_{i}^{m_{i}} d_{\mathbf{m}} z^{\mathbf{m}} \quad\left(d_{\mathbf{m}} \in \mathbb{C}\right),
$$

where we use multi-index notation $z^{\mathbf{m}}=z_{1}^{m_{1}} \cdots z_{M}^{m_{M}}$ for $\mathbf{m}=\left(m_{1}, \ldots, m_{M}\right)$ with $m_{j} \in \mathbb{Z}_{\geq 0}$. We also view $\mathcal{T}_{i}$ as operators on $\mathbb{C}[[z]] \otimes V$ and on $\mathbb{C}[[z]] \otimes \operatorname{End}(V)$. Consider the system of first-order linear $q$-difference equations

$$
A_{i} \mathcal{T}_{i} f=f, \quad(i=1, \ldots, M)
$$

for $f \in \mathbb{C}[[z]] \otimes V$.

For $f \in \mathbb{C}[[z]] \otimes V$ and $A \in \mathbb{C}[[z]] \otimes \operatorname{End}(V)$, we introduce the notations

$$
\begin{aligned}
& f^{(m)}:=\left.f\right|_{z_{m+1}=\ldots=z_{M}=0} \in \mathbb{C}\left[\left[z_{1}, \ldots, z_{m}\right]\right] \otimes V \\
& A^{(m)}:=\left.A\right|_{z_{m+1}=\ldots=z_{M}=0} \in \mathbb{C}\left[\left[z_{1}, \ldots, z_{m}\right]\right] \otimes \operatorname{End}(V)
\end{aligned}
$$

for $0 \leq m \leq M$, with the convention that $f^{(M)}=f$ and $A^{(M)}=A$. We make the following assumptions on the system of $q$-difference equations (8.1):

(a) The system (8.1) is holonomic, that is

$$
A_{i} \mathcal{T}_{i}\left(A_{j}\right)=A_{j} \mathcal{T}_{j}\left(A_{i}\right)
$$

for all $1 \leq i, j \leq M$. Note that the holonomy implies that the leading coefficients $A_{i}^{(0)} \in \operatorname{End}(V)$ of $A_{i}$ mutually commute, i.e.,

$$
\left[A_{i}^{(0)}, A_{j}^{(0)}\right]=0
$$

for all $1 \leq i, j \leq M$. 
(b) The complex linear endomorphisms $A_{1}^{(0)}, \ldots, A_{M}^{(0)}$ of $V$ are semisimple. Combined with (a) we thus have

$$
V=\bigoplus_{\gamma \in S} V[\gamma]
$$

with $V[\gamma]:=\left\{v \in V \mid A_{i}^{(0)} v=\gamma_{i} v \forall i\right\}\left(\gamma \in \mathbb{C}^{M}\right)$ and $S:=\left\{\gamma \in \mathbb{C}^{M} \mid V[\gamma] \neq\{0\}\right\}$.

(c) $\left(1^{M}\right):=(1, \ldots, 1) \in \mathbb{C}^{M}$ belongs to $S$.

(d) $\gamma_{k} \notin q_{k}^{-\mathbb{N}}$ for all $\gamma \in S$ and $1 \leq k \leq M$.

Proposition 8.1. Fix $v \in V\left[\left(1^{M}\right)\right]$. Consider the system (8.1) of q-difference equations and suppose that (a)-(d) are satisfied. Then there exists a unique solution $\Phi_{v} \in \mathbb{C}[[z]] \otimes V$ of (8.1) such that

$$
\Phi_{v}^{(0)}=v .
$$

Proof. The proposition is a consequence of the following lemma.

Lemma 8.2. Let $0 \leq m<M$. Suppose one has a solution

$$
f_{m} \in \mathbb{C}\left[\left[z_{1}, \ldots, z_{m}\right]\right] \otimes V
$$

of the system of equations

$$
\begin{aligned}
A_{r}^{(m)} \mathcal{T}_{r} f_{m} & =f_{m}, & & 1 \leq r \leq m, \\
A_{s}^{(m)} f_{m} & =f_{m}, & & m<s \leq M .
\end{aligned}
$$

Then there exists a unique

$$
f_{m+1}=\sum_{n \geq 0} f_{m ; n} z_{m+1}^{n} \in \mathbb{C}\left[\left[z_{1}, \ldots, z_{m+1}\right]\right] \otimes V
$$

with $f_{m ; n} \in \mathbb{C}\left[\left[z_{1}, \ldots, z_{m}\right]\right] \otimes V$ and $f_{m ; 0}=f_{m}$ satisfying (8.3) with the role of $m$ replaced by $m+1$ :

$$
\begin{aligned}
A_{r}^{(m+1)} \mathcal{T}_{r} f_{m+1} & =f_{m+1}, & & 1 \leq r \leq m+1, \\
A_{s}^{(m+1)} f_{m+1} & =f_{m+1}, & & m+1<s \leq M .
\end{aligned}
$$

The proposition follows directly from the lemma as follows. Note that $f_{0}:=v \in$ $V\left[\left(1^{M}\right)\right]$ is a solution of (8.3) for $m=0$. The formal $V$-valued series $f_{M} \in \mathbb{C}[[z]] \otimes V$, obtained by repeated application of the lemma starting from $f_{0}=v$, gives a formal $V$-valued series solution of (8.1) satisfying $f_{M}^{(0)}=v$. For uniqueness, assume that $f \in \mathbb{C}[[z]] \otimes V$ is another formal $V$-valued series satisfying $f^{(0)}=v$ and solving (8.1). We have $f^{(0)}=v=f_{0}$ and $f^{(m)}$ solves (8.3) for all $0 \leq m<M$. Hence, by the uniqueness part of the lemma, $f=f^{(M)}=f_{M}$.

We now proceed to prove the lemma. We assume that we have a formal power series solution $f_{m} \in \mathbb{C}\left[\left[z_{1}, \ldots, z_{m}\right]\right] \otimes V$ of (8.3) for some $0 \leq m<M$. We write

$$
A_{r}^{(m+1)}=\sum_{n \geq 0} A_{r ; n}^{(m)} z_{m+1}^{n}
$$

where $A_{r ; n}^{(m)} \in \mathbb{C}\left[\left[z_{1}, \ldots, z_{m}\right]\right] \otimes \operatorname{End}(V)$ and $A_{r ; 0}^{(m)}=A_{r}^{(m)}$. By a direct computation one verifies that

$$
f_{m+1}=\sum_{n \geq 0} f_{m ; n} z_{m+1}^{n} \in \mathbb{C}\left[\left[z_{1}, \ldots, z_{m+1}\right]\right] \otimes V
$$


with $f_{m ; n} \in \mathbb{C}\left[\left[z_{1}, \ldots, z_{m}\right]\right] \otimes V$ and $f_{m ; 0}=f_{m}$ satisfies the $q$-difference equation

$$
A_{m+1}^{(m+1)} \mathcal{T}_{m+1} f_{m+1}=f_{m+1}
$$

if and only if

$$
\left(1-q_{m+1}^{n} A_{m+1}^{(m)}\right) f_{m ; n}=\sum_{l=1}^{n} q_{m+1}^{n-l} A_{m+1 ; l}^{(m)} f_{m ; n-l}
$$

for all $n \in \mathbb{Z}_{\geq 0}$. The recurrence relations (8.6) admit a unique solution $\left(f_{m ; n}\right)_{n \in \mathbb{Z}_{\geq 0}}$ with $f_{m ; n} \in \mathbb{C}\left[\left[z_{1}, \ldots, z_{m}\right]\right] \otimes V$ and with initial condition $f_{m ; 0}=f_{m}$. Indeed, note that (8.6) is valid for $n=0$ since $f_{m ; 0}=f_{m}$ satisfies (8.3). For $n \geq 1$, we have

$$
\operatorname{det}\left(1-q_{m+1}^{n} A_{m+1}^{(m)}\right) \in \mathbb{C}\left[\left[z_{1}, \ldots, z_{m}\right]\right]^{\times},
$$

since

$\left.\operatorname{det}\left(1-q_{m+1}^{n} A_{m+1}^{(m)}\right)\right|_{z_{1}=\ldots=z_{m}=0}=\operatorname{det}\left(1-q_{m+1}^{n} A_{m+1}^{(0)}\right)=\prod_{\gamma \in S}\left(1-q_{m+1}^{n} \gamma_{m+1}^{\operatorname{dim}(V[\gamma])}\right) \neq 0$

by assumption (d). Cramer's rule then implies that (8.6) admits a unique solution $\left(f_{m ; n}\right)_{n \in \mathbb{Z}_{\geq 0}}$ with $f_{m ; 0}=f_{m}$.

We conclude that there exists a unique

$$
f_{m+1}=\sum_{n \geq 0} f_{m ; n} z_{m+1}^{n}
$$

with $f_{m ; n} \in \mathbb{C}\left[\left[z_{1}, \ldots, z_{m}\right]\right] \otimes V$ and $f_{m ; 0}=f_{m}$ satisfying the $q$-difference equation

$$
A_{m+1}^{(m+1)} \mathcal{T}_{m+1} f_{m+1}=f_{m+1} \text {. }
$$

It remains to show that $f_{m+1}$ also satisfies (8.4) for $r=1, \ldots, m$ and for $s=$ $m+2, \ldots, M$.

Fix $1 \leq r \leq m$ and write $g_{r}:=A_{r}^{(m+1)} \mathcal{T}_{r} f_{m+1}$. Its expansion in powers of $z_{m+1}$ is written as

$$
g_{r}=\sum_{n \geq 0} g_{r ; n} z_{m+1}^{n}
$$

with $g_{r ; n} \in \mathbb{C}\left[\left[z_{1}, \ldots, z_{m}\right]\right] \otimes V$ and $g_{r ; 0}=A_{r}^{(m)} \mathcal{T}_{r} f_{m}=f_{m}$, where the last equality follows from the fact that $f_{m}$ is assumed to satisfy (8.3). Furthermore, using the holonomy (8.2) and the $q$-difference equation (8.7) in $z_{m+1}$ satisfied by $f_{m+1}$, we have

$$
\begin{aligned}
A_{m+1}^{(m+1)} \mathcal{T}_{m+1} g_{r} & =A_{m+1}^{(m+1)} \mathcal{T}_{m+1}\left(A_{r}^{(m+1)}\right) \mathcal{T}_{r} \mathcal{T}_{m+1} f_{m+1} \\
& =A_{r}^{(m+1)} \mathcal{T}_{r}\left(A_{m+1}^{(m+1)}\right) \mathcal{T}_{r} \mathcal{T}_{m+1} f_{m+1} \\
& =A_{r}^{(m+1)} \mathcal{T}_{r}\left(A_{m+1}^{(m+1)} \mathcal{T}_{m+1} f_{m+1}\right) \\
& =A_{r}^{(m+1)} \mathcal{T}_{r} f_{m+1}=g_{r} .
\end{aligned}
$$

We conclude that $g_{r}$ satisfies the characterizing properties of $f_{m+1}$. Hence $g_{r}=$ $f_{m+1}$, i.e.,

$$
A_{r}^{(m+1)} \mathcal{T}_{r} f_{m+1}=f_{m+1} .
$$

Fix $m+1<s \leq M$ and write $g_{s}:=A_{s}^{(m+1)} f_{m+1}$. By a similar argument as used in the previous paragraph, we now show that $g_{s}=f_{m+1}$. We write

$$
g_{s}=\sum_{n \geq 0} g_{s ; n} z_{m+1}^{n}
$$


with $g_{s ; n} \in \mathbb{C}\left[\left[z_{1}, \ldots, z_{m}\right]\right] \otimes V$ and $g_{s ; 0}=A_{s}^{(m)} f_{m}=f_{m}$, where the last equality follows by the assumption that $f_{m}$ satisfies (8.3). Using the holonomy (8.2), the $q$-difference equation (8.7), and the obvious fact that $\mathcal{T}_{s}\left(A_{m+1}^{(m+1)}\right)=A_{m+1}^{(m+1)}$ since $s>m+1$, we have

$$
\begin{aligned}
A_{m+1}^{(m+1)} \mathcal{T}_{m+1} g_{s} & =A_{m+1}^{(m+1)} \mathcal{T}_{m+1}\left(A_{s}^{(m+1)}\right) \mathcal{T}_{m+1} f_{m+1} \\
& =A_{s}^{(m+1)} \mathcal{T}_{s}\left(A_{m+1}^{(m+1)}\right) \mathcal{T}_{m+1} f_{m+1} \\
& =A_{s}^{(m+1)} A_{m+1}^{(m+1)} \mathcal{T}_{m+1} f_{m+1} \\
& =A_{s}^{(m+1)} f_{m+1}=g_{s}
\end{aligned}
$$

We conclude that $g_{s}$ satisfies the characterizing properties of $f_{m+1}$. Hence $g_{s}=$ $f_{m+1}$, i.e.

$$
A_{s}^{(m+1)} f_{m+1}=f_{m+1}
$$

This completes the proof of Lemma 8.2 and hence the proof of Proposition 8.1.

We investigate the analytical properties of the solution $\Phi_{v}$ when the $q$-connection matrices $A_{i}(1 \leq i \leq M)$ satisfy, besides the conditions (a)-(d), the following analyticity condition:

(e) For some $\epsilon>0$ the formal $\operatorname{End}(V)$-valued series $A_{i} \in \mathbb{C}[[z]] \otimes \operatorname{End}(V)$ $(1 \leq i \leq M)$ converges normally on compacta of the open polydisc $D_{\epsilon}^{M}:=\{z \in$ $\left.\mathbb{C}^{M}|| z_{i} \mid<\epsilon \forall i\right\}$.

In other words, if we expand $A_{i}$ along a basis of $\operatorname{End}(V)$, condition (e) requires its coefficients in $\mathbb{C}[[z]]$ to converge normally on compacta of $D_{\epsilon}^{M}$.

Proposition 8.3. Suppose that the q-connection matrices $A_{i} \in \mathbb{C}[[z]] \otimes \operatorname{End}(V)$ $(1 \leq i \leq M)$ satisfy $(\mathbf{a})-(\mathbf{e})$. Let $v \in V\left[\left(1^{M}\right)\right]$. There exists an $\epsilon>0$ such that the formal $V$-valued series $\Phi_{v} \in \mathbb{C}[[z]] \otimes V$ converges normally on compacta of $D_{\epsilon}^{M}$.

Proof. For ease of notation, we will write $\Phi$ instead of $\Phi_{v}$. By induction on $m=$ $0, \ldots, M$ we prove that there exists $\epsilon>0$ such that $\Phi^{(m)} \in \mathbb{C}\left[\left[z_{1}, \ldots, z_{m}\right]\right] \otimes V$ converges normally on compacta of $D_{\epsilon}^{m}$.

For $m=0$, there is nothing to prove. Fix $0 \leq m<M$ and suppose $\Phi^{(m)}$ converges normally on compacta of $D_{\delta}^{m}$ for some $\delta>0$. Write

$$
\Phi^{(m+1)}=\sum_{n \geq 0} \Phi_{m ; n} z_{m+1}^{n}
$$

with $\Phi_{m ; n} \in \mathbb{C}\left[\left[z_{1}, \ldots, z_{m}\right]\right] \otimes V$ and $\Phi_{m ; 0}=\Phi^{(m)}$. Recall from the proof of Lemma 8.2 that the formal $V$-valued power series $\Phi_{m ; n}(n \geq 1)$ are unique characterized by the recurrence relations

$$
\Phi_{m ; n}=\sum_{l=1}^{n} q_{m+1}^{n-l}\left(1-q_{m+1}^{n} A_{m+1}^{(m)}\right)^{-1} A_{m+1 ; l}^{(m)} \Phi_{m ; n-l}
$$

for all $n \geq 1$. We use this recurrence formula to find bounds for $\Phi_{m ; n}$ in a neighborhood of $0 \in \mathbb{C}^{m}$.

Turn the finite-dimensional complex vector space $V$ into an inner product space, with corresponding norm denoted by $\|\cdot\|$. We also write $\|\cdot\|$ for the operator norm of the associated finite-dimensional normed space $\operatorname{End}(V)$. We continue the proof of the proposition with two technical sublemmas. First we find a proper uniform bound for $A_{m+1 ; l}^{(m)}$ for all $l$ (see (8.8)). 
Lemma 8.4. There exists $\epsilon>0$ and $M>0$ such that $\left\|A_{m+1 ; l}^{(m)}\right\| \leq M \epsilon^{-l}$ on $\bar{D}_{\epsilon}^{m}$ for all $l \geq 0$.

Proof. By (e) there exists an $\epsilon>0$ such that $A_{m+1}^{(m+1)} \in \mathbb{C}\left[\left[z_{1}, \ldots, z_{m+1}\right]\right] \otimes \operatorname{End}(V)$ converges normally on compacta of the polydisc $D_{2 \epsilon}^{m+1}$. Consequently, for $\epsilon<\epsilon^{\prime}<$ $2 \epsilon$ we have that $\left\|A_{m+1}^{(m+1)}\right\|$ is uniformly bounded on the polydisc $D_{\epsilon^{\prime}}^{m+1}$, say by $M>0$. In particular, we get

$$
\left\|\left.\left(\partial_{z_{m+1}}^{l} A_{m+1}^{(m+1)}\right)\left(z_{1}, \ldots, z_{m+1}\right)\right|_{z_{m+1}=0}\right\| \leq M \epsilon^{-l} l !
$$

for all $\left(z_{1}, \ldots, z_{m}\right) \in \bar{D}_{\epsilon}^{m}$ and for all $l \geq 0$ (see, e.g., 223] Theorem 2.2.7). This proves the lemma in view of the definition (8.5) of $A_{m+1 ; l}^{(m)}$.

Lemma 8.5. There exists an $\epsilon>0$ such that $\Phi_{m ; n} \in \mathbb{C}\left[\left[z_{1}, \ldots, z_{m}\right]\right] \otimes V$ converges normally on compacta of $D_{\epsilon}^{m}$ for all $n \geq 0$. Furthermore, there exists a constant $C>0$ (independent of $n$ ) such that

$$
\left\|\Phi_{m ; n}\right\| \leq \frac{C}{1+C}\left(\frac{1+C}{q_{m+1} \epsilon}\right)^{n}\left\|\Phi^{(m)}\right\|
$$

on $D_{\epsilon}^{m}$ for all $n \geq 1$.

Proof. In the proof of this lemma, we write $q$ instead of $q_{m+1}$. By assumption, $\Phi_{m ; 0}=\Phi^{(m)}$ converges normally on compacta of $D_{\epsilon}^{m}$ if $0<\epsilon<\delta$. We now use the recurrence relation (8.8) to obtain the desired results for $\Phi_{m ; n}$ with $n \geq 1$.

By the proof of Lemma 8.2 and since $0<q<1$, there exists some $\epsilon>0$ (independent of $n \geq 1$ ) such that $\operatorname{det}\left(1-q^{n} A_{m+1}^{(m)}\right)^{-1}$ is analytic on $D_{\epsilon}^{m}$ for all $n \geq 1$ and such that $\left|\operatorname{det}\left(1-q^{n} A_{m+1}^{(m)}\right)^{-1}\right|$ is bounded on the closure $\bar{D}_{\epsilon}^{m}$ of $D_{\epsilon}^{m}$, with bound independent of $n \geq 1$. For such $\epsilon$, it follows from (8.8) that $\Phi_{m ; n}$ converges normally on compacta of $D_{\epsilon}^{m}$ for all $n \geq 1$. Furthermore, by (e), $0<q<1$, and Cramer's rule, it implies that for $\epsilon>0$ small enough,

$$
\left\|\left(1-q^{n} A_{m+1}^{(m)}\right)^{-1}\right\| \leq C^{\prime}
$$

on $\bar{D}_{\epsilon}^{m}$ for all $n \geq 1$, with $C^{\prime}>0$ also independent of $n$. By (8.8), $0<q<1$ and the previous lemma, we thus obtain for $\epsilon>0$ small enough,

$$
\left\|\Phi_{m ; n}\right\| \leq C^{\prime} \sum_{l=1}^{n} q^{n-l}\left\|A_{m+1 ; l}^{(m)}\right\|\left\|\Phi_{m ; n-l}\right\| \leq C \sum_{l=1}^{n}\left(\frac{1}{q \epsilon}\right)^{l}\left\|\Phi_{m ; n-l}\right\|
$$

on $\bar{D}_{\epsilon}^{m}$ for all $n \geq 1$ with the constant $C=C^{\prime} M>0$ (independent of $n$ ).

Now, we have the following claim (cf. [14 §10.6): the recurrence relation

$$
g_{n}=C \sum_{l=1}^{n}\left(\frac{1}{q \epsilon}\right)^{l} g_{n-l}, \quad(n>0)
$$

with $g_{0} \in \mathbb{R}$ fixed is uniquely solved by

$$
g_{n}=\frac{C}{C+1}\left(\frac{1+C}{q \epsilon}\right)^{n} g_{0}
$$


for $n \geq 1$. Being obvious for $n=1$, the claim follows using induction for $n>1$ by

$$
\begin{aligned}
g_{n} & =C\left(\frac{1}{q \epsilon}\right)^{n} g_{0}+C \sum_{l=1}^{n-1}\left(\frac{1}{q \epsilon}\right)^{l} g_{n-l} \\
& =C\left(\frac{1}{q \epsilon}\right)^{n} g_{0}+\frac{C^{2}}{C+1} \sum_{l=1}^{n-1}\left(\frac{1}{q \epsilon}\right)^{l}\left(\frac{(1+C)}{q \epsilon}\right)^{n-l} g_{0} \\
& =C\left(\frac{1}{q \epsilon}\right)^{n} g_{0}\left(1+C \sum_{l=0}^{n-2}(1+C)^{l}\right) \\
& =C\left(\frac{1}{q \epsilon}\right)^{n} g_{0}\left(1+C\left(\frac{(1+C)^{n-1}-1}{1+C-1}\right)\right) \\
& =\frac{C}{C+1}\left(\frac{1+C}{q \epsilon}\right)^{n} g_{0} .
\end{aligned}
$$

Combined with (8.9), the lemma now follows immediately.

To conclude the proof of the proposition, note that the previous lemma shows that

$$
\Phi^{(m+1)}=\sum_{n \geq 0} \Phi_{m ; n} z_{m+1}^{n} \in \mathbb{C}\left[\left[z_{1}, \ldots, z_{m+1}\right]\right] \otimes \operatorname{End}(V)
$$

converges normally on compacta of $D_{\epsilon^{\prime}}^{m+1}$ if we take $\epsilon^{\prime}>0$ sufficiently small. This concludes the proof of the induction step.

We interpret the $q$-dilation operators $\mathcal{T}_{i}$ as automorphisms of $\mathcal{M}\left(\mathbb{C}^{M}\right)$ by

$$
\left(\mathcal{T}_{i} f\right)(z)=f\left(z_{1}, \ldots, z_{i-1}, q_{i} z_{i}, z_{i+1}, \ldots, z_{M}\right) .
$$

Theorem 8.6. Suppose $A_{i} \in \mathcal{M}\left(\mathbb{C}^{M}\right) \otimes \operatorname{End}(V)(1 \leq i \leq M)$ satisfy the holonomy conditions (8.2) as meromorphic End $(V)$-valued functions on $\mathbb{C}^{M}$. Suppose that the $A_{i}$ are analytic at $0 \in \mathbb{C}^{M}$ and that their power series expansions at $0 \in \mathbb{C}^{M}$ satisfy the conditions $(\mathbf{b})-(\mathbf{d})$.

Let $v \in V\left[\left(1^{M}\right)\right]$. There exists a unique $\Phi_{v} \in \mathcal{M}\left(\mathbb{C}^{M}\right) \otimes V$ solving the holonomic system (8.1) of q-difference equations and coinciding, in a small neighborhood of $0 \in \mathbb{C}^{M}$, with the converging $V$-valued power series solution $\Phi_{v}$ from Proposition 8.3 .

Proof. Since the $A_{i}$ are assumed to be analytic at $0 \in \mathbb{C}^{M}$, their power series expansions at $0 \in \mathbb{C}^{M}$ are converging normally on compacta of some open polydisc $D_{\epsilon}^{M}(\epsilon>0)$. Hence, condition (e) is automatically satisfied.

Let $\Phi_{v} \in \mathbb{C}[[z]] \otimes V$ be the power series solution from Proposition 8.3 and let $\epsilon>0$ such that $\Phi_{v}$ converges normally on compacta of $D_{\epsilon}^{M}$. Let $z^{\prime} \in \mathbb{C}^{M}$ and $U \subset \mathbb{C}^{M}$ some open locally compact neighborhood of $z^{\prime}$. Since $0<q_{i}<1(1 \leq i \leq M)$, there exists a $\lambda \in \mathbb{Z}_{\geq 0}^{M}$ such that $q^{\lambda} U \subset D_{\epsilon}^{M}$, where $q^{\lambda} z=\left(q_{1}^{\lambda_{1}} z_{1}, \ldots, q_{M}^{\lambda_{M}} z_{M}\right)$. Define $\Phi_{v}$ as $V$-valued meromorphic function on $z \in U$ by

$$
\Phi_{v}(z)=A_{\lambda}(z) \Phi_{v}\left(q^{\lambda} z\right),
$$

where $A_{\lambda} \in \mathcal{M}\left(\mathbb{C}^{M}\right) \otimes \operatorname{End}(V)$ is defined inductively by

$$
A_{\lambda+\mu}(z)=A_{\lambda}(z) A_{\mu}\left(q^{\lambda} z\right), \quad \forall \lambda, \mu \in \mathbb{Z}_{\geq 0}^{M},
$$

and $A_{\epsilon_{i}}=A_{i}(1 \leq i \leq M)$, where the $\epsilon_{i}(1 \leq i \leq M)$ are the standard generators of the additive monoid $\mathbb{Z}_{\geq 0}^{M}$. Of course, the definition of $A_{\lambda}(z)$ makes sense by the 
holonomy conditions for the $A_{i}$. Furthermore, 8.10) together with the holonomy conditions for the $A_{i}$ show that the power series solution $\Phi_{v}$ of (8.2) has a unique extension to a meromorphic $V$-valued solution on $\mathbb{C}^{M}$ of (8.2).

\section{REFERENCES}

[1] C.R. Adams, On the linear ordinary q-difference equation, Ann. Math. 30 (1929), 195-205.

[2] K. Aomoto, A note on holonomic q-difference systems, in: "Algebraic analysis", Vol. I, 25-28, Academic Press, Boston, MA, 1988.

[3] G.D. Birkhoff, The generalized Riemann problem for linear differential equations and the allied problems for linear difference and q-difference equations, Proc. Amer. Acad. 49 (1913), $521-568$.

[4] R.D. Carmichael, The general theory of linear q-difference equations, Amer. J. Math. 34 (1912), 147-168.

[5] O. Chalykh, Macdonald polynomials and algebraic integrability, Adv. Math. 166 (2002), no. $2,193-259$.

[6] I. Cherednik, Double affine Hecke algebras, Knizhnik-Zamolodchikov equations, and Macdonald's operators, Int. Math. Res. Not. 1992, No. 9, 171-180.

[7] I. Cherednik, Induced representations of double affine Hecke algebras and applications, Math. Res. Lett. 1 (1994), 319-337.

[8] I. Cherednik, Macdonald's evaluation conjectures and difference Fourier transform, Invent. Math. 122 (1995), 119-145.

[9] I. Cherednik, Double affine Hecke algebras, Cambridge University Press, London Math. Soc. Lecture Note Series 319, 2005.

[10] I. Cherednik, Whittaker limits of difference spherical functions, Int. Math. Res. Not. 2009, No. 20, 3793-842.

[11] P. Di Francesco, P. Zinn-Justin, Quantum Knizhnik-Zamolodchikov equation, generalized Razumov-Stroganov sum rules and extended Joseph polynomials, J. Phys. A 38 (2005), L815L822.

[12] J.J. Duistermaat, F.A. Grünbaum, Differential equations in the spectral parameter, Comm. Math. Phys. 103 (1986), no. 2, 177-240.

[13] P.I. Etingof, Difference equations with elliptic coefficients and quantum affine algebras (1993), arXiv:hep-th/9312057.

[14] P.I. Etingof, I.B. Frenkel, A.A. Kirillov Jr., Lectures on Representation Theory and KnizhnikZamolodchikov Equations, Math. Surveys and Monographs, Vol. 58, Amer. Math. Soc., 1998.

[15] P. Etingof, A.A. Kirillov Jr., Macdonald's polynomials and representations of quantum groups, Math. Res. Lett. 1 (1994), no. 3, 279-296.

[16] P. Etingof, A.A. Kirillov Jr., Representation-theoretic proof of the inner product and symmetry identities for Macdonald's polynomials, Compositio Math. 102 (1996), no. 2, 179-202.

[17] P. Etingof, K. Styrkas, Algebraic integrability of Macdonald polynomials and representations of quantum groups, Compositio Math. 114 (1998), 125-152.

[18] P. Etingof, A. Varchenko, Dynamical Weyl groups and applications, Adv. Math. 167 (2002), no. $1,74-127$.

[19] G. Felder, Ya. Markov, V. Tarasov, A. Varchenko, Differential equations compatible with KZ equations, Math. Phys. Anal. Geom. 3 (2000), 139-177.

[20] I.B. Frenkel, N.Yu. Reshetikhin, Quantum affine algebras and holonomic difference equations, Comm. Math. Phys. 146 (1992), no. 1, 1-60.

[21] F.A. Grünbaum, L. Haine, Some functions that generalize the Askey-Wilson polynomials, Comm. Math. Phys. 184 (1997), no. 1, 173-202.

[22] J. Harnad, Quantum Isomonodromic deformations and the Knizhnik-Zamolodchikov equations. In: "Symmetries and integrability of difference equations" (Estérel, PQ, 1994), 155161, CRM Proc. Lecture Notes, 9, Amser. Math. Soc., Providence, RI, 1996.

[23] L. Hörmander, An Introduction to Complex Analysis in Several Variables. Second revised edition. North-Holland Mathematical Library, Vol. 7. North-Holland Publishing Co., Amsterdam-London; American Elsevier Publishing Co., Inc., New York, 1973.

[24] M. Jimbo, T. Miwa, Algebraic analysis of solvable lattice models. CBMS Regional Cinference Series in Mathematics 85. Published for the Conference Board of the Mathematical Sciences, Washington, DC; by the American Mathematical Society, Providence, RI, 1995. 
[25] M. Kasatani, Y. Takeyama, The quantum Knizhnik-Zamolodchikov equation and nonsymmetric Macdonald polynomials, Funkcialaj Ekvacioj 50 (2007), 491-509.

[26] S. Kato, R-matric arising from affine Hecke algebras and its applications to Macdonald's difference operators, Comm. Math. Phys. 165 (1994), no. 3, 533-553.

[27] A. Kazarnovski-Krol, Matrix elements of vertes operators of the deformed $\mathrm{WA}_{n}$-algebra and the Harish-Chandra solutions to Macdonald's difference equations, Selecta Math. (N.S.) 5 (1999), no. 2, 257-301.

[28] A.N. Kirillov, M. Noumi, Affine Hecke algebras and raising operators for Macdonald polynomials, Duke Math. J. 93 (1998), no. 1, 1-39.

[29] V.P. Leksin, Isomonodromic and bispectral properties of the generalized KnizhnikZamolodchikov equations, J. Math. Sci. (N.Y.) 129 (2005), no. 1, 3635-3641.

[30] G. Lusztig, Affine Hecke Algebras and their Graded Version, J. Amer. Math. Soc. 2 (1989), no. 3, 599-635.

[31] G. Letzter, J.V. Stokman, Macdonald difference operators and Harish-Chandra series, Proc. Lond. Math. Soc. 97 (2008), no. 1, 60-96.

[32] I.G. Macdonald, Symmetric functions and Hall polynomials. Second edition, Oxford Math. Monographs, Oxford Science Publications, The Clarendon Press, Oxford University Press, New York, 1995.

[33] I.G. Macdonald, The Poincaré series of a Coxeter group, Math. Ann. 199 (1972), 161-174.

[34] I.G. Macdonald, Affine Hecke algebras and orthogonal polynomials, Cambridge Tracts in Mathematics 157, Cambridge University Press, Cambridge, 2003.

[35] A. Matsuo, Integrable connections related to zonal spherical functions, Invent. Math. 110 (1992), 96-121.

[36] K. Mimachi, A solution to quantum Knizhnik-Zamolodchikov equations and its applications to eigenvalue problems of the Macdonald type, Duke Math. J. 85 (1996), no. 3, 635-658.

[37] E.M. Opdam, A generating function for the trace of the Iwahori-Hecke algebra, in: Studies in memory of Issai Schur (Chevaleret/Rehovot, 2000), 301-323, Progr. Math., 210, Birkhäuser Boston, Boston, MA, 2003.

[38] N. Reshetikhin, The Knizhnik-Zamolodchikov system as a deformation of the isomonodromy problem, Lett. Math. Phys. 26 (1992), 167-177.

[39] S.N.M. Ruijsenaars, Complete integrability of relativistic Calogero-Moser systems and elliptic function identities, Comm. Math. Phys. 110 (1987), 191-213.

[40] S.N.M. Ruijsenaars, Factorized weight functions vs. factorized scattering, Comm. Math. Phys. 228 (2002), no. 3, 467-494.

[41] J. Sauloy, Systemes aux q-différences singuliers réguliers: classification, matrice de connexion et monodromie, Ann. Inst. Fourier (Grenoble) 50 (2000), no. 4, 1021-1071.

[42] F. Smirnov, General formula for soliton form factors in sine-Gordon model, J. Phys. A 19 (1986), no. 10, L575-L578.

[43] J.-P. Serre, Local Fields, Graduate Texts in Mathematics 67, Springer-Verlag, New YorkBerlin, 1979.

[44] V. Tarasov, Duality for Knizhnik-Zamolodchikov and dynamical equations, and hypergeometric integrals, in "Infinite dimensional algebras and quantum integrable systems", 235-263, Prog. Math., 237, Birkhäuser, Basel, 2005.

[45] V. Tarasov, A. Varchenko, Difference equations compatible with trigonometric KZ differential equations, Int. Math. Res. Not. 2000, no. 15, 801-829.

[46] V. Tarasov, A. Varchenko, Duality for Knizhnik-Zamolodchikov and dynamical equations. The 2000 Twente conference on Lie groups (Enschede) Acta Appl. Math. 73 (2002), no. 1-2, $141-154$

[47] V. Toledano Laredo, A Kohno-Drinfeld theorem for quantum Weyl groups, Duke Math. J. 112 (2002), no. 3, 421-451.

[48] W.J. Trjitzinsky, Analytic theory of linear q-difference equations, Acta Math. 61 (1933), $1-38$.

KdV Institute for Mathematics, University of Amsterdam, Science Park 904, 1098 XH Amsterdam, The Netherlands.

E-mail address: m.vanmeer@uva.nl, j.v.stokman@uva.nl 